\title{
Enumerative properties of restricted words and compositions
}

\author{
Andrew MacFie \\ A thesis submitted to the Faculty of Graduate and Postdoctoral Affairs in partial \\ fulfillment of the requirements for the degree of \\ Doctor of Philosophy \\ in \\ Mathematics \\ School of Mathematics and Statistics \\ Ottawa-Carleton Institute for Mathematics and Statistics \\ Carleton University \\ Ottawa, Ontario \\ C2018 Andrew MacFie
}


It is not knowledge, but the act of learning, not possession but the act of getting there, which grants the greatest enjoyment.

-Carl Friedrich Gauss, 1808 


\section{Abstract}

Words and integer compositions are fundamental combinatorial objects. In each case, the object is a finite sequence of terms over a particular set. Relevant properties, sometimes called "parameters", are the length of the sequence and, for integer compositions, the sum of the sequence.

There has been interest within enumerative combinatorics in counting words and compositions, especially restricted variations where the objects satisfy extra conditions. "Local" restrictions are related to contiguous subsequences, for example Smirnov words where adjacent letters must be different. For integer compositions or words over an ordered alphabet, a "subword pattern avoidance" restriction requires all contiguous subsequences of a fixed length to not satisfy a certain relative ordering. For example, we may count compositions not containing a strictly increasing contiguous subsequence of length three. "Global" restrictions, on the other hand, are related to arbitrary subsequences. A "subsequence pattern avoidance" restriction requires all subsequences of a fixed length to not satisfy a certain relative ordering.

Beyond sequences we may also consider objects with different structures, and interpret local and global restrictions appropriately. We say "cyclically restricted" finite sequences are those where the last and first terms are considered adjacent for the purposes of the restriction, i.e. the restriction wraps around from the end to the start. "Circular" objects are the orbits of finite sequences under circular shifts, so all circular shifts of a finite sequence are considered the same object.

We can generalize integer compositions by replacing the semigroup of positive integers with a different additive semigroup, giving the broader concept of a "composition over 
a semigroup", i.e. a finite sequence with a certain sum over the semigroup. Beyond the positive integers, we focus on semigroups which are finite groups - where such compositions are in fact also "words" in the group theory sense. Compositions over a finite group are relatively little-studied in combinatorics but turn out to be amenable to combinatorial analysis in analogy to both words and integer compositions.

In this document we achieve exact and asymptotic enumeration of words, compositions over a finite group, and/or integer compositions characterized by local restrictions and, separately, subsequence pattern avoidance. We also count cyclically restricted and circular objects. This either fills gaps in the current literature by e.g. considering particular new patterns, or involves general progress, notably with locally restricted compositions over a finite group. We associate these compositions to walks on a covering graph whose structure is exploited to simplify asymptotic expressions. Specifically, we show that under certain conditions the number of locally restricted compositions of a group element is asymptotically independent of the group element. For some problems our results extend to the case of a positive number of subword pattern occurrences (instead of zero for pattern avoidance) or convergence in distribution of the normalized number of occurrences. We typically apply the more general propositions to concrete examples such as the familiar Carlitz compositions or simple subword patterns. 


\section{Acknowledgements}

I thank Zhicheng Gao, not just for supervision on this thesis but as well for collaboration and opportunities over the years. I am also grateful for the suggestions and corrections of the thesis committee which includes Toufik Mansour, Mike Newman, Daniel Panario, and Michiel Smid. Professor Panario also played a big role in letting me get into combinatorics research.

This work was done in part on the premises of the Fields Institute for Research in Mathematical Sciences and the Massachussetts Institute of Technology. 


\section{Contents}

List of tables $\quad 1$

List of figures $\quad 2$

$\begin{array}{ll}\text { Notation } & 4\end{array}$

1 Introduction $\quad 6$

2 Locally restricted compositions $\quad 14$

2.1 Compositions over a finite group . . . . . . . . . . . . 15

2.2 Note on minimization of transfer matrices . . . . . . . . . . . 53

2.3 Note on weighted trees . . . . . . . . . . . . . . 56

3 Locally cyclically restricted compositions $\quad 60$

3.1 Compositions over a finite group . . . . . . . . . . . . . 60

3.2 Note on integer compositions . . . . . . . . . . . . . 66

4 Locally restricted compositions with symmetry $\quad 72$

4.1 Circular compositions . . . . . . . . . . . . . . . . . 72

4.2 Note on counting palindromic compositions . . . . . . . . . . . . 80

5 Subsequence pattern avoidance $\quad 82$

5.1 Words and integer compositions . . . . . . . . . . . . . 84

5.1.1 Pairs of generalized patterns of length $3 \ldots \ldots$. . . . . 85

5.1.2 Some partially ordered patterns with 2 letters . . . . . . . . 93

5.1.3 Note on counting with symmetries . . . . . . . . . . . 98

5.2 Note on compositions over $\mathbb{Z}_{k} \ldots \ldots \ldots$. . . . . . . . 101 
6 Conclusion

References 


\section{List of Tables}

1 Counts of Carlitz $m$-compositions of some $a$ over $S_{3}$. . . . . . . . 38

2 Counts of $m$-compositions of $a$ with no part followed by its inverse, over $Q_{8}\left(\right.$ written as a subgroup of $\left.S_{8}\right)$. . . . . . . . . . . . 43

3 Counts of $m$-compositions of some $a$ avoiding 132 over $\mathbb{Z}_{5}$. . . . . . 52

4 Counts of $m$-compositions of $a$ cyclically avoiding 132 over $\mathbb{Z}_{5} \ldots \ldots$

$5 \quad$ Counts of the $m$-compositions of $n$ avoiding $\{12-2,12-3\} \ldots$. . . . . 87

6 Counts of the $k$-ary $m$-words avoiding $\{21-2,2-12\} \ldots \ldots \ldots$

$7 \quad$ Counts of the $m$-compositions of $n$ avoiding $\{11-2,12-1\} \ldots \ldots 1$

8 Counts of $k$-ary $m$-words avoiding $\{12-3,3-21\} \ldots \ldots$

9 Counts of the $k$-ary words of length $m$ avoiding $2-1^{\prime}-1^{\prime \prime}-2 \ldots \ldots$. . . . 96

10 Counts of $m$-compositions of $a$ over $\mathbb{Z}_{4}$ avoiding $1^{\prime}-2-1^{\prime \prime} \ldots \ldots$. . . . 102 


\section{List of Figures}

1 Weighted digraphs $\Gamma$ (above) and $\Gamma^{\prime}$ (below). Integer vertex weights are shown below the corresponding vertices. . . . . . . . . . . . 8

2 Weighted digraphs $\Gamma$ (left) and $P$ (right). . . . . . . . . . . 9

3 A base digraph $D$ (left) and derived digraph $D_{\times}$(right) representing Carlitz compositions over $\mathbb{Z}_{3}$. Here all vertices of $D$ are allowed start and finish vertices. Vertices in $D_{\times}$that are allowed start vertices are shown with a double circle. . . . . . . . . . . . . . 18

4 Uniform-randomly generated Carlitz 100-compositions of 0 (above) and 1 (below) over $\mathbb{Z}_{3}$. (The vertical axis represents the value of a part, i.e. the below composition starts $(1,2,0,2, \ldots)$. $\quad$. . . . . 20

$5 \quad$ A digraph $D$ with vertices in $\mathbb{Z}_{4}^{2} \ldots \ldots . \ldots . . \ldots 20$

6 An aperiodic strongly connected digraph $D$ (above) with vertices in $\mathbb{Z}_{4}^{2}$ such that $D_{\times}$(one component shown below) has period 2. Examples with connected $D_{\times}$exist as well, such as the above $D$ over $\mathbb{Z}_{8}$ with 7

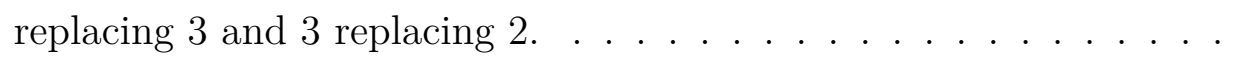

7 A base digraph $D$ (above) and derived digraph $D_{\times}$with 2 strong components (below). The vertices of $D$ are 4 -tuples over $\mathbb{Z}_{2}$. . . . . . . . 32

8 Strong components in the proof of Lemma 2.4. . . . . . . . . . . . 34

9 Uniform-randomly generated 2-Carlitz 100-compositions of 0 (above) and 1 (below) over $\mathbb{Z}_{5}$. . . . . . . . . . . . . . . . 38

10 Uniform-randomly generated 100-compositions of 0 (above) and 1 (below) over $\mathbb{Z}_{5}$ with $x(i) \neq-x(i+1)$. . . . . . . . . . 41

11 Uniform-randomly generated 100-compositions of 0 (above) and 1 (below) over $\mathbb{Z}_{5}$ which avoid $132 . \quad \ldots \ldots \ldots \ldots$ 
12 Uniform-randomly generated 100-compositions of 0 (above) and 1 (below) over $\mathbb{Z}_{5}$ which avoid $121 . \ldots \ldots \ldots . \ldots . \ldots . . \ldots 53$

13 A DFA that accepts 3-ary Carlitz words over $\{a, b, c\} . \ldots 54$

14 A DFA weakly equivalent to one that accepts 3-ary Carlitz words. . 54

15 A tree weighted by $\mathbb{Z}_{4}$ shown in two plotting styles. . . . . . . . . . 58

16 Uniform-randomly generated 100-compositions of 0 (above) and 1 (below) over $\mathbb{Z}_{5}$ which cyclically avoid 132 . . . . . . . . . . 65

17 Integer composition of 300 avoiding the subword pattern 123 generated by 10,000,000 iterations of an MCMC method. . . . . . . . . . . . . 71

18 Uniform-randomly generated compositions of 150 avoiding

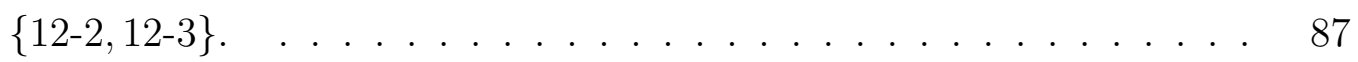

19 Uniform-randomly generated 10-ary (above) and 40-ary (below) 100words avoiding $\{21-2,2-12\} \ldots \ldots \ldots$. . . . . . . . . . 90

20 Uniform-randomly generated compositions of 50 avoiding $\{11-2,12-1\} . \quad 91$

21 Uniform-randomly generated $k$-ary 100 -words where $k=3,4,5,20$

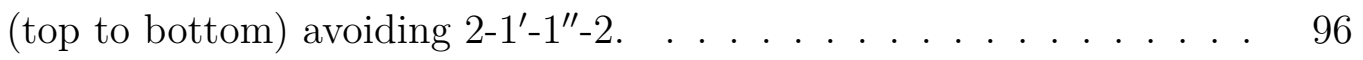

22 Uniform-randomly generated compositions of 150 avoiding $1^{\prime}-1^{\prime \prime}-2 . \quad$. 98 


\section{Notation}

All $n$-tuples over set $\Xi\left[\right.$ FS09]: $\operatorname{SEQ}_{n}(\Xi)$

All finite sequences over set $\Xi: \operatorname{SEQ}(\Xi)=\cup_{n} \operatorname{SEQ}_{n}(\Xi)$

Asymptotic equivalence; $f(n)$ asymptotic to $g(n): \lim _{n \rightarrow \infty} f(n) / g(n)=1 \Longleftrightarrow$ $f(n) \sim g(n)$

Big-Oh; there is $c>0$ such that for all sufficiently large $n,|f(n)| \leq c g(n): f(n)=$ $O(g(n))$

Big-Theta; there are $c, d>0$ such that for all sufficiently large $n,|f(n)| \leq c g(n)$ and $|f(n)| \geq d g(n): f(n)=\Theta(g(n))$

Cardinality: $|\Xi|$

Closed neighborhood: $N[v]$, open neighborhood (excludes $v$ unless there is a loop) $[\mathrm{BM} 76]: N(v)$

Concatenation of finite sequences: $(a, b) \frown(c, d)=(a, b, c, d)$

Convergence in distribution, weak convergence: $\Rightarrow$

Disjoint union: $\dot{U}$

Falling factorial: $n^{\underline{k}}=n(n-1) \cdots(n-k+1)$

Finite cyclic groups: $\mathbb{Z}_{k}=\{0, \ldots, k-1\}$

Finite sequence short form: $1^{4} 23^{2}=(1,1,1,1,2,3,3)$ 
First $k$ positive integers: $[k]=\{n: 1 \leq n \leq k, n \in \mathbb{Z}\}$

In-neighborhoods (open and closed) [BM76]: $\quad N^{-}(v), N^{-}[v]$, out-neighborhoods: $N^{+}(v), N^{+}[v]$

Iverson bracket; 1 if the statement $\phi$ is true and 0 otherwise [Knu92]: $[\phi]$

Matrix entry: $[M]_{i, j}$

Matrix/vector literal: $\left[\begin{array}{l}1 \\ 2\end{array}\right]=\left[\begin{array}{ll}1 & 2\end{array}\right]^{\top}$

Non-negative integers: $\mathbb{Z}_{\geq 0}=\{0,1,2, \ldots\}$

Normal distribution function with mean $\mu$, variance $\sigma^{2}: N\left(\mu, \sigma^{2}\right)$

Partial derivative of power series with respect to indeterminate $u$ : $D_{u} f$

Positive integers: $\mathbb{Z}_{>0}=\{1,2,3, \ldots\}$

Reversal of finite sequence: if $a=(a(1), \ldots, a(m))$, then $\stackrel{\leftarrow}{a}=(a(m), \ldots, a(1))$

Stirling subset numbers (Stirling numbers of the second kind) [GKP94, p. 258]:

$\left\{\begin{array}{c}m \\ k\end{array}\right\}$

Subset: $\subseteq$, strict subset: $\subset$

Sum of finite sequence $a$ : $\Sigma a$ (capital sigma)

Transitive closure of arc relation: $\longrightarrow$ (long arrow) 


\section{Introduction}

If $\Xi$ is a finite set (sometimes called an alphabet), a word $w$ over $\Xi$ is a sequence $w=(w(1), \ldots, w(m))$ where $w(i) \in \Xi$ for each $i$. In particular, if $|\Xi|=k$ we call $w$ a $k$-ary $m$-word. Without loss of generality, if $|\Xi|=k$ we assume $\Xi=[k]=\{1, \ldots, k\}$, which is an alphabet with a total order. The terms that make up a word are called letters. Of course the number of all $k$-ary $m$-words is $k^{m}$.

If $(S,+)$ is a semigroup, an $m$-composition of $s \in S$ over $S$ is a sequence $x=$ $(x(1), \ldots, x(m))$ where $x(i) \in S$ for each $i$, and $\Sigma x=x(1)+\cdots+x(m)=s$. If $S$ is finite, a composition over $S$ and a word over $S$ mean the same thing; the difference is that we use the word composition in the context where we pay attention to the sum of the sequence. The prototypical compositions are integer compositions, where $(S,+)=\left(\mathbb{Z}_{>0},+\right)$. The terms that make up a composition are called parts. A simple exercise gives that the number of $m$-compositions of $n$ over the positive integers is $\left(\begin{array}{l}n-1 \\ m-1\end{array}\right)$.

A subword of a finite sequence is a contiguous subsequence, so $(a, a, c, b)$ is a subword of $(a, a, a, a, c, b, b)$. For any kind of finite sequences we may sometimes use the shorthand word notation $(a, a, a, a, c, b, b)=a^{4} c b^{2}=a a a a c b b$.

A directed graph (digraph) is a pair $(V, E)$, where $V$ is a finite set (the "vertices"), and $E \subseteq V \times V$ is a binary relation (the "directed edges" or "arcs"). If a digraph is specified only by its arcs, the vertices are taken to be all those which appear in an

arc. A weighted digraph is a digraph $(V, E)$ together with a vertex weight function $W: V \rightarrow S$, where $S$ is a fixed semigroup. Words and compositions are both finite sequences over a set. Equivalently, we may regard them as directed paths (digraphs 
with an ordered set of vertices and arcs from predecessors to successors) where vertices take weights from the set (which is taken without loss of generality to be a semigroup). The benefit of this view comes when generalizing beyond directed paths to different types of weighted digraphs.

Our goal, ultimately, is to count weighted digraphs. Specifically, we are interested in counting how many of these objects satisfy a certain restriction. Below, we describe a general concept of pattern occurrence and avoidance in weighted digraphs which we can use to express restricted weighted digraph families. The familiar definitions of patterns in words and compositions (e.g. [HM10]) are available as special cases.

If $\Gamma$ is a digraph, we write $V(\Gamma)$ and $E(\Gamma)$ for the sets of vertices and arcs of $\Gamma$. Given digraphs $\Gamma_{1}, \Gamma_{2}$, a digraph homomorphism is a function $h: V\left(\Gamma_{1}\right) \rightarrow V\left(\Gamma_{2}\right)$ such that for any two vertices $u, v \in V\left(\Gamma_{1}\right)$, we have

$$
(u, v) \in E\left(\Gamma_{1}\right) \Longrightarrow(h(u), h(v)) \in E\left(\Gamma_{2}\right) .
$$

If $\Gamma_{1}, \Gamma_{2}$ have weight functions $W_{1}, W_{2}$, a weighted homomorphism is a homomorphism $h$ such that $W_{1}(v)=W_{2}(h(v))$ for all $v \in V\left(\Gamma_{1}\right)$. A (weighted) isomorphism is a bijective (weighted) homomorphism.

Notation 1.1. We use the notation $A \cup \dot{\cup}$ to denote the union of the disjoint sets $A$ and $B$.

A one-vertex subdivision of a digraph $(V, E)$ is a new digraph $\left(V^{\prime}, E^{\prime}\right)$, where $V^{\prime}=$ $V \dot{\cup}\{v\}$ and for some $\left(v_{1}, v_{2}\right) \in E$, we have

$$
E^{\prime}=\left\{\left(v_{1}, v\right),\left(v, v_{2}\right)\right\} \cup E \backslash\left\{\left(v_{1}, v_{2}\right)\right\}
$$

A weighted one-vertex subdivision is one where the weight function is not modified 


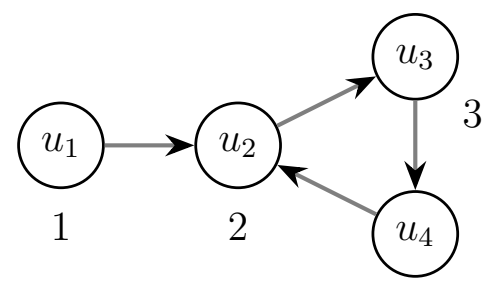

1

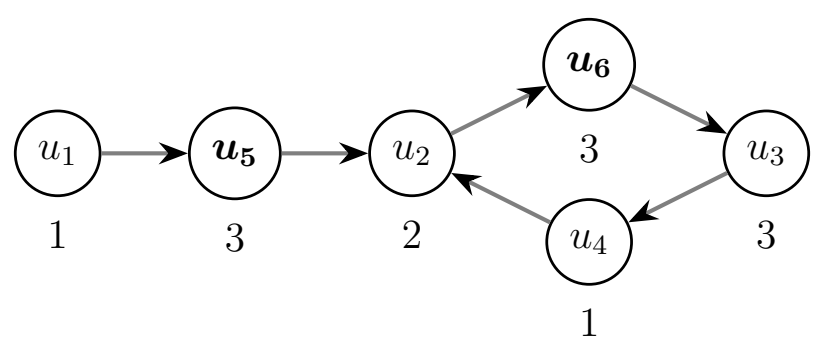

Figure 1: Weighted digraphs $\Gamma$ (above) and $\Gamma^{\prime}$ (below). Integer vertex weights are shown below the corresponding vertices.

except for the new vertex $v$, which may take any weight. In general, a subdivision of a digraph is a digraph obtained by 0 or more one-vertex subdivisions. In the context of weighted graphs, subdivisions are always weighted.

Example 1.1. Figure 1 shows digraphs $\Gamma, \Gamma^{\prime}$. The digraph $\Gamma^{\prime}$ is a subdivision of $\Gamma$ obtained by adding the vertices $u_{5}, u_{6}$ which are shown in bold.

Given weighted digraphs $\Gamma, P$, a subdivision $P^{\prime}$ of $P$, and a subgraph $\Gamma_{1}$ of $\Gamma$, if we have a weighted isomorphism $f: V\left(P^{\prime}\right) \rightarrow V\left(\Gamma_{1}\right)$, we say that $f_{\mid V(P)}$ is the match of $f$ with respect to $P$. A local occurrence of $P$ in $\Gamma$ is the match with respect to $P$ of some weighted $f$ from $P$ to a subgraph of $\Gamma$. A global occurrence of $P$ is the match with respect to $P$ of some weighted $f$ from any subdivision $P^{\prime}$ of $P$ to a subgraph of $\Gamma$.

That is, global occurrences may map adjacent vertices in $P$ to non-adjacent vertices in $\Gamma$ while local occurrences cannot. The semigroup $S$ of weights is always the same 


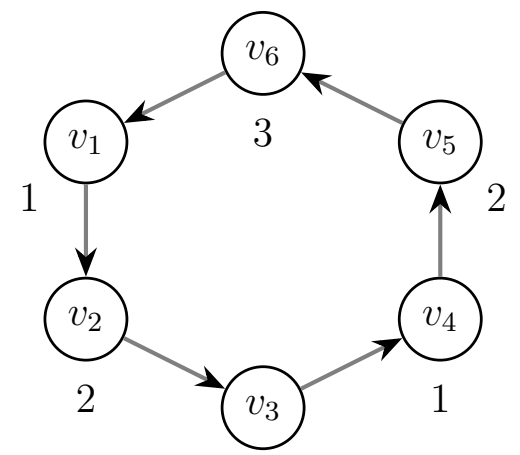

3

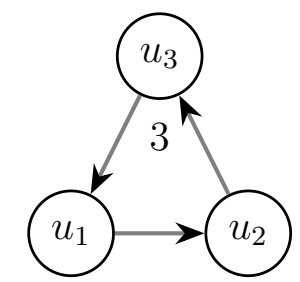

1

2

Figure 2: Weighted digraphs $\Gamma$ (left) and $P$ (right).

for $\Gamma$ and $P$.

Example 1.2. Figure 2 shows weighted digraphs $\Gamma$ and $P$. There exist no local occurrences of $P$ in $\Gamma$ but many global occurrences. One global occurrence is given by matching the vertices

$$
u_{1} \mapsto v_{1}, u_{2} \mapsto v_{2}, u_{3} \mapsto v_{3}
$$

Another is given by

$$
u_{1} \mapsto v_{1}, u_{2} \mapsto v_{5}, u_{3} \mapsto v_{6}
$$

The size of a digraph is the number of arcs it contains. A digraph pattern is a set $\mathcal{P}$ of weighted digraphs such that the sizes of digraphs in $\mathcal{P}$ form a bounded set. The elements $P \in \mathcal{P}$ are pattern instances and for our purposes, the instances of $\mathcal{P}$ are always one or more different weightings of a single digraph. A digraph $\Gamma$ has $r$ occurrences of $\mathcal{P}$ if the total, over all instances $P \in \mathcal{P}$, of the number of occurrences of $P$ in $\Gamma$ is $r$. Avoiding a digraph pattern means having 0 occurrences, and avoidance of a set of patterns means avoiding each of the patterns.

Given an arbitrary finite vertex set, say $V=[n]$, an unlabeled weighted digraph $\tilde{\Gamma}$ on 
$V$ is an equivalence class of weighted digraphs on $V$ where $\Gamma_{1}$ and $\Gamma_{2}$ are equivalent if there is a weighted isomorphism from $\Gamma_{1}$ to $\Gamma_{2}$. Relabeling vertices has no effect on digraph pattern matching because it does not affect the structure of the digraph or its weights, so we may speak of the number of occurrences of a pattern in an unlabeled weighted digraph.

The sum, a.k.a. total, of a weighted digraph $\Gamma$ is $\sum_{v \in V(\Gamma)} W(v)$. This expression is always well defined for abelian semigroups $S$. For non-abelian $S$ we must have labeled digraphs, and the vertices must have a fixed total order which determines the order of summation.

Example 1.3. Define the set of directed paths

$$
\{\{(j, j+1): 1 \leq j<n\}: n \geq 1\}
$$

and define the set of directed cycles

$$
\{\{(j, j+1): 1 \leq j<n\} \cup\{(n, 1)\}: n \geq 1\} .
$$

With the terminology of [HM10] we may make the following identifications. Weighted paths are words or compositions, and weighted cycles are cyclic words or compositions (where the last term is considered to precede the first for pattern occurrence purposes). Unlabeled weighted cycles over the vertex sets $[n]$ correspond to circular words or compositions.

In the remainder, "path" means directed path and "cycle" means directed cycle.

With the above concepts laid down we are able to describe a wide taxonomy of counting problems which all ask, how many weighted digraphs are there with $r$ occurrences of a digraph pattern $\mathcal{P}$ ? The primary dimensions of this taxonomy follow. 
- Class of digraphs. There are many options for the kind of digraph that we are counting. Paths and cycles are the most basic, but others could be used: regular, planar, bipartite, et cetera. The elements of $\mathcal{P}$ are also digraphs and can come in any form. We largely focus on digraph patterns $\mathcal{P}$ that consist of various weightings of a path.

- Labeled vs. unlabeled. The digraphs we count may be either labeled or unlabeled. Usually counting the labeled case is a prerequisite for the unlabeled case.

- Local vs. global occurrences. If we ask for $r$ occurrences, we are either talking about local or global occurrences.

- Track size, total, or both. When counting words, any algebraic structure of the alphabet is ignored, unlike with compositions. Similarly for digraphs, we may or may not keep track of the total.

- Choice of semigroup. Any semigroup could be used as long as it gives finite counts, e.g. the number of 6-compositions of 17 over $\mathbb{Z}$ is infinite.

The remainder of this document contains solutions to a selection of problems from the taxonomy just described. We largely defer discussions of the relevant prior literature to the sections that follow due to their heterogeneity. However, we mention here the 2010 book [HM10] by Heubach and Mansour which is a useful reference for many of the topics of this document, especially for exact (as opposed to asymptotic) counting. The remaining sections of this document are organized as follows.

We begin in $\S 2.1$ with local occurrences in weighted paths, where the semigroup $S$ is a finite group. A weighted path with no local occurrences of some pattern is known as a locally restricted composition, assuming we track the total. (In this and 
subsequent sections we generally use the familiar concepts, such as "compositions", although we refer to weighted digraphs when useful.) We find, under conditions, that the number of locally restricted compositions of a group element is asymptotically independent of the group element. We reach the same conclusion for compositions containing $r>0$ local occurrences of a pattern. After verifying these conditions for a variety of examples, we show that under similar conditions the number of local occurrences in a random composition is asymptotically normal. In $\S 2.2$ we make a note on when the matrices used in the transfer matrix method can be reduced in size for computational and practical benefits. This section and others with heading "Note on..." are extended remarks which briefly introduce relevant lines of research. The problem of counting directed rooted trees is noted in $\S 2.3$, also in the context of local pattern occurrence.

Next, $§ 3.1$ is similar to $\S 2.1$ but counts digraphs which are cycles rather than paths, which correspond to objects known as locally cyclically restricted compositions. Again we find that under conditions the asymptotic number of such compositions of a finite group element does not depend on the group element, and we show asymptotic normality of the number of local pattern occurrences. In $\S 3.2$ we note how to count locally cyclically restricted integer compositions, i.e. cycles weighted by $\mathbb{Z}_{>0}$, in the framework of locally restricted integer compositions of [BC09].

The results of $\S 4.1$ together with Moebius inversion allow us to count circular locally restricted compositions over a finite group which is done in §4.1. As in Example 1.3, circular objects correspond to unlabeled weighted cycles. In $\S 4.2$ we note how to count "undirected" locally restricted compositions, i.e. unlabeled weighted undirected paths. 
"Subsequence patterns" and "generalized patterns" are types of patterns that are used in the context of ordered semigroups $S$. In the language of this section they are digraph patterns made up of all paths that have a certain size and a certain relative ordering among the vertex weights. "Partially ordered patterns" can be used to represent a set of subsequence patterns. Subsequence and partially ordered patterns are used in the context of global occurrences, while generalized patterns actually specify which arcs may be subdivided and which may not. In $\$ 5.1 .1$ we count weighted paths, specifically words or integer compositions, that avoid different pairs of generalized patterns. The counting results in $\$ 5.1 .2$ are concerned with words or integer compositions that avoid a family of partially ordered patterns (roughly, patterns where the maximal weights must be at the beginning or end). In $\$ 5.1 .3$ we note how to adapt results for subsequence pattern avoidance in words to circular words (unlabeled weighted cycles) or "undirected" words (unlabeled weighted undirected paths). We make a note on subsequence pattern avoidance in objects other than words and integer compositions in $\$ 5.2$, namely in compositions over $\mathbb{Z}_{k}$. Our technique involves using the multisection formula together with results for integer compositions, and we apply it to an example partially ordered pattern.

Finally, we list open problems in $\S 6$. 


\section{Locally restricted compositions}

A locally restricted composition is one that avoids a certain set of length- $l$ sequences as subwords. Over the integers, these objects have been studied successfully in a number of papers by Bender et al. [BC09; BCG12; BG14]. In fact, those works include somewhat more general restrictions, where a subword may or may not be allowed based on the residue of its position in a composition, and special rules can apply to parts near the beginning or end. Under some conditions, asymptotics for the number of locally restricted integer compositions were given in [BC09]. That paper also established a normal limiting distribution for the number of occurrences of a subword in a uniform random integer composition. The later papers [BCG12; BG14] focused on the probability distributions of part sizes and other parameters.

Given two sequences $x, y$ of the same length over ordered sets, we say $x$ and $y$ are order isomorphic if $x(i)<x(j) \Longleftrightarrow y(i)<y(j)$ for all $i, j$. A subword pattern $\tau$ is a word over $[k]$. Assume the length of $\tau$ is $l$. An occurrence of $\tau$, as a subword pattern, in $x$ is a sequence of indices $i, \ldots, i+l-1$ such that $(x(i), \ldots, x(i+l-1))$ is order isomorphic to $\tau$.

Mansour and others in [BM03; MS06; HM10] count integer compositions by number of occurrences of specific subword patterns such as 123 and 112. These results are less general than those obtained by Bender and collaborators in the case of avoidance but give simpler expressions. The umbral technique in [Zei00] is also used to explicitly count locally restricted objects.

Remark 2.1. In the language of $\S 1$, compositions are weighted directed paths where we keep track of the total weight. Weighted undirected paths may be counted in a 
similar manner.

\subsection{Compositions over a finite group}

Locally restricted compositions over finite fields and even finite abelian groups were counted in [GMW18] under some conditions, and in less generality in the preceding papers mentioned therein. Over $\mathbb{Z}_{k}$, the method used in that paper involves obtaining the relevant generating function $F(z)$ for integer compositions over $[k]$, and working with $\sum_{j \equiv s(\bmod k)}\left[z^{j}\right] F(z)$. For other finite abelian groups, the method is extended to multivariate generating functions. Below we give an alternative counting method that expands the range of applicability beyond abelian groups, addressing a problem posed in [GMW18]. We begin this section considering compositions over a finite semigroup $(S,+)$ and eventually specialize to finite groups.

Notation 2.1. For a finite set $\Xi$, we denote all n-tuples over $\Xi$ by $\operatorname{SEQ}_{n}(\Xi)$. We define $\operatorname{SEQ}(\Xi)=\cup_{n \geq 0} \operatorname{SEQ}_{n}(\Xi)$.

Definition 2.1. Let $\Xi$ be a finite set, and let $n$ be a positive integer. The $n$ dimensional de Bruijn graph (actually a digraph) on $\Xi$ has vertex set $V=\operatorname{SEQ}_{n}(\Xi)$ and includes the arc from $\left(u_{1}, \ldots, u_{n}\right)$ to $\left(v_{1}, \ldots, v_{n}\right)$ if and only if

$$
\left(u_{2}, \ldots, u_{n}\right)=\left(v_{1}, \ldots, v_{n-1}\right)
$$

Notation 2.2. We use the symbol $\frown$ to denote concatenation of finite sequences, e.g. $(a, b) \frown(c, d)=(a, b, c, d)$

Let $\sigma$ be a positive integer which we call the span, and let $D$ be a subgraph of the $\sigma$-dimensional de Bruijn graph on $S$. Then $D$ is a de Bruijn subgraph. This digraph 
$D$ is associated with a set of locally restricted compositions as follows. A walk in a digraph is a sequence of vertices, not necessarily distinct, where for any subword $(u, v)$ there is an arc from $u$ to $v$. An $m$-composition over $S$ is legal according to $D$ if it takes the form

$$
w_{1} \frown\left(w_{2}(\sigma), \ldots, w_{m-\sigma+1}(\sigma)\right)=\left(w_{1}(1), \ldots, w_{1}(\sigma), w_{2}(\sigma), \ldots, w_{m-\sigma+1}(\sigma)\right)
$$

where $w_{1}, \ldots, w_{m-\sigma+1}$ is a walk in $D$. In other words, we build compositions from $D$ by starting at any vertex, and taking a walk in which we append the last element of each vertex we visit after the first vertex. Additionally, we may designate sets of start and end vertices which are the allowed vertices for walks to start and end at.

We write the set of all $m$-compositions of $s$ that are legal according to $D$ with start set $\Psi$ and finish set $\Phi$ as $\mathcal{P}_{s}(m ; D, \Psi, \Phi)$. We may also write this with $s, m, \Psi$, or $\Phi$ omitted to remove those conditions, e.g. $\mathcal{P}_{s}(D, \Psi, \Phi)=\cup_{m} \mathcal{P}_{s}(m ; D, \Psi, \Phi)$. Also, $p_{s}(m ; D, \Psi, \Phi)=\left|\mathcal{P}_{s}(m ; D, \Psi, \Phi)\right|, P_{s}(z ; D, \Psi, \Phi)=\sum_{m \geq 0} p_{s}(m ; D, \Psi, \Phi) z^{m}$.

Define a new digraph $D_{\times}$with vertex set $V(D) \times S$ such that $((u, s),(v, t)) \in E\left(D_{\times}\right)$ if and only if $(u, v) \in E(D)$ and $s+v(\sigma)=t$. We call $D_{\times}$the derived digraph (of the base digraph $D)$. We define the start set $\Psi_{\times} \subseteq V\left(D_{\times}\right)$to contain all $(v, s)$ such that $\sum v=s$ and $v \in \Psi$. For each $s \in S$ the finish set $\Phi_{s} \subseteq V(D)$ for $s$ contains all vertices $(v, s)$ where $v \in \Phi$.

Fix an ordering on $V\left(D_{\times}\right)$so we can define an adjacency matrix $M_{\times}$of $D_{\times}$. Let $\psi_{\times} \in \mathbb{R}^{\left|V\left(D_{\times}\right)\right|}$be the indicator vector for $\Psi_{\times}$, and let $\phi_{s} \in \mathbb{R}^{\left|V\left(D_{\times}\right)\right|}$be the indicator vector for $\Phi_{s}$. 
Proposition 2.1. For $m \geq \sigma$, we have

$$
p_{s}(m ; D, \Psi, \Phi)=\psi_{\times}^{\top} M_{\times}^{m-\sigma} \phi_{s} .
$$

The generating function $P_{s}(z ; D, \Psi, \Phi)$ is rational.

Proof. Let $W_{s}$ be a walk in $D_{\times}$starting in $\Psi_{\times}$and ending in $\Phi_{s}$ in the form

$$
W_{s}=\left(\left(w_{1}, t\right), \ldots,\left(w_{m-\sigma+1}, s\right)\right),
$$

so the $D$-vertices corresponding to $W_{s}$ are $w_{1}, \ldots, w_{m-\sigma+1}$. We say the $m$-composition of $s$ defined by $W_{s}$ is

$$
w_{1} \frown\left(w_{2}(\sigma), \ldots, w_{m-\sigma+1}(\sigma)\right) .
$$

By the definition of $D_{\times}$, the $m$-compositions corresponding to a walk $W_{s}$ in $D_{\times}$are exactly those $m$-compositions allowed by $D$ with total $s$. That is, the compositions defined by $D_{\times}$and $D$ are the same, but $D_{\times}$also directly keeps track of the total.

Counting walks in a digraph via the adjacency matrix is a well-known procedure. The result follows from the relation

$$
\left[M_{\times}^{q}\right]_{i, j}=\sum_{k=1}^{\left|V\left(D_{\times}\right)\right|}\left[M_{\times}\right]_{i, k}\left[M_{\times}^{q-1}\right]_{k, j}
$$

which means walks of length $q+1$ from vertex $i$ to $j$ are walks of length 2 from $i$ to $k$, merged with a walk of length $q$ from $k$ to $j$. This is known as the transfer matrix method; background may be found in [Sta12]. 


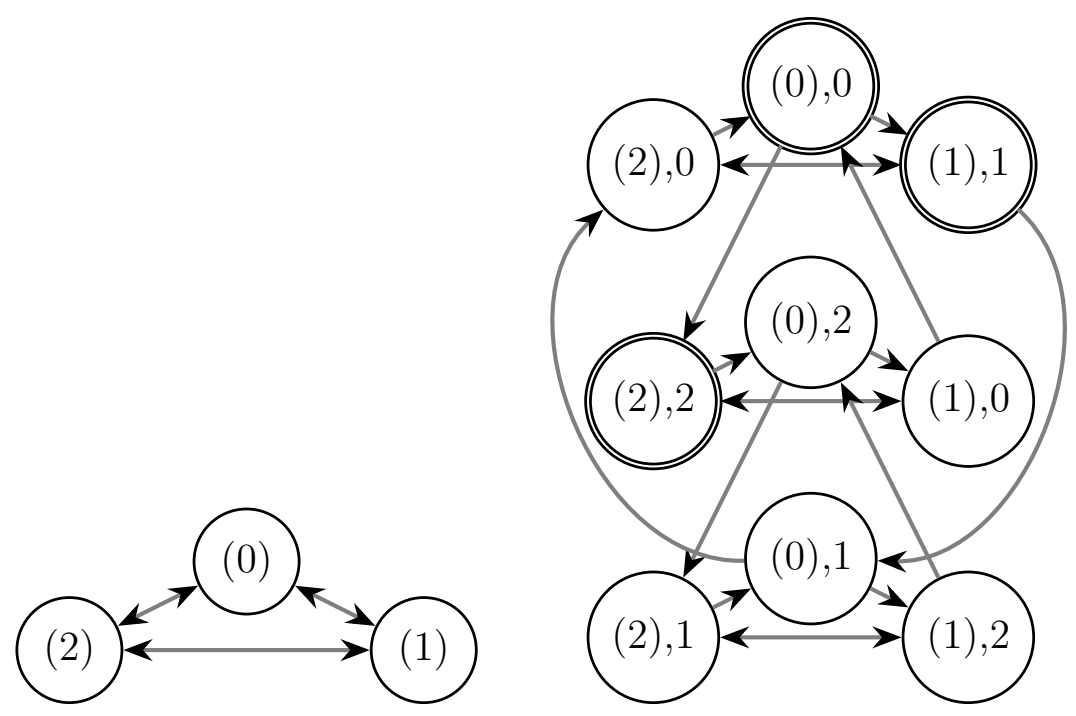

Figure 3: A base digraph $D$ (left) and derived digraph $D_{\times}$(right) representing Carlitz compositions over $\mathbb{Z}_{3}$. Here all vertices of $D$ are allowed start and finish vertices. Vertices in $D_{\times}$that are allowed start vertices are shown with a double circle.

We have

$$
\begin{aligned}
P_{s}(z ; D, \Psi, \Phi) & =\sum_{m \geq \sigma} \psi_{\times}^{\top} M_{\times}^{m-\sigma} \phi_{s} z^{m}+P(z) \\
& =z^{\sigma} \psi_{\times}^{\top}\left(\sum_{m \geq 0} M_{\times}^{m} z^{m}\right) \phi_{s}+P(z) \\
& =z^{\sigma} \psi_{\times}^{\top}\left(I-z M_{\times}\right)^{-1} \phi_{s}+P(z),
\end{aligned}
$$

where $P(z)$ is a polynomial which counts the appropriate $m$-compositions with $m<\sigma$. The entries of $\left(I-z M_{\times}\right)^{-1}$ lie in the field of fractions of $\mathbb{Q}[z]$, i.e. the rational functions $\mathbb{Q}(z)$.

Example 2.1. Carlitz compositions are those where adjacent parts must be different. Figure 3 shows an example of $D_{\times}$for Carlitz compositions over $\mathbb{Z}_{3}$.

Let us order the vertices of $D_{\times}$as

$$
((0), 0),((1), 1),((2), 0),((0), 2),((1), 0),((2), 2),((0), 1),((1), 2),((2), 1) \text {. }
$$


Then we get

$$
M_{\times}=\left[\begin{array}{ccccccccc}
0 & 1 & 0 & 0 & 0 & 1 & 0 & 0 & 0 \\
0 & 0 & 1 & 0 & 0 & 0 & 1 & 0 & 0 \\
1 & 1 & 0 & 0 & 0 & 0 & 0 & 0 & 0 \\
0 & 0 & 0 & 0 & 1 & 0 & 0 & 0 & 1 \\
1 & 0 & 0 & 0 & 0 & 1 & 0 & 0 & 0 \\
0 & 0 & 0 & 1 & 1 & 0 & 0 & 0 & 0 \\
0 & 0 & 1 & 0 & 0 & 0 & 0 & 1 & 0 \\
0 & 0 & 0 & 1 & 0 & 0 & 0 & 0 & 1 \\
0 & 0 & 0 & 0 & 0 & 0 & 1 & 1 & 0
\end{array}\right], \psi_{\times}=\left[\begin{array}{c}
1 \\
1 \\
0 \\
0 \\
0 \\
1 \\
0 \\
0 \\
0
\end{array}\right], \phi_{0}=\left[\begin{array}{c}
1 \\
0 \\
1 \\
0 \\
1 \\
0 \\
0 \\
0 \\
0
\end{array}\right],
$$

So the number of Carlitz 3-compositions of 0 in $\mathbb{Z}_{3}$ is 6 .

Remark 2.2. The following procedure generates a walk in $D_{\times}$of length $m-\sigma+1$, where all such walks are equally probable:

1. Pick a start vertex $v_{1}$ weighted by the number of $(m-\sigma+1)$-walks from that vertex to a finish vertex.

2. Given the current vertex $v_{i}$, select an out-neighbor where such neighbors are weighted by the number of $(m-\sigma+1-i)$-walks from the neighbor vertex to a finish vertex.

Naturally, using the correspondence between walks and compositions, this gives a method of random generation for locally restricted compositions. Figure 4 shows an example with Carlitz compositions. Other examples of the method are found throughout this section.

If $D_{\times}$is strongly connected and aperiodic, then we can obtain a highly-precise asymptotic expression for $p_{s}(m ; D, \Psi, \Phi), m \rightarrow \infty$, via Proposition 2.1 and the Perron- 


\section{".

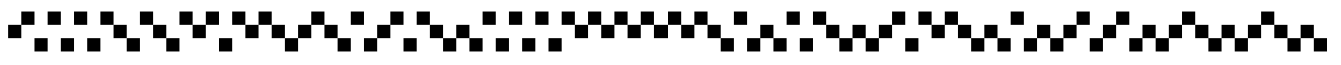

Figure 4: Uniform-randomly generated Carlitz 100-compositions of 0 (above) and 1 (below) over $\mathbb{Z}_{3}$. (The vertical axis represents the value of a part, i.e. the below composition starts $(1,2,0,2, \ldots)$.)

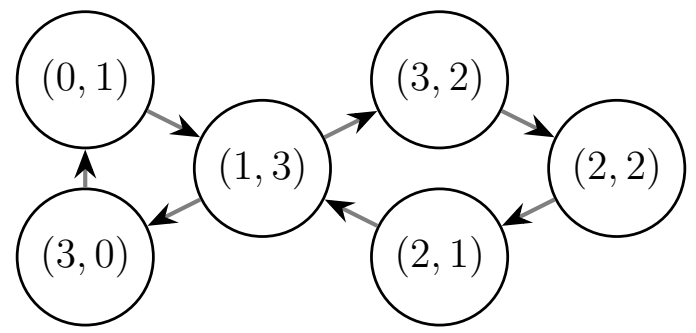

Figure 5: A digraph $D$ with vertices in $\mathbb{Z}_{4}^{2}$.

Frobenius theorem. (A digraph is aperiodic if the set of all cycle lengths has no common divisor besides 1.) We now give some general facts about the strong connectedness of $D_{\times}$.

If $D$ is not strongly connected then certainly $D_{\times}$is not strongly connected either. However, if $D$ decomposes into disconnected strong components, then naturally we are able to simply count with each component separately and add. In the following, we assume $D$ is strongly connected (and nonempty).

Unfortunately, if $D$ is strongly connected, $D_{\times}$is not necessarily strongly connected. Say $D$ is the digraph given in Figure 5 , over $\mathbb{Z}_{4}$ with $\operatorname{span} \sigma=2$. In $D_{\times}$, there is a path from $((3,0), 3)$ to $((1,3), 3)$, but there is no path from $((1,3), 0)$ to $((1,3), 3)$.

If the entirety of $D_{\times}$is not strongly connected then we would hope it is simply a disjoint union of strong components. This is not true for general finite semigroups $S$. 
For example, if there is $s^{*} \in S$ satisfying

$$
\forall s \in S: s^{*}+s=s+s^{*}=s^{*},
$$

then walks in the digraph $D_{\times}$will get "stuck" at $s^{*}$ and some weakly connected vertices will not be strongly connected. We do obtain this desideratum, however, if $S$ is a group, as we show eventually below. In the following we assume that $S=G$ is a group.

Definition 2.2. Let $D_{B}$ be an arbitrary digraph, referred to as the base digraph. Let $G$ be a finite group, and let $\alpha: E\left(D_{B}\right) \rightarrow G$ map arcs of $D_{B}$ to group elements. Together, $D_{B}$ and $\alpha$ are known as a voltage graph. We define the derived digraph $D_{\alpha}$ such that $V\left(D_{\alpha}\right)=V\left(D_{B}\right) \times G$ and $((u, a),(v, b))$ is an arc if and only if $(u, v) \in E\left(D_{B}\right)$ and $a+\alpha(u, v)=b$.

The digraphs $D_{\times}$directly give derived digraphs in the sense of voltage graphs, specifically "right derived ordinary voltage graphs", if we associate the group element $u(\sigma)$ to all incoming arcs to $u$ in the base digraph $D$.

Notation 2.3. If $v$ is a vertex in a digraph with arc relation $E$, we use the following notations:

$$
\begin{aligned}
N^{-}(v) & =\{u:(u, v) \in E\} \\
N^{-}[v] & =N^{-}(v) \cup\{v\} \\
N^{+}(v) & =\{u:(v, u) \in E\} \\
N^{+}[v] & =N^{+}(v) \cup\{v\} \\
N(v) & =N^{-}(v) \cup N^{+}(v) \\
N[v] & =N^{-}[v] \cup N^{+}[v] .
\end{aligned}
$$


Remark 2.3. Let $\left(V_{1}, E_{1}\right)$ and $\left(V_{2}, E_{2}\right)$ be graphs. Then $\left(V_{2}, E_{2}\right)$ is a covering graph of $\left(V_{1}, E_{1}\right)$ if there is a surjection $f: V_{2} \rightarrow V_{1}$ such that for each $v \in V_{2}$, the restriction $f_{\mid N[v]}$ is a bijection. In that case, $f$ is called a covering map. We note that derived graphs can be seen as a covering graphs of the base graph, but directed. The book [GT87] provides a basic introduction to covering graphs in Chapter 2. Covering graphs are more generally known as covering spaces in topology.

Notation 2.4. For vertices $u$ and $v$, we use the notation $u \longrightarrow v$ to denote that there exists a directed walk from $u$ to $v$.

Lemma 2.1. The derived digraph $D_{\times}$is a disjoint union of strong components.

Proof. Select a vertex $(u, a)$, and take another vertex $(v, b)$ such that there is a path $(u, a) \longrightarrow(v, b)$ in $D_{\times}$. Since $D$ is strongly connected, there is a path $(v, b) \longrightarrow(u, c)$ in $D_{\times}$for some $c \in G$. This implies that $(u, a) \longrightarrow(u, c)$. We are done if we can show that $(u, c) \longrightarrow(u, a)$.

Since there is a path $(u, a) \longrightarrow(u, c)$, we know that for any positive integer $j$, there is a path $(u, a) \longrightarrow(u, a+j(-a+c))$, which is found by repeating the path in $D$. In a finite digraph we will eventually get $g>j>0$ with $a+j(-a+c)=a+g(-a+c)$, thus $j(-a+c)=g(-a+c)$ and $(g-j)(-a+c)=0$. We conclude that

$$
\begin{aligned}
(u, a) & \longrightarrow(u, a+(-a+c)) \\
& \longrightarrow \cdots \\
& \longrightarrow(u, a+(g-j)(-a+c))=(u, a) .
\end{aligned}
$$

Lemma 2.2. For each $v \in V(D)$ and $a, b \in G$, there is a digraph automorphism $f$ on $D_{\times}$with $f(v, a)=(v, b)$. In particular, the strong components of $D_{\times}$are isomorphic. 
Proof. Let $f: V(D) \times G \rightarrow V(D) \times G$ be defined $f(v, c)=(v, b-a+c)$. We have $f(v, a)=(v, b)$, and clearly $f$ is a bijection. Take an arc from $(u, c)$ to $(w, d)$. Then $c+w_{\sigma}=d$, so $b-a+c+w_{\sigma}=b-a+d$, so there is also an arc from $f(u, c)$ to $f(w, d)$. This automorphism is mentioned in [GT87, §2.2.1].

The second claim follows since every strong component contains a vertex $(v, c)$ for some $c \in G$, which follows from the strong connectedness of $D$.

Aperiodicity of $D_{\times}$does not follow from aperiodicity of $D$, as shown in Example 2.2, so it must be verified separately.

Example 2.2. The condition of aperiodicity of $D_{\times}$cannot be transfered from $D$. For example, if $a \in G$ has order at least 3 and if $E(D)=\{(a, a)\}$ then $D_{\times}$is periodic. Figure 6 shows a less trivial counterexample digraph $D$.

Notation 2.5. For real sequences $f(n), g(n)$, the notation $f(n)=O(g(n))$ means there is $c>0$ such that for all sufficiently large $n$ we have $|f(n)| \leq c g(n)$. The notation $f(n)=\Theta(g(n))$ means there are $c, d>0$ such that for all sufficiently large $n$ we have $|f(n)| \leq c g(n)$ and $|f(n)| \geq d g(n)$.

The following basic result applies the Perron-Frobenius theorem to asymptotic counting.

Proposition 2.2. Let $M$ be a nonzero $n \times n$ adjacency matrix of a strongly connected and aperiodic digraph. Then if $\alpha, \beta \in \mathbb{R}^{n}$, we have

$$
\alpha^{\top} M^{m} \beta=\left(\alpha \cdot v_{\lambda}\right)\left(u_{\lambda} \cdot \beta\right) \lambda^{m}\left(1+O\left(\theta^{m}\right)\right), \quad m \rightarrow \infty,
$$

where $\lambda \geq 1$ is the largest-magnitude eigenvalue of $M, v_{\lambda}$ is a positive $\lambda$-eigenvector 


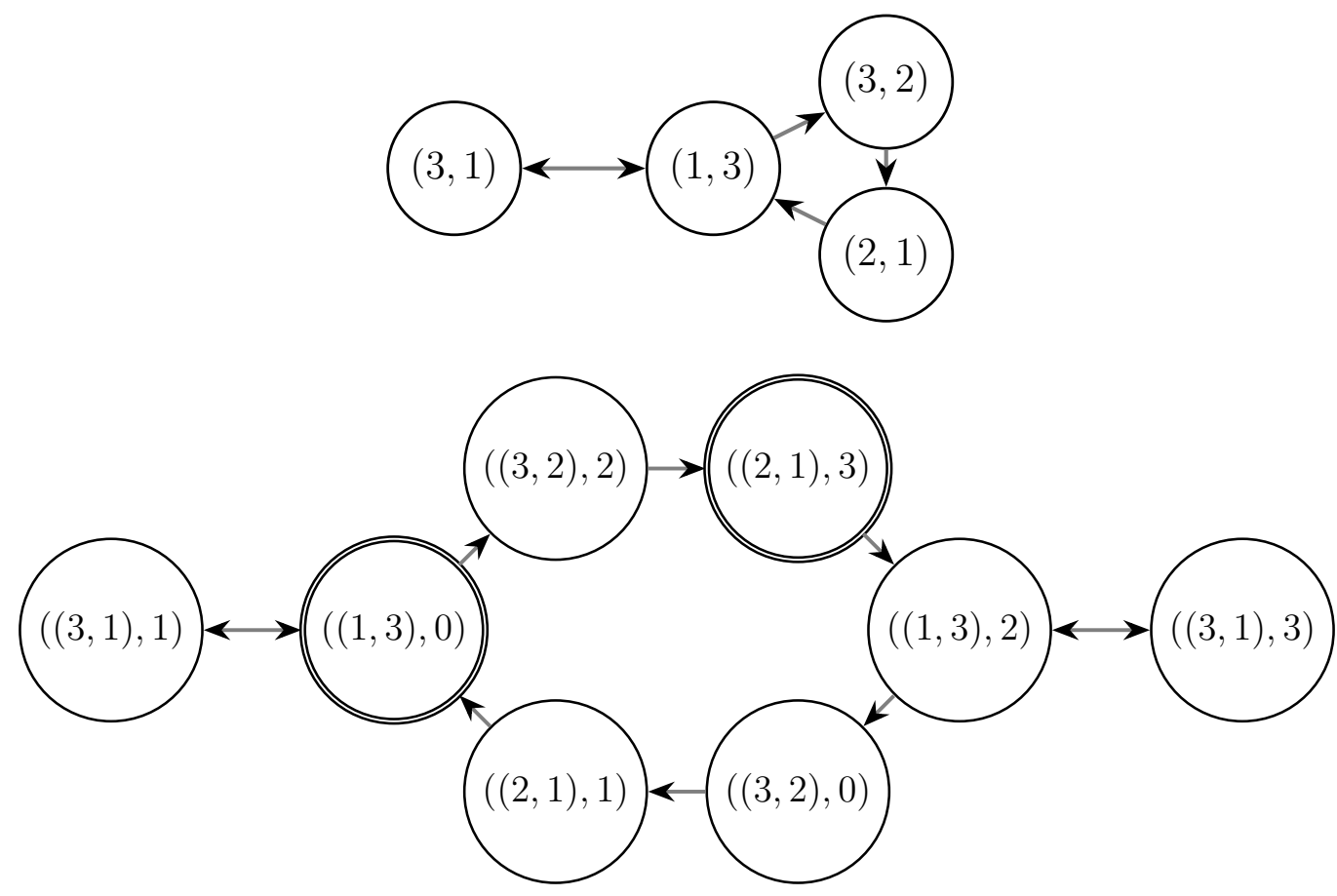

Figure 6: An aperiodic strongly connected digraph $D$ (above) with vertices in $\mathbb{Z}_{4}^{2}$ such that $D_{\times}$(one component shown below) has period 2. Examples with connected $D_{\times}$ exist as well, such as the above $D$ over $\mathbb{Z}_{8}$ with 7 replacing 3 and 3 replacing 2 . 
of $M, u_{\lambda}$ is a positive $\lambda$-eigenvector of $M^{\top}$ such that $v_{\lambda} \cdot u_{\lambda}=1$, and $0 \leq \theta<1$.

Proof. By [Sch74, Proposition 2.4], any largest-magnitude eigenvalue $\lambda$ of $M$ satisfies $|\lambda| \geq 1$. We use a few other facts from linear algebra covered in e.g. [Mey00; Zan17]. By the Perron-Frobenius theorem, we conclude there is a unique largest-magnitude eigenvalue $\lambda>0$ and $\lambda$ has multiplicity 1 . Furthermore, $M$ has Jordan decomposition

$$
M=\left[\begin{array}{cc}
\mid & \\
v_{\lambda} & * \\
\mid &
\end{array}\right]\left[\begin{array}{l|l}
\lambda & \mathbf{0} \\
\hline \mathbf{0} & B
\end{array}\right]\left[\begin{array}{cc}
- & u_{\lambda}- \\
& *
\end{array}\right],
$$

where $v_{\lambda}$ is a positive $\lambda$-eigenvector of $M, u_{\lambda}$ is a positive $\lambda$-eigenvector of $M^{\top}$, and $B$ is a block-diagonal matrix with spectral radius $0 \leq r<\lambda$. The fact that $v_{\lambda} \cdot u_{\lambda}=1$ follows once we note that the first and last matrices in a Jordan decomposition are inverses. Taking powers, we have

$$
M^{m}=\left[\begin{array}{c}
\mid \\
v_{\lambda}
\end{array}\right]\left[\begin{array}{c|c}
\lambda^{m} & \mathbf{0} \\
\hline \mid &
\end{array}\right]\left[\begin{array}{cc}
- & u_{\lambda}- \\
& *
\end{array}\right],
$$

where entries of $B^{m}$ are $O\left(r^{m}\right)$. The result is now immediate with $\theta=r / \lambda$.

The essential idea of Proposition 2.2 is quite classical, see e.g. [FS09, Corollary V.1].

It is sometimes useful to know more about the growth rate of the number of walks.

Proposition 2.3. Let $M$ be an $n \times n$ adjacency matrix of a strongly connected and aperiodic digraph. If $n \geq 2$ then all entries of $M^{m}$ are $\Theta\left(B^{m}\right)$, where $B>1$. 
Proof. Let $v_{1}$ be a vertex in the digraph. Since the digraph is aperiodic, there are two distinct cycles $C_{1}$ and $C_{2}$ starting from $v_{1}$; let their lengths be $c_{1}, c_{2}$. Let $\ell=$ $\operatorname{lcm}\left(c_{1}, c_{2}\right)$. Construct a walk of length $\alpha \ell$ by choosing $\alpha$ segments of length $\ell$ where each segment is either $C_{1}$ repeated or $C_{2}$ repeated. Then the number of walks of length $\alpha \ell$ is at least $\left(2^{1 / \ell}\right)^{\alpha \ell}$. By Proposition 2.2 the number of walks of length $m$ is $\Theta\left(B^{m}\right)$ so we must have $B>1$.

Definition 2.3. A de Bruijn subgraph $D$ is regular if $D$ is strongly connected, contains at least 2 vertices, and its derived digraph $D_{\times}$is aperiodic.

Notation 2.6. If two real sequences satisfy $\lim _{n \rightarrow \infty} f(n) / g(n)=1$, we write $f(n) \sim$ $g(n)$ and say $f(n)$ is asymptotic to $g(n)$.

Proposition 2.4. Suppose $D$ is regular and $p(m ; D, \Psi, \Phi) \sim A \cdot B^{m}$. Then either $p_{s}(m ; D, \Psi, \Phi)=0$ or

$$
p_{s}(m ; D, \Psi, \Phi)=C_{s} \cdot B^{m}\left(1+O\left(\theta^{m}\right)\right), \quad m \rightarrow \infty
$$

where $C_{s}>0$ can be computed from $D_{\times}$and $0 \leq \theta<1$.

Proof. Since the strong components of $D_{\times}$are isomorphic by Lemma 2.1 and Lemma 2.2, they each have the same adjacency matrix and the same eigenvalues. The only difference between a composition of $s$ and an arbitrary composition is the allowed finish vertices. Thus by Proposition 2.1 and Proposition 2.2 we conclude $p_{s}(m ; D, \Psi, \Phi)=C_{s} \cdot B^{m}\left(1+O\left(\theta^{m}\right)\right)$ where $C_{s}=0$ only if $p_{s}(m ; D, \Psi, \Phi)=0$. The latter case occurs if there is no strong component of $D_{\times}$containing vertices from both $\Psi_{\times}$and $\Phi_{s}$.

The asymptotics of $p_{s}(m ; D, \Psi, \Phi)$ are now established. However, in some cases we 
can usefully simplify the constants involved.

Definition 2.4. If $A$ is an $m \times n$ matrix and $B$ is a $p \times q$ matrix, then the Kronecker product $A \otimes B$ is the $m p \times n q$ matrix $C$ such that $[C]_{p(r-1)+v, q(s-1)+w}=[A]_{r, s}[B]_{v, w}$. Visually,

$$
A \otimes B=\left[\begin{array}{ccc}
{[A]_{1,1} B} & \cdots & {[A]_{1, n} B} \\
\vdots & \ddots & \vdots \\
{[A]_{m, 1} B} & \cdots & {[A]_{m, n} B}
\end{array}\right] .
$$

Basic properties of the Kronecker product are discussed in [HJ94, Chapter 4]. We quote a couple of relevant facts.

Proposition 2.5 (Lemma 4.2.10 in [HJ94]). Let $F$ be a field. Let $A \in F^{m \times n}, B \in$ $F^{p \times q}, C \in F^{n \times k}, D \in F^{q \times r}$. Then $(A \otimes B)(C \otimes D)=A C \otimes B D$.

Proposition 2.6 (Equation 4.2.8 in [HJ94]). Let $F$ be a field. We have

$$
A \otimes(B+C)=A \otimes B+A \otimes C
$$

for all $A \in F^{m \times n}$ and $B, C \in F^{p \times q}$.

Notation 2.7. For a logical statement $\phi$, the notation $[\phi]$ stands for 1 if $\phi$ is true and 0 otherwise.

We next establish the structure of derived digraphs $D_{\times}$in terms of the Kronecker product.

Lemma 2.3. Say $|V(D)|=\alpha$ and fix a vertex ordering $v_{1}, \ldots, v_{\alpha}$. Let $M$ be the adjacency matrix of $D$ with respect to this ordering. Also fix an ordering on $G=$ $\left\{a_{1}, a_{2}, \ldots, a_{\beta}\right\}$ where $a_{1}=0$. Finally, define a vertex ordering on $D_{\times}$as

$$
\left(v_{1}, a_{1}\right), \ldots,\left(v_{\alpha}, a_{1}\right),\left(v_{1}, a_{2}\right), \ldots,\left(v_{\alpha}, a_{2}\right), \ldots,\left(v_{1}, a_{\beta}\right), \ldots,\left(v_{\alpha}, a_{\beta}\right)
$$


Let $M_{\times}$be the adjacency matrix of $D_{\times}$with respect to this ordering. For each $a \in G$, define the $\alpha \times \alpha$ matrix $M_{a}$ and $\beta \times \beta$ matrix $P_{a}$ such that

$$
\begin{aligned}
{\left[M_{a}\right]_{i, j} } & =\left[v_{j}(\sigma)=a,\left(v_{i}, v_{j}\right) \in E(D)\right], \\
{\left[P_{a}\right]_{i, j} } & =\left[a_{i}+a=a_{j}\right] .
\end{aligned}
$$

Then $M_{\times}=\sum_{a \in G} P_{a} \otimes M_{a}$ and $M=\sum_{a \in G} M_{a}$.

Proof. We have

$$
\left[M_{\times}\right]_{i+\alpha(j-1), k+\alpha(l-1)}=\left[\left(v_{i}, v_{k}\right) \in E(D), a_{j}+v_{k}(\sigma)=a_{l}\right]
$$

and

$$
\begin{aligned}
{\left[\sum_{a \in G} P_{a} \otimes M_{a}\right]_{i+\alpha(j-1), k+\alpha(l-1)} } & =\sum_{a \in G}\left[P_{a}\right]_{j, l}\left[M_{a}\right]_{i, k} \\
& =\sum_{a \in G}\left[a_{j}+a=a_{l}\right]\left[v_{k}(\sigma)=a,\left(v_{i}, v_{k}\right) \in E(D)\right] \\
& =\left[\left(v_{i}, v_{k}\right) \in E(D), a_{j}+v_{k}(\sigma)=a_{l}\right] .
\end{aligned}
$$

Clearly

$$
[M]_{i, j}=\left[\left(v_{i}, v_{j}\right) \in E(D)\right]=\sum_{a \in G}\left[v_{j}(\sigma)=a,\left(v_{i}, v_{j}\right) \in E(D)\right]=\sum_{a \in G}\left[M_{a}\right]_{i, j} .
$$

Theorem 2.1. Assume

- for some $v \in V(D)$ we have that for all $a \in G$ there is a legal composition starting and ending with $v$ with total $a$, and 
- for some $u \in V(D)$ the set

$$
\left\{m: \exists a \text { walk } x=(u, v, \ldots, w, u) \text { of length } m+1, \sum x=\sum u\right\}
$$

has a GCD of 1 , where $\sum x$ is the total of the composition corresponding to $x$.

Assume $p(m ; D, \Psi, \Phi) \sim A \cdot B^{m}$. Then

$$
p_{a}(m ; D, \Psi, \Phi)=\frac{A}{|G|} \cdot B^{m}\left(1+O\left(\theta^{m}\right)\right), \quad m \rightarrow \infty, 0 \leq \theta<1 .
$$

Proof. From the first condition we know there is a single strong component by Lemma 2.1, i.e. $D_{\times}$is strongly connected. The second condition ensures that this component is aperiodic. This allows us to conclude that the Perron-Frobenius theorem applies directly to $D_{\times}$.

Let $M, M_{\times}, M_{a}, P_{a}$ be as in Lemma 2.3 .

Let $\lambda>0$ be the dominant eigenvalue of $M$, let $v_{\lambda}$ be an associated positive eigenvector, and let $u_{\lambda}$ be an associated positive left eigenvector (eigenvector of $M^{\top}$ ). Let $\xi \in \mathbb{R}^{\beta}$ be the all-1 vector $\left[\begin{array}{llll}1 & 1 & \cdots & 1\end{array}\right]$.

We claim that $\xi \otimes v_{\lambda}$ is an eigenvector of $M_{\times}$with eigenvalue $\lambda$. First, by Proposition 2.5, $\left(P_{a} \otimes M_{a}\right)\left(\xi \otimes v_{\lambda}\right)=P_{a} \xi \otimes M_{a} v_{\lambda}$. Since $P_{a}$ is a permutation matrix, we have 
$P_{a} \xi=\xi$. Thus

$$
\begin{aligned}
M_{\times}\left(\xi \otimes v_{\lambda}\right) & =\left(\sum_{a \in G} P_{a} \otimes M_{a}\right)\left(\xi \otimes v_{\lambda}\right) \\
& =\sum_{a \in G}\left(P_{a} \otimes M_{a}\right)\left(\xi \otimes v_{\lambda}\right) \\
& =\sum_{a \in G} P_{a} \xi \otimes M_{a} v_{\lambda} \\
& =\sum_{a \in G} \xi \otimes M_{a} v_{\lambda} .
\end{aligned}
$$

By Proposition 2.6, $\sum_{a \in G} \xi \otimes M_{a} v_{\lambda}=\xi \otimes \sum_{a \in G} M_{a} v_{\lambda}$. We conclude

$$
\begin{aligned}
M_{\times}\left(\xi \otimes v_{\lambda}\right) & =\xi \otimes \sum_{a \in G} M_{a} v_{\lambda} \\
& =\xi \otimes\left(\sum_{a \in G} M_{a}\right) v_{\lambda} \\
& =\xi \otimes M v_{\lambda} \\
& =\xi \otimes \lambda v_{\lambda} \\
& =\lambda \xi \otimes v_{\lambda} .
\end{aligned}
$$

Similarly we have that $\xi \otimes u_{\lambda}$ is a left eigenvector for $M_{\times}$with eigenvalue $\lambda$.

By Proposition 2.2, we know that

$$
p_{a}(m ; D, \Psi, \Phi)=\psi_{\times}^{\top} M_{\times}^{m-\sigma} \phi_{a}=C_{a} \lambda^{m}\left(1+O\left(\theta^{m}\right)\right)
$$

where $C_{a}=c\left(\psi_{\times} \cdot\left(\xi \otimes v_{\lambda}\right)\right)\left(\left(\xi \otimes u_{\lambda}\right) \cdot \phi_{a}\right)$ for some fixed scaling factor $c>0$.

Suppose $a=a_{i}$; then $\phi_{a}=e_{i} \otimes \phi$. Thus

$$
\left(\xi \otimes u_{\lambda}\right) \cdot \phi_{a}=\left(\xi \otimes u_{\lambda}\right) \cdot\left(e_{i} \otimes \phi\right)=\left(\xi \cdot e_{i}\right) \otimes\left(u_{\lambda} \cdot \phi\right)=u_{\lambda} \cdot \phi .
$$

Since $u_{\lambda} \cdot \phi$ does not depend on $a$, the proof is now complete. 
An alternative proof approach involves using Lemma 2.2 and the fact that automorphisms of $M_{\times}$correspond to permutation matrices $P$ such that $P M_{\times} P^{-1}=M_{\times}$.

Corollary 2.1. Assume the conditions of Theorem 2.1. Construct a probability space from $\mathcal{P}(m ; D, \Psi, \Phi)$ and the uniform probability measure. Then for $a \in G$, let $\mathbb{P}_{m}(a)$ be the probability that an element drawn randomly from $\mathcal{P}(m ; D, \Psi, \Phi)$ has total a. We have for any $a \in G$

$$
\mathbb{P}_{m}(a) \rightarrow \frac{1}{|G|}, \quad m \rightarrow \infty
$$

or in other words, $\mathbb{P}_{m}$ converges strongly to the uniform measure on $G$.

Proof. Direct from Theorem 2.1.

Example 2.3. We show a case where the strong connectedness condition in Theorem 2.1 is required. Let $D$ be the digraph given in Figure 7 , where $G=\mathbb{Z}_{2}$. Assume $\Psi=\Phi=V(D)$.

Let $M^{\langle 1\rangle}, M^{\langle 2\rangle}$ be adjacency matrices of the two strong components of $D_{\times}$, under a particular vertex ordering. We have

$$
M^{\langle 1\rangle}=M^{\langle 2\rangle}=\left[\begin{array}{llllll}
1 & 1 & 0 & 0 & 0 & 0 \\
0 & 0 & 1 & 0 & 0 & 0 \\
0 & 0 & 0 & 1 & 0 & 0 \\
0 & 0 & 0 & 0 & 1 & 0 \\
0 & 0 & 0 & 0 & 0 & 1 \\
1 & 0 & 0 & 0 & 0 & 0
\end{array}\right] .
$$

From a Jordan decomposition, we get a left eigenvalue

$$
u_{\lambda} \doteq\left[\begin{array}{llllll}
0.368841 & 0.286991 & 0.223305 & 0.173751 & 0.135194 & 0.105193
\end{array}\right]
$$



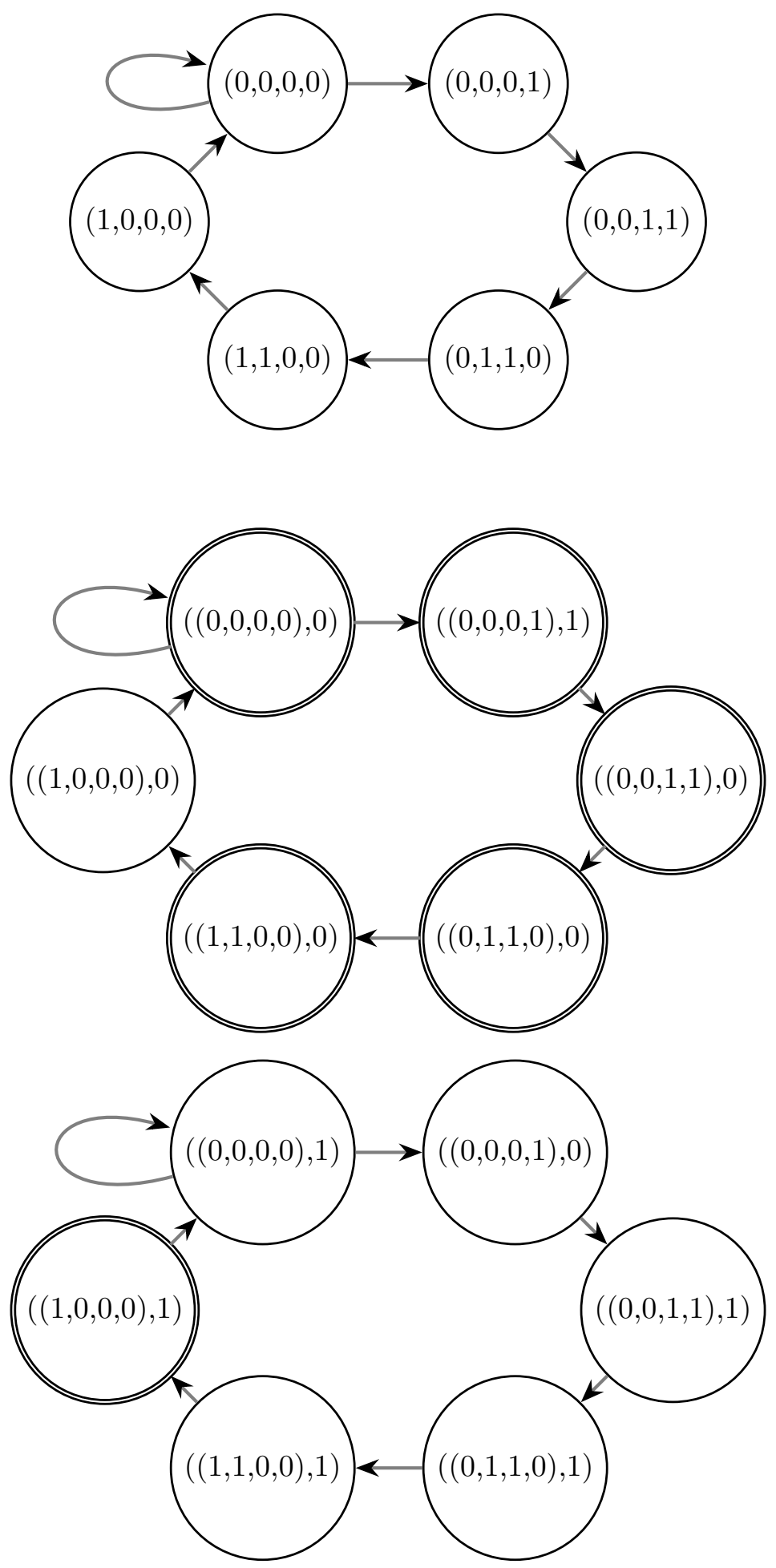

Figure 7: A base digraph $D$ (above) and derived digraph $D_{\times}$with 2 strong components (below). The vertices of $D$ are 4 -tuples over $\mathbb{Z}_{2}$. 
and a right eigenvector

$$
v_{\lambda} \doteq\left[\begin{array}{llllll}
1.2852 & 0.366538 & 0.471074 & 0.605423 & 0.77809 & 1.0
\end{array}\right]
$$

corresponding to the dominant eigenvalue $\lambda \doteq 1.2852$. Also

$$
\begin{gathered}
\psi_{\times}^{\langle 1\rangle}=\left[\begin{array}{llllll}
1 & 1 & 1 & 1 & 1 & 0
\end{array}\right]^{\top}, \phi_{0}^{\langle 1\rangle}=\left[\begin{array}{llllll}
1 & 0 & 1 & 1 & 1 & 1
\end{array}\right]^{\top}, \\
\phi_{1}^{\langle 1\rangle}=\left[\begin{array}{llllll}
0 & 1 & 0 & 0 & 0 & 0
\end{array}\right]^{\top},
\end{gathered}
$$

and

$$
\begin{gathered}
\psi_{\times}^{\langle 2\rangle}=\left[\begin{array}{llllll}
0 & 0 & 0 & 0 & 0 & 1
\end{array}\right]^{\top}, \phi_{0}^{\langle 2\rangle}=\left[\begin{array}{llllll}
0 & 1 & 0 & 0 & 0 & 0
\end{array}\right]^{\top}, \\
\phi_{1}^{\langle 2\rangle}=\left[\begin{array}{llllll}
1 & 0 & 1 & 1 & 1 & 1
\end{array}\right]^{\top} .
\end{gathered}
$$

We compute

$$
\begin{aligned}
p_{0}(m ; D) & \left.=\psi_{\times}^{\langle 1\rangle^{\top}}\left(M^{\langle 1\rangle}\right)^{m-4} \phi_{0}^{\langle 1\rangle}+\psi_{\times}^{\langle 2\rangle}\right\rangle^{\top}\left(M^{\langle 2\rangle}\right)^{m-4} \phi_{0}^{\langle 2\rangle} \\
& \sim \lambda^{m-4}\left(\left(\psi_{\times}^{\langle 1\rangle} \cdot v_{\lambda}\right)\left(u_{\lambda} \cdot \phi_{0}^{\langle 1\rangle}\right)+\left(\psi_{\times}^{\langle 2\rangle} \cdot v_{\lambda}\right)\left(u_{\lambda} \cdot \phi_{0}^{\langle 2\rangle}\right)\right) \\
& \doteq \lambda^{m-4}(3.50632 \cdot 1.00628+1.0 \cdot 0.286991) \\
& =3.81533 \cdot \lambda^{m-4}
\end{aligned}
$$

and

$$
\begin{aligned}
p_{1}(m ; D) & =\psi_{\times}^{\langle 1\rangle^{\top}}\left(M^{\langle 1\rangle}\right)^{m-4} \phi_{1}^{\langle 1\rangle}+\psi_{\times}^{\langle 2\rangle^{\top}}\left(M^{\langle 2\rangle}\right)^{m-4} \phi_{1}^{\langle 2\rangle} \\
& \sim \lambda^{m-4}\left(\left(\psi_{\times}^{\langle 1\rangle} \cdot v_{\lambda}\right)\left(u_{\lambda} \cdot \phi_{1}^{\langle 1\rangle}\right)+\left(\psi_{\times}^{\langle 2\rangle} \cdot v_{\lambda}\right)\left(u_{\lambda} \cdot \phi_{1}^{\langle 2\rangle}\right)\right) \\
& \doteq \lambda^{m-4}(3.50632 \cdot 0.286991+1.0 \cdot 1.00628) \\
& =2.01256 \cdot \lambda^{m-4} .
\end{aligned}
$$

So indeed the conclusion of Theorem 2.1 does not hold.

Lemma 2.4. Assume $G$ is abelian. Define a $D_{\times}$-automorphism $f$ by $f(v, b)=$ 


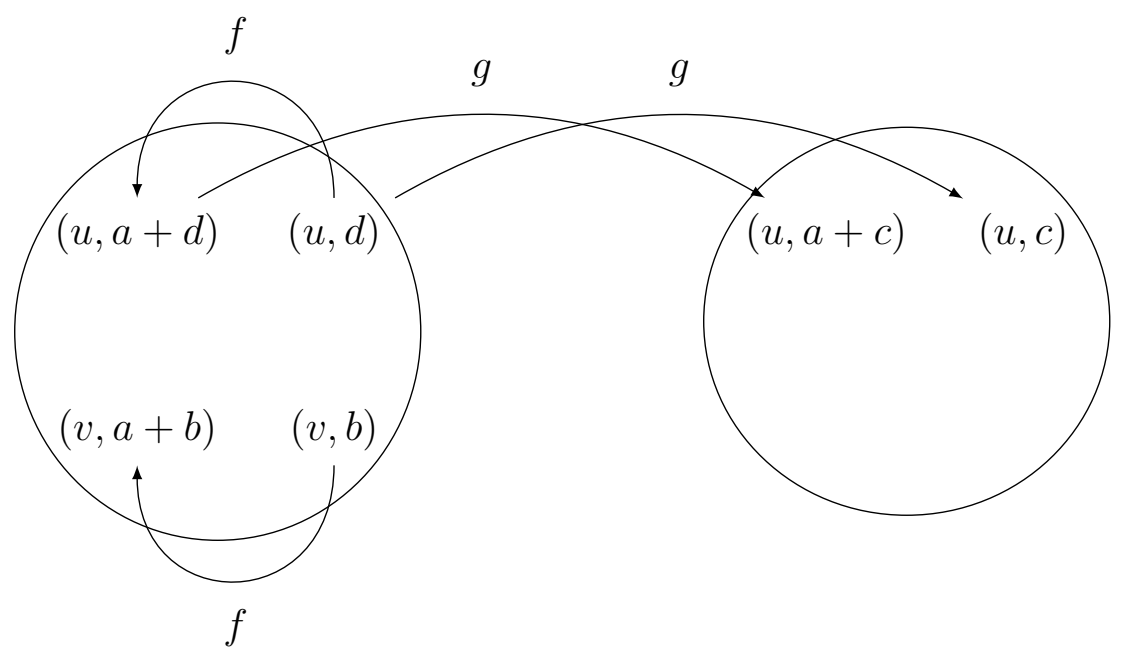

Figure 8: Strong components in the proof of Lemma 2.4.

$(v, a+b)$ for some $a \in G$. If $f$ maps any vertex to its own strong component, then $f$ maps all vertices to their own strong component.

Proof. Let $(u, c),(v, b)$ be arbitrary vertices and say $(v, b) \longrightarrow(v, a+b)$ in $D_{\times}$. We seek to show that $(u, c) \longrightarrow(u, a+c)$.

There is some $(u, d)$ in the same strong component as $(v, b)$ and so $(u, d) \longrightarrow(u, a+d)$.

The automorphism $g(w, r)=(w, c-d+r)$ maps $(u, d)$ to $(u, c)$ and $(u, a+d)$ to $(u, c-d+a+d)=(u, a+c)$.

Thus $(u, d) \longrightarrow(u, a+d)$ implies $g(u, d)=(u, c) \longrightarrow g(u, a+d)=(u, a+c)$. This is illustrated in Figure 8.

The following is a useful characterization of strong connectedness of $D_{\times}$for abelian $G$

Lemma 2.5. Assume $G$ is abelian. Let $A$ be a generating set for $G$, i.e. $\langle A\rangle=G$. If 
for all $a_{i} \in A$ there is a vertex $(v, 0) \in V\left(D_{\times}\right)$such that $(v, 0) \longrightarrow\left(v, a_{i}\right)$ in $D_{\times}$, then $D_{\times}$is strongly connected.

Proof. We show that for any $u \in V(D)$ and $r \in G$, the vertices $(u, 0)$ and $(u, r)$ are in the same strong component of $D_{\times}$.

Say $r=j(1) a_{1}+\cdots+j(p) a_{p}$ for $a_{i} \in A, j(i) \in \mathbb{Z}_{\geq 0}$. We know from Lemma 2.4 that the $D_{\times}$-automorphism $f_{j}(v, s)=\left(v, a_{j}+s\right)$ maps strong components to themselves. Thus the composition $f_{r}=f_{1}^{j(1)} \circ \cdots \circ f_{p}^{j(p)}$ also maps strong components to themselves. We conclude that $(u, 0)$ and $f_{r}(u, 0)=\left(u, j(1) a_{1}+\cdots+j(p) a_{p}\right)=(u, r)$ belong to the same strong component.

We now consider some examples of $D$.

We generalize Carlitz compositions as follows. A sequence $x \in \operatorname{SEQ}_{m}(G)$ is a $d$-Carlitz composition if every subword $x(i), \ldots, x(i+d)$ contains no repeated part. Thus Carlitz compositions are 1-Carlitz. We note that unlike for integer compositions, we generally allow the identity element as a part. We also note that this definition is consistent with [GMW18] but different from Definition 4.33 in [HM10, p. 115]. Words with no equal adjacent letters are also called Smirnov words as in [FS09, Example III.24].

Lemma 2.6. There is a de Bruijn subgraph $D$ with span $\sigma=d+1$ representing d-Carlitz compositions such that $D_{\times}$is strongly connected and aperiodic, provided $|G| \geq d+2$.

Proof. Take as vertex set for $D$ all $(d+1)$-tuples of distinct elements of $G$. The allowed start and finish vertices are all of $V(D)$. The strong connectedness of $D$ is established in (the proof of) [GMW18, Corollary 2]; we include the argument here 
for completeness. Let $u, v \in V(D)$ be distinct. Let $w$ be a vertex such that $w=$ $(w(1), \ldots, w(j), v(j+1), \ldots, v(d+1))$, and assume $u \longrightarrow w$. Clearly this is possible if $j=d+1$. If this is true for some $j \leq d+1$ we seek to show that there is a vertex $y=(y(1), \ldots, y(j-1), v(j), \ldots, v(d+1))$ such that $w \longrightarrow y$ and thus $u \longrightarrow y$. If $v(j) \notin\{w(1), \ldots, w(j)\}$ then $w \longrightarrow y=(w(1), \ldots, w(j-1), v(j), \ldots, v(d+1))$. If $v(j) \in\{w(1), \ldots, w(j)\}$, assume $w(r)=v(j)$. Let $a \in G$ be an element not found in $\{w(1), \ldots, w(j), v(j), \ldots, v(d+1)\}$. Then $w \longrightarrow(w(1), \ldots, w(r-1), a, w(r+$ $1), \ldots, w(j), v(j+1), \ldots, v(d+1)) \longrightarrow y=(w(1), \ldots, w(r-1), a, w(r+1), \ldots, w(j-$ 1), $v(j), \ldots, v(d+1))$. By induction, we conclude that $u \longrightarrow w$ in the case $j=0$, i.e. $u \longrightarrow v$.

To show strong connectedness of $D_{\times}$, we fix a vertex $\left(a_{1}, \ldots, a_{d+1}\right) \in V(D)$ and for any $s \in G$ exhibit a walk from $\left(\left(a_{1}, \ldots, a_{d+1}\right), 0\right)$ to $\left(\left(a_{1}, \ldots, a_{d+1}\right), s\right)$. Let $n$ be the order of $\Sigma a=a_{1}+\cdots+a_{d+1}$. We consider two cases.

Case 1: $s \notin\left\{a_{1}, \ldots, a_{d+1}\right\}$. The first step is to $\left(a_{2}, \ldots, a_{d+1}, s\right)$. Follow this by the $(d+1)$-step path to $\left(a_{1}, \ldots, a_{d+1}\right)$ Take the $(d+1)$-step path back to $\left(a_{1}, \ldots, a_{d+1}\right)$ exactly $n-1$ times. The total of this walk is

$$
s+\Sigma a+(n-1) \Sigma a=s,
$$

thus $\left(\left(a_{1}, \ldots, a_{d+1}\right), 0\right) \longrightarrow\left(\left(a_{1}, \ldots, a_{d+1}\right), s\right)$ in $D_{\times}$.

Case 2: $s=a_{j}$ for some $1 \leq j \leq d+1$. Let $b$ represent some element of $G$ not in $\left\{a_{1}, \ldots, a_{d+1}\right\}$. Begin with the $(d+1)$-step path to $\left(b, a_{1}, \ldots, a_{j-1}, a_{j+1}, a_{d+1}\right)$. Let $n_{1}$ be the order of $b+a_{1}+\cdots+a_{j-1}+a_{j+1}+a_{d+1}$. Follow the $(d+1)$ step path back to $\left(b, a_{1}, \ldots, a_{j-1}, a_{j+1}, a_{d+1}\right)$ exactly $n_{1}-1$ times. Traverse one arc to $\left(a_{1}, \ldots, a_{j-1}, a_{j+1}, a_{d+1}, s\right)$, then follow the $(d+1)$-step path to 
$\left(a_{1}, \ldots, a_{j-1}, a_{j+1}, a_{d+1}, b\right)$. Let $n_{2}$ be the order of $a_{1}+\ldots+a_{j-1}+a_{j+1}+a_{d+1}+b$. Take the $(d+1)$-step path back to $\left(a_{1}, \ldots, a_{j-1}, a_{j+1}, a_{d+1}, b\right)$ exactly $n_{2}-1$ times. Finally take the $(d+1)$-step path to $\left(a_{1}, \ldots, a_{d+1}\right)$ and cycle $\left(a_{1}, \ldots, a_{d+1}\right)$ the suitable number of times. The total of this walk is $0+s+0+0=s$ so $\left(\left(a_{1}, \ldots, a_{d+1}\right), 0\right) \longrightarrow\left(\left(a_{1}, \ldots, a_{d+1}\right), s\right)$ in $D_{\times}$.

We now turn to aperiodicity. Take a $D$-vertex $u=\left(a_{1}, \ldots, a_{d+1}\right)$ that does not contain the part 0 . We give two closed walks starting from $u$ with total 0 and lengths that differ by 1 .

Let $n$ be the order of $a_{1}+\cdots+a_{d+1}$. The first walk repeats the $(d+1)$-step cycle back to $u$ exactly $n$ times. The second walk first takes a step to $\left(u_{2}, \ldots, u_{d+1}, 0\right)$ followed by the $(d+1)$-step path to $u$. Then we cycle back to $u$ exactly $n-1$ times.

Notation 2.8. We denote the falling factorial $n(n-1) \cdots(n-k+1)$ by $n^{\underline{k}}$.

Proposition 2.7. The number of d-Carlitz m-compositions of $s \in G$ over a finite group $G$ is

$$
\frac{1}{|G|}|G|^{\underline{d}}(|G|-d)^{m-d}\left(1+O\left(\theta^{m}\right)\right), \quad m \rightarrow \infty, 0 \leq \theta<1,
$$

provided $|G| \geq d+2$.

Proof. With Lemma 2.6 we conclude that the conditions of Theorem 2.1 are satisfied.

In $D$ each vertex has an out-degree of $|G|-d$. This allows us to count walks in $D$ directly. We have $V(D)=|G| \underline{d+1}$. Thus the number of $m$-compositions represented by $D$ is $|G| \stackrel{d+1}{=}(|G|-d)^{m-d-1}=|G| \underline{d}(|G|-d)^{m-d}$. We conclude by applying Theorem 2.1 . 


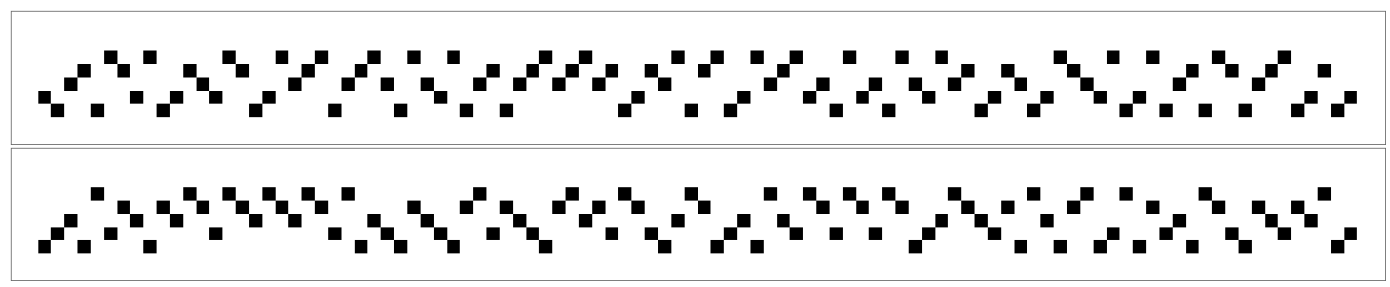

Figure 9: Uniform-randomly generated 2-Carlitz 100-compositions of 0 (above) and 1 (below) over $\mathbb{Z}_{5}$.

\begin{tabular}{|r|rrr|}
\hline$m$ & $i d$ & $(123)$ & $(12)$ \\
\hline 3 & 27 & 24 & 25 \\
4 & 134 & 128 & 120 \\
5 & 613 & 631 & 625 \\
6 & 3096 & 3102 & 3150 \\
7 & 15667 & 15604 & 15625 \\
8 & 78224 & 78263 & 78000 \\
9 & 390513 & 390681 & 390625 \\
10 & 1952696 & 1952402 & 1953750 \\
11 & 9765817 & 9765529 & 9765625 \\
12 & 48830424 & 48831663 & 48825000 \\
13 & 244140763 & 244140556 & 244140625 \\
14 & 1220690096 & 1220686202 & 1220718750 \\
15 & 6103512717 & 6103517079 & 6103515625 \\
16 & 30517650374 & 30517659188 & 30517500000 \\
\hline
\end{tabular}

Table 1: Counts of Carlitz $m$-compositions of some $a$ over $S_{3}$.

Figure 9 shows randomly generated 2-Carlitz 100-compositions over $\mathbb{Z}_{5}$. Table 1 gives counts for Carlitz $m$-compositions of $a$ over $S_{3}$.

As in [GMW18] we say an $m$-composition $x$ is locally $d$-Mullen if no nonempty subword of $x$ of length at most $d$ has total 0 .

Proposition 2.8. The number of locally $d$-Mullen $m$-compositions of $a \in G$ over a finite group $G$ is

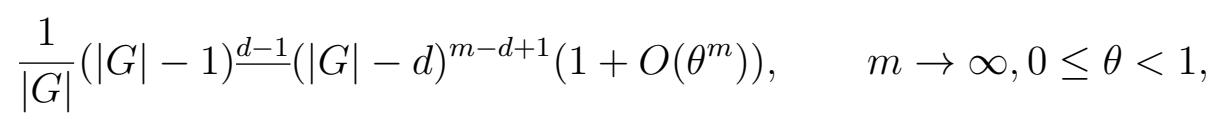

provided $|G| \geq d+2$. 
Proof. Let $D^{\langle 1\rangle}$ be the digraph of span $d$ representing locally $(d+1)$-Mullen compositions, and let $D_{\times}^{\langle 1\rangle}$ be the derived digraph. Let $D^{\langle 2\rangle}$ be the digraph of span $d+1$ representing $d$-Carlitz compositions. We note that $\left|V\left(D_{\times}^{\langle 1\rangle}\right)\right|=\left|V\left(D^{\langle 2\rangle}\right)\right|=|G| \underline{d+1}$, recalling that the part 0 is never allowed in a locally Mullen composition. Define a function $f: V\left(D_{\times}^{\langle 1\rangle}\right) \rightarrow V\left(D^{\langle 2\rangle}\right)$ as follows:

$$
f\left(\left(v_{1}, \ldots, v_{d}\right), a\right)=\left(a-\sum_{j=1}^{d} v_{j}, a-\sum_{j=2}^{d} v_{j}, \ldots, a-v_{d}, a\right) .
$$

A computation gives us that $f$ is a graph isomorphism from $D_{\times}^{\langle 1\rangle}$ to $D^{\langle 2\rangle}$. Thus the strong connectedness and aperiodicity of $D^{\langle 2\rangle}$ established in Lemma 2.6 hold for $D_{\times}^{\langle 1\rangle}$ as well and Theorem 2.1 applies.

A part $x(i)$ in a locally $d$-Mullen compositions must not take the value $0,-x(i-$ $1),-x(i-1)-x(i-2)$, etc. and these values are distinct since

$$
n>n^{\prime}, \sum_{j=1}^{n}(-x(i-j))=\sum_{j=1}^{n^{\prime}}(-x(i-j)) \Longrightarrow \sum_{j=n^{\prime}+1}^{n}(-x(i-j))=0 .
$$

The number of locally $d$-Mullen $m$-compositions with any total is then $(|G|-$

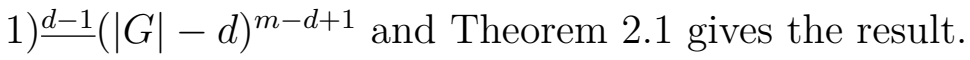

Proposition 2.9. The number of $m$-compositions of $s \in G$ over a finite group $G$ such that the sum of any $d+1$ consecutive parts is not 0 is

$$
|G|^{d-1}(|G|-1)^{m-d}\left(1+O\left(\theta^{m}\right)\right), \quad m \rightarrow \infty, 0 \leq \theta<1,
$$

provided $d \leq|G|-2$.

Proof. Define the appropriate $D$ so that $V(D)$ contains all $(d+1)$-tuples of vertices that do not sum to 0 . The strong connectedness of $D$ is established in (the proof of) [GMW18, Corollary 2]; we include the argument here for completeness. Let 
$u, v \in V(D)$ be distinct. Let $w$ be a vertex such that $w=(w(1), \ldots, w(j), v(j+$ $1), \ldots, v(d+1)$ ), and assume $u \longrightarrow w$. Clearly this is possible if $j=d+1$. If this is true for some $j \leq d+1$ we seek to show that there is a vertex $y=(y(1), \ldots, y(j-$ 1), $v(j), \ldots, v(d+1))$ such that $w \longrightarrow y$ and thus $u \longrightarrow y$. Let $a \in G$ satisfy

$$
\begin{array}{r}
w(1)+\cdots+w(j-2)+a+w(j)+v(j+1)+\cdots+v(d+1) \neq 0 \\
w(1)+\cdots+w(j-2)+a+v(j)+\cdots+v(d+1) \neq 0 .
\end{array}
$$

Then $w \longrightarrow(w(1), \ldots, w(j-2), a, w(j), v(j+1), \ldots, v(d+1)) \longrightarrow y=$ $(w(1), \ldots, w(j-2), a, v(j), \ldots, v(d+1))$. By induction, we conclude that $u \longrightarrow w$ in the case $j=0$, i.e. $u \longrightarrow v$.

We turn to strong connectedness of $D_{\times}$. Let $u=\left(a_{1}, \ldots, a_{d+1}\right)$ be an arbitrary vertex in $V(D)$, and let $s$ be an element of $G$. We seek a path (or a walk) from $(u, 0)$ to $(u, s)$ in $D_{\times}$.

Let $b \in G$ satisfy the system

$$
\begin{array}{r}
a_{1}+\cdots+a_{d}+b \neq 0 \\
a_{2}+\cdots+a_{d}+b+s \neq 0 .
\end{array}
$$

This gives at least $|G|-2$ possible values for $b$.

Let $b^{\prime} \in G$ satisfy the system

$$
\begin{gathered}
a_{j}+\cdots+a_{d}+b+s+b^{\prime}+a_{2}+\cdots+a_{j-2} \neq 0, \quad 3 \leq j \leq d+1 \\
s+b^{\prime}+a_{2}+\cdots+a_{d} \neq 0 \\
b^{\prime}+a_{2}+\cdots+a_{d+1} \neq 0 .
\end{gathered}
$$

This gives at least $|G|-d-1$ possible values for $b^{\prime}$. 


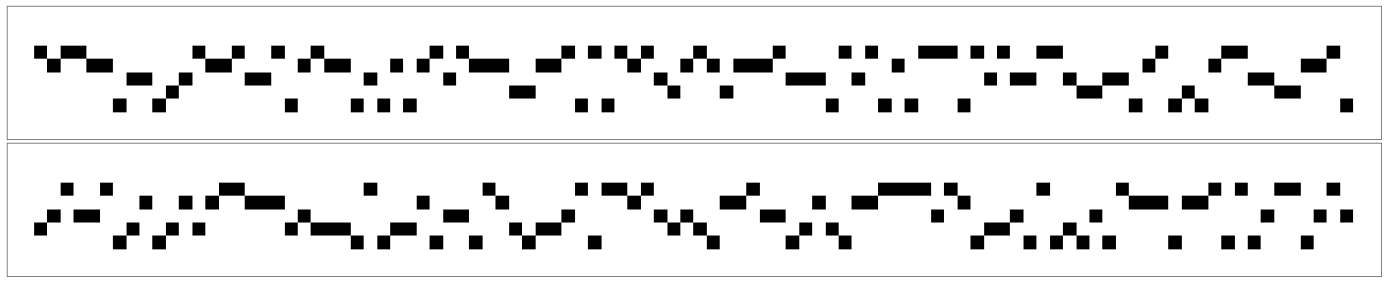

Figure 10: Uniform-randomly generated 100-compositions of 0 (above) and 1 (below) over $\mathbb{Z}_{5}$ with $x(i) \neq-x(i+1)$.

Starting from $u$, we take a $(d+1)$-step walk to $\left(a_{1}, \ldots, a_{d}, b\right)$. Let $n_{1}$ be the order of $a_{1}+\cdots+a_{d}+b$. We cycle back to $\left(a_{1}, \ldots, a_{d}, b\right)$ exactly $n_{1}-1$ times. Now we take one step by appending $s$. Then we take a $(d+1)$-step walk to $b^{\prime}, a_{2}, \ldots, a_{d+1}$ and cycle that vertex the appropriate number of times. Finally walk to and cycle $a_{1}, \ldots, a_{d+1}$. The total of this walk is $0+s+0+0=s$. We conclude that $D_{\times}$is strongly connected.

To establish aperiodicity, let $u=\left(a_{1}, \ldots, a_{d+1}\right)$ be a $D$-vertex satisfying the following. Set $a_{d}$ so that $a_{1}+\cdots+a_{d} \neq 0$. Set $a_{d+1}$ so that for $i=1, \ldots, d$ we have $\Sigma a-a_{i} \neq 0$. Thus for $i=1, \ldots, d+1$ we have $\Sigma a-a_{i} \neq 0$. There are at least $|G|-d$ possible values for $a_{d+1}$. Then we may take the same approach as in the proof of Proposition 2.7 where we consider two cycles from $u$, one with an extra 0 inserted.

We have $|V(D)|=|G|^{d}(|G|-1)$ and each vertex has out-degree $|G|-1$. Thus there are $|G|^{d}(|G|-1)(|G|-1)^{m-d-1}=|G|^{d}(|G|-1)^{m-d}$ walks in $D$ defining an $m$-composition. Applying Theorem 2.1 gives the result.

Figure 10 shows uniformly-randomly generated 100-compositions over $\mathbb{Z}_{5}$ such that no part may be followed by its (additive) inverse.

Proposition 2.10. Let $p_{a}(m)$ be the number of $m$-compositions of $a \in G$ such that the sum of any $d+1$ consecutive parts is not 0 . Then for $a \neq 0, b \neq 0$, we have 
$p_{a}(m)=p_{b}(m)$. If $m$ is not a multiple of $d+1$, then $p_{0}(m)=p_{a}(m)$.

Proof. Let $x=(x(1), \ldots, x(m))$ be an $m$-composition. Let $y(i)=\sum_{n=1}^{i} x(n)$. Clearly $x$ uniquely determines $y$ and vice versa. Also, $x$ has total $a$ if and only if $y(m)=a$.

Let $y^{\langle j\rangle}(i)=y((i-1)(d+1)+j-1)$ for $j \in[d+1]$. Then $x$ satisfies the condition if and only if each $y^{\langle j\rangle}$ is Carlitz and $y^{\langle d+1\rangle}(1) \neq 0$.

First assume $m$ is not a multiple of $d+1$, so $y(m)$ is the last part of some $y^{\langle j\rangle}, j \neq d+1$. Let $\pi: G \rightarrow G$ be defined $\pi(b)=b$ for all $b \notin\{0, a\}$, and $\pi(a)=0, \pi(0)=a$. Then if we apply $\pi$ to $y^{\langle j\rangle}$ within $y$ and take differences, we get a new $x^{\prime}$ which satisfies the condition and has total 0 . Thus $p_{0}(m)=p_{b}(m)$.

Second, if $m$ is a multiple of $d+1$, the previous $\pi$ does not work since it may change whether $y^{\langle d+1\rangle}(1) \neq 0$. However, if we take some bijective $\pi^{\prime}: G \rightarrow G$ which fixes 0 and swaps two nonzero elements $a$ and $b$, and apply it to $y^{\langle d+1\rangle}$ in $y$ we conclude $p_{a}(m)=p_{b}(m)$.

Table 2 gives counts for $m$-compositions of some $a$ over the quaternion group $Q_{3}$ such that no part may be followed by its inverse.

Example 2.4. We examine restrictions where all parts are simply required to lie in a fixed set $\Xi$. We assume without loss of generality that the subset $\Xi$ generates $G$. When working with permutation groups, the meaning of "composition" as in "integer composition" is actually the same as in "functional composition".

If $\Xi=G$ then the number of compositions of $a$ is always $|G|^{m-1}$ since the first $m-1$ parts are arbitrary and the last part is uniquely determined. However if $\Xi \subset G$ this is no longer the case. 


\begin{tabular}{|c|rrr|}
\hline$m$ & $i d$ & $(1854)(2763)$ & $(1256)(3478)$ \\
\hline 2 & 0 & 8 & 8 \\
3 & 49 & 49 & 49 \\
4 & 392 & 336 & 336 \\
5 & 2401 & 2401 & 2401 \\
6 & 16464 & 16856 & 16856 \\
7 & 117649 & 117649 & 117649 \\
8 & 825944 & 823200 & 823200 \\
9 & 5764801 & 5764801 & 5764801 \\
10 & 40336800 & 40356008 & 40356008 \\
11 & 282475249 & 282475249 & 282475249 \\
12 & 1977444392 & 1977309936 & 1977309936 \\
13 & 13841287201 & 13841287201 & 13841287201 \\
14 & 96888186864 & 96889128056 & 96889128056 \\
15 & 678223072849 & 678223072849 & 678223072849 \\
16 & 4747567274744 & 4747560686400 & 4747560686400 \\
\hline
\end{tabular}

Table 2: Counts of $m$-compositions of $a$ with no part followed by its inverse, over $Q_{8}$ (written as a subgroup of $S_{8}$ ).

The digraph $D$ with vertex set $\Xi$ is clearly strongly connected, and it is straightforward to see that $D_{\times}$is strongly connected as well.

For any cycle with final arc labeled $a$ in the Cayley graph constructed from $\Xi$, there is a cycle of equal length at $((a), 0)$ in $D_{\times}$. This implies that $D_{\times}$is aperiodic if and only if the Cayley graph is aperiodic.

One way to ensure an aperiodic Cayley graph is to include $0 \in \Xi$. In general Cayley graphs are not aperiodic e.g. only an even number of transposition permutations can equal the identity since the identity is an even permutation.

We turn to the problem of counting compositions with $r>0$ occurrences of a pattern. In preparation, we quote the fundamental fact of rational generating function asymptotics which is applied a few times in the remainder.

Theorem 2.2 (Theorem IV.9 in [FS09]). If $f(z)$ is a rational function that is analytic at 0 and has poles $\alpha_{1}, \alpha_{2}, \ldots, \alpha_{m}$, then there exist $m$ polynomials $\Pi_{j}(x)$ such that for 
sufficiently large $n$ we have $\left[z^{n}\right] f(z)=\sum_{j=1}^{m} \Pi_{j}(n) \alpha_{j}^{-n}$ where the degree of $\Pi_{j}$ is the order of the pole of $f$ at $\alpha_{j}$, minus one.

Theorem 2.3. Let $\bar{D}$ be a de Bruijn graph. Let $U \subset V(\bar{D})$ and $\Psi, \Phi \subseteq V(\bar{D})$ all be nonempty and suppose $D=\bar{D}-U$ is regular with strongly connected derived digraph $D_{\times}$

Let $\mu$ be the minimum number of occurrences of $U$ (as subwords) in a composition in $\mathcal{P}(\bar{D}, \Psi, \Phi)$ that has at least 1 occurrence of $V(D)$. Assume that for all sufficiently large values of $m$ there exist compositions in $\mathcal{P}(m ; \bar{D}, V(D), V(D))$ with exactly 1 occurrence of $U$, and that $p(m ; D, V(D), V(D)) \sim A \cdot B^{m}$.

If $r \geq \max (\mu, 1), \mu \geq 0$ then the number of $m$-compositions of $a \in G$ starting in $\Psi$ and finishing in $\Phi$ with exactly $r$ occurrences of $U$ is

$$
p_{a}(m, r ; D, \Psi, \Phi)=m^{r-\mu} A_{r, \mu} \cdot B^{m}\left(1+O\left(m^{-1}\right)\right), A_{r, \mu}>0, B>1, \quad m \rightarrow \infty .
$$

Proof. Define an occurrence segment as a composition $w$ of length at least $\sigma$ where every part in $w$ is involved in an occurrence of $U$. A detour in $\bar{D}$ is an occurrence segment $w=(w(1), \ldots, w(\ell))$ where there is an arc from $V(D)$ to $(w(1), \ldots, w(\sigma))$ and an arc from $(w(\ell-\sigma+1), \ldots, w(\ell))$ to $V(D)$. The occurrence segment $w$ gives a left semi-detour if there is an arc from $V(D)$ to $(w(1), \ldots, w(\sigma))$ and $w$ gives a right semi-detour if there is an arc from $(w(\ell-\sigma+1), \ldots, w(\ell))$ to $V(D)$.

Fix elements of $v_{\Psi} \in \Psi$ and $v_{\Phi} \in \Phi$ as start and finish segments. If $v_{\Psi} \notin U$, set $\overline{v_{\Psi}}=v_{\Psi}$, and if $v_{\Psi} \in U$, set $\overline{v_{\Psi}}$ to some right semi-detour with $v_{\Psi}$ at the beginning. If $v_{\Phi} \notin U$, set $\overline{v_{\Phi}}=v_{\Phi}$ and if $v_{\Phi} \in U$, set $\overline{v_{\Phi}}$ to a left semi-detour with $v_{\Phi}$ at the end. Fix a further sequence of detours $d_{1}, \ldots, d_{n}$ so that the total number of occurrences 
of $U$ in all (semi-)detours is $r$. For $m$ sufficiently large, an $m$-composition with $r$ occurrences of $U$ has the form

$$
x=\overline{v_{\Psi}} y_{1} d_{1} y_{2} d_{2} \cdots y_{n} d_{n} y_{n+1} \overline{v_{\Phi}}
$$

where each $y_{i}$ is a non-empty composition such that no parts of $y_{i}$ are involved in an occurrence of $U$ in $x$. We further fix $a_{1}, \ldots, a_{n+1} \in G$ such that $\Sigma y_{i}=a_{i}$ implies $\Sigma x=a$. Let the total length of the $y_{i}$ be $m-\delta$.

Given all of the fixed objects, the $y_{i}$ are subject to start and finish constraints, totals, and a total length $m-\delta$. Let $c^{\langle i\rangle}(z)$ be the generating function counting possible $y_{i}$ where $z$ marks length. Then

$$
c^{\langle i\rangle}(z)=z^{\sigma} \psi_{i}^{\top}\left(\sum_{j \geq 0} M_{\times}^{j} z^{j}\right) \phi_{i}+P_{i}(z)
$$

where $M_{\times}$is the adjacency matrix of $D_{\times}$and $\psi_{i}$ and $\phi_{i}$ are the appropriate start and finish vectors. The term $P_{i}(z)$ is a polynomial which counts the appropriate $m$-compositions with $m<\sigma$. The number of sequences $y_{1}, \ldots, y_{n+1}$ is then

$$
\left[z^{m-\delta}\right] \prod_{i=1}^{n+1} c^{\langle i\rangle}(z)
$$

By Theorem 2.1 and Theorem 2.2,

$$
\prod_{i=1}^{n+1} c^{\langle i\rangle}(z)=A^{\prime} \frac{1}{(1-B z)^{n+1}}+O\left((1-B z)^{-n}\right), \quad z \rightarrow 1,
$$

and

$$
\left[z^{m-\delta}\right] \prod_{i=1}^{n+1} c^{\langle i\rangle}(z)=m^{n} A^{\prime \prime} \cdot B^{m}\left(1+O\left(m^{-1}\right)\right), \quad m \rightarrow \infty .
$$

There is a finite set of possible values for the objects we fixed and from the assumptions we know $n$ attains the value $r-\mu$, so we conclude the result. 
Notation 2.9. For a sequence of random variables $X_{0}, X_{1}, \ldots$, we write $X_{n} \Rightarrow X_{0}$ to denote that the sequence converges in distribution to $X_{0}$.

Proposition 2.11. Let $D$ be a strongly connected, aperiodic digraph with at least 2 vertices. Let $A \subseteq V(D) \times V(D)$ be a nonempty set of allowed start-finish vertex pairs. Let $\Xi \subset V(D)$ be a nonempty set of designated vertices such that there are arbitrarily long walks in $D-\Xi$ and/or $D[\Xi]$, e.g. $D-\Xi$ is strongly connected. Let $X_{m}$ be the number of vertices of $\Xi$ in a uniform random walk of length $m$ in $D$ where the initial and final vertices are found as a pair in $A$. Then $E\left(X_{m}\right) \sim c_{1} m, \operatorname{Var}\left(X_{m}\right) \sim c_{2} m$ where $c_{1}, c_{2}>0$ do not depend on $A$, and

$$
\frac{X_{m}-E\left(X_{m}\right)}{\sqrt{\operatorname{Var}\left(X_{m}\right)}} \Rightarrow N(0,1) .
$$

Proof. Say $V(D)=n$ and fix an ordering $v_{1}, \ldots, v_{n}$ on $V(D)$. We use $u$ as an indeterminate to mark occurrences of $\Xi$. Let $C$ be an $n \times n$ matrix where

$$
[C]_{i, j}=\left[\left(v_{i}, v_{j}\right) \in A\right]\left(u\left[v_{i} \in \Xi\right]+\left[v_{i} \notin \Xi\right]\right)
$$

and let $T$ be an $n \times n$ matrix where

$$
[T]_{i, j}=\left[\left(v_{i}, v_{j}\right) \in E(D)\right]\left(u\left[v_{j} \in \Xi\right]+\left[v_{j} \notin \Xi\right]\right)
$$

The matrix $T$ is known as the transfer matrix. Then $\left[u^{r}\right] \sum_{i, j}[C]_{i, j}\left[T^{m-1}\right]_{i, j}$ is the number of walks of length $m$ in $D$ with $r$ occurrences of $\Xi$ with start and finish vertices allowed by $A$.

Theorem 1 in [BRW83] establishes limiting distributions for secondary parameters in the context of the transfer matrix method. It can be applied to obtain the result if we verify that there is a vertex $v \in V(D)$ and positive integer $k$ such that there are walks 
from $v$ to $v$ of length $s$ with differing numbers of terms in $\Xi$. Assume WLOG that there are arbitrarily long walks in $D-\Xi$ and suppose $v \notin \Xi$. Let $W$ be a sufficiently long walk from $v$ to $v$ in $D-\Xi$. There are walks from $v$ to $v$ which visit $\Xi$, and by aperiodicity of $D$ such a walk exists of the exact length of $W$, so we are finished.

Clearly if $\Xi=\emptyset$ or $\Xi=V(D)$ the number of occurrences of $\Xi$ is trivial. We note that de Bruijn graphs and their derived digraphs are always aperiodic and strongly connected and there are always arbitrarily long walks at the vertices with loops.

We quote a helpful theorem on convergence in distribution.

Theorem 2.4 (Slutsky). Assume $X_{n}$ and $Y_{n}$ are random variables for $n \geq 1$. Also assume that $X_{n}$ converges in distribution to a random variable $X$ and $Y_{n} \Rightarrow c$ where $c \in \mathbb{R}$. Then $X_{n}+Y_{n} \Rightarrow X+c$ and $X_{n} Y_{n} \Rightarrow X \cdot c$.

Proof. See [Gut05, Theorem 11.4].

Notation 2.10. If $f(z, \ldots)$ is a power series, we use $D_{z}$ to denote the derivative $(d / d z) f(z, \ldots)$.

Example 2.5. We look at compositions over $\mathbb{Z}_{2}$ and keep track of occurrences of $U=\{00,11\}$. The relevant de Bruijn graph $D$ has span $\sigma=2$. For the following we fix a particular ordering $v_{i}$ on the vertices of $D_{\times}$. We use the indeterminates $u$ and $z$ to mark length and total. We define start vector

$$
\psi=z^{2}\left[\begin{array}{llllllll}
u & u & 0 & 0 & 0 & 0 & 1 & 1
\end{array}\right]^{\top},
$$

where $\psi(i)$ is 0 for non-start vertices, $z^{\sigma} u$ for start vertices corresponding to $U$, and 
$z^{\sigma}$ otherwise. The finish vector for compositions of 0 is

$$
\phi_{0}=\left[\begin{array}{llllllll}
1 & 1 & 1 & 1 & 0 & 0 & 0 & 0
\end{array}\right]^{\top} .
$$

The matrix $C$ from Proposition 2.11 is then $\psi \phi_{0}^{\top}$. The transfer matrix is

$$
T=\left[\begin{array}{llllllll}
u & 0 & 0 & 0 & 0 & 0 & 1 & 0 \\
0 & 0 & 0 & 1 & 0 & u & 0 & 0 \\
0 & 0 & 0 & 1 & 0 & u & 0 & 0 \\
u & 0 & 0 & 0 & 0 & 0 & 1 & 0 \\
0 & 0 & 1 & 0 & u & 0 & 0 & 0 \\
0 & u & 0 & 0 & 0 & 0 & 0 & 1 \\
0 & u & 0 & 0 & 0 & 0 & 0 & 1 \\
0 & 0 & 1 & 0 & u & 0 & 0 & 0
\end{array}\right],
$$

where $T_{i, j}=0$ if $\left(v_{i}, v_{j}\right) \notin E\left(D_{\times}\right), T_{i, j}=u$ if $\left(v_{i}, v_{j}\right) \in E\left(D_{\times}\right)$and $v_{j}$ corresponds to $U$, and $T_{i, j}=1$ otherwise. We define

$$
P(z, u)=z^{\sigma-1} \psi^{\top}(I-z T)^{-1} \phi_{0},
$$

getting that $\left[z^{m} u^{r}\right] P(z, u)$ is the number of $m$-compositions of 0 over $\mathbb{Z}_{2}$ with $r$ occurrences of $U$.

Let $X_{m}^{\langle 0\rangle}$ be the number of occurrences of $U$ in a uniform-random $m$-composition of 0 . We have

$$
E\left(X_{m}^{\langle 0\rangle}\right)=\frac{\left.\left[z^{m}\right] D_{u} P(z, u)\right|_{u=1}}{\left[z^{m}\right] P(z, 1)}=\frac{1}{2} m+O(1),
$$

and

$$
\begin{aligned}
\operatorname{Var}\left(X_{m}^{\langle 0\rangle}\right) & =\frac{\left.\left[z^{m}\right] D_{u}^{2} P(z, u)\right|_{u=1}}{\left[z^{m}\right] P(z, 1)}+\frac{\left.\left[z^{m}\right] D_{u} P(z, u)\right|_{u=1}}{\left[z^{m}\right] P(z, 1)}-\left(\frac{\left.\left[z^{m}\right] D_{u} P(z, u)\right|_{u=1}}{\left[z^{m}\right] P(z, 1)}\right)^{2} \\
& =\frac{1}{4} m+O(1) .
\end{aligned}
$$


So Proposition 2.11 (and an application of Theorem 2.4) entail

$$
\frac{X_{m}^{\langle 0\rangle}-\frac{1}{2} m}{\frac{1}{2} \sqrt{m}} \Rightarrow N(0,1) .
$$

Asymptotic joint distributions and local limit phenomena are derivable, under conditions, based on [BRW83] and/or [Ber+03]. One can also analyze additional parameters (longest runs, etc.) in analogy to the existing local restriction theory. However in these matters as in Proposition 2.11 one expects to get results identical to those for words (disregarding the total) since arbitrary start and finish requirements do not affect asymptotic distributions.

Lemma 2.7. Let the greatest letter in a subword pattern $\tau$ be $j^{*}$. Assume $k \geq 2$ and $k \geq j^{*}$. If $\tau$ has length $p+1 \geq 2$ and $\tau$ is not $1^{p} 2$ and its symmetries $\left(12^{p}, 2^{p} 1\right.$, and $\left.21^{p}\right)$, there is a strongly connected de Bruijn subgraph $D$ with span $\sigma \geq p$ whose walks represent words over $[k]$ that avoid $\tau$.

Proof. The patterns $1^{p} 2$ do not satisfy this because $1^{p}$ and $2^{p}$ are both allowed but there is no allowed sequence of the form $1^{p} w 2^{p}$ where $w$ is some word.

Let $\tau=(\tau(1), \ldots, \tau(\sigma+1))$. Let $D$ be the de Bruijn subgraph of span $\sigma$ representing $k$-ary words avoiding $\tau$. Let $x=(x(1), \ldots, x(\sigma))$ and $y=(y(1), \ldots, y(\sigma))$ be vertices of $D$. We proceed by cases, establishing either that $x \longrightarrow y$ and $y \longrightarrow x$ or $1^{\sigma} \longrightarrow x$ and $x \longrightarrow 1^{\sigma}$.

Case 1: $j^{*}=1$. If $x(\sigma) \neq y(1)$, then the concatenation $x y$ is allowed. Otherwise, take $c \neq x(\sigma)=y(1)$ and then $x c y$ is allowed.

Case $2: j^{*} \geq 3$. Assume WLOG $\tau(1)>1$. Then $1^{\sigma} x$ is always allowed. If $\tau(\sigma)>1$ 
then $x 1^{\sigma}$ is allowed too. Otherwise $\tau(\sigma)=1$ and $x k^{\sigma} 1^{\sigma}$ is allowed.

Case 3: $j^{*}=2$. Assume WLOG $\tau(1)=2$. Again $1^{\sigma} x$ is allowed. If $\tau(\sigma)=2$ then $x 1^{\sigma}$ is allowed too. If $\tau(\sigma)=1$ and $\tau$ is not monotonic then $x k^{\sigma} 1^{\sigma}$ is allowed. Finally, if $\tau=2^{p} 1^{q}$ with $p, q>1$ then $x(k 1)^{p} 1^{p-1}$ is allowed.

This shows that a satisfactory digraph exists with span $\sigma$. It is now easily seen that a digraph with greater span would also be strongly connected.

Lemma 2.8. Let $G$ be a totally ordered finite group and let $\tau$ be a subword pattern of length at least 2 other than $1^{p} 2$ and its symmetries $\left(12^{p}, 2^{p} 1\right.$, and $\left.21^{p}\right)$. If $j^{*}$ is the greatest letter in $\tau$, assume $|G| \geq \max \left(3, j^{*}\right)$. The de Bruijn subgraph $D$ with span $\sigma=|\tau|$ representing compositions over $G$ avoiding $\tau$ is such that $D_{\times}$is strongly connected and aperiodic.

Proof. Let $a, b \in G$ be distinct and both nonzero.

We show strong connectedness of $D_{\times}$. Let $c=-a$. If $\tau=1^{\sigma}$, then the composition $0^{\sigma-1} a 0 b 0 c 0^{\sigma-1} a$ exhibits a path $\left(0^{\sigma-1} a, 0\right) \longrightarrow\left(0^{\sigma-1} a, b\right)$. Otherwise, if $\tau \neq i^{p} j i^{q}$ where $p, q \geq 1$ and $i \neq j$, then $0^{\sigma} a 0^{\sigma}$ is allowed and therefore $\left(0^{\sigma}, 0\right) \longrightarrow\left(0^{\sigma}, a\right)$ in $D_{\times}$. Finally, if $\tau=i^{p} j i^{q}$, let $n$ be the order of $\sigma a$. Then $0^{\sigma} a^{\sigma n} a 0^{\sigma}$ shows $\left(0^{\sigma}, 0\right) \longrightarrow\left(0^{\sigma}, a\right)$. We show aperiodicity of $D_{\times}$. The vertex $\left(0^{\sigma}, 0\right)$ exists in $D_{\times}$and has a loop if and only if $\tau \neq 1^{\sigma}$. For the pattern $1^{\sigma}$ where $\sigma \geq 3$, the two sequences $b^{\sigma-1} 0 b^{\sigma-1} 0$ and $b^{\sigma-1} 00 b^{\sigma-1} 0$ are allowed and correspond to walks $\left(b^{\sigma-1} 0,0\right) \longrightarrow\left(b^{\sigma-1} 0,(\sigma-1) b\right)$ with lengths differing by 1 . Lastly, if $\tau=11$, the compositions $a b a b$ and $a b 0 a b$ represent paths from $((a, b), 0)$ to $((a, b), a+b)$ with lengths differing by 1 .

Theorem 2.5. Let $G$ be a finite group with a total order and let $\tau$ be a subword 
pattern of length at least 2 not $1^{p} 2$ or its symmetries $\left(12^{p}, 2^{p} 1\right.$, and $\left.21^{p}\right)$. If $j^{*}$ is the greatest letter in $\tau$, assume $|G| \geq \max \left(3, j^{*}\right)$. The number of $m$-compositions of $a \in G$ containing $r$ occurrences of $\tau$ is

$$
A_{r} m^{r} B^{m}\left(1+O\left(m^{-1}\right)\right), \quad A_{r}>0, B>1, m \rightarrow \infty .
$$

If $X_{m}^{\langle a\rangle}$ is the number of occurrences of $\tau$ in a uniform random $m$-composition of $a \in G$ then

$$
\frac{X_{m}^{\langle a\rangle}-E\left(X_{m}^{\langle a\rangle}\right)}{\sqrt{\operatorname{Var}\left(X_{m}^{\langle a\rangle}\right)}} \Rightarrow N(0,1) .
$$

Proof. To satisfy the requirements of Theorem 2.3 we must show that there are arbitrarily long compositions with a single occurrence of $\tau$. Let $a$ be the minimal element of $G$ and suppose $b>a$. If $\tau=1^{p}$, then $\cdots a b a b a^{p} b a b a \cdots$ is such a composition. If $\tau \neq q^{p}$, let $y$ be an occurrence of $\tau$ with minimal-valued parts; then $a \cdots a y a \cdots a$ is such a composition. We can now apply Lemma 2.8, Theorem 2.3, Proposition 2.3, and Proposition 2.11 to conclude the result.

We are immediately able to modify results where they are available for words containing subwords patterns.

Proposition 2.12. Let $G$ be a totally ordered group with $|G|=k$, e.g. $\mathbb{Z}_{k}$ where $0<1<\cdots<k-1$. Define

$$
C(y)=\left[q^{r}\right] \frac{1}{1-y-\sum_{p=3}^{k} \sum_{j=0}^{p-3}\left(\begin{array}{c}
p-3 \\
j
\end{array}\right)\left(\begin{array}{c}
k \\
p+j
\end{array}\right) y^{p+j}(q-1)^{p-2}},
$$

as in [HM10, p. 112]. Let $\rho>0$ be the radius of convergence of $C(y)$, and let $A_{r}=\lim _{y \rightarrow \rho}\left((1-y / \rho)^{r+1} C(y)\right)$.

The number of m-compositions of $a \in G$ containing $r$ occurrences of the subword 


\begin{tabular}{|c|rrr|}
\hline$m$ & 0 & 1 & 2 \\
\hline 2 & 5 & 5 & 5 \\
3 & 23 & 23 & 23 \\
4 & 105 & 105 & 105 \\
5 & 478 & 477 & 477 \\
6 & 2171 & 2171 & 2171 \\
7 & 9869 & 9868 & 9868 \\
8 & 44861 & 44861 & 44861 \\
9 & 203930 & 203930 & 203930 \\
10 & 927032 & 927032 & 927033 \\
11 & 4214147 & 4214147 & 4214147 \\
12 & 19156861 & 19156861 & 19156865 \\
13 & 87084158 & 87084158 & 87084158 \\
14 & 395871195 & 395871195 & 395871198 \\
15 & 1799569607 & 1799569609 & 1799569610 \\
16 & 8180566793 & 8180566793 & 8180566793 \\
\hline
\end{tabular}

Table 3: Counts of $m$-compositions of some $a$ avoiding 132 over $\mathbb{Z}_{5}$.

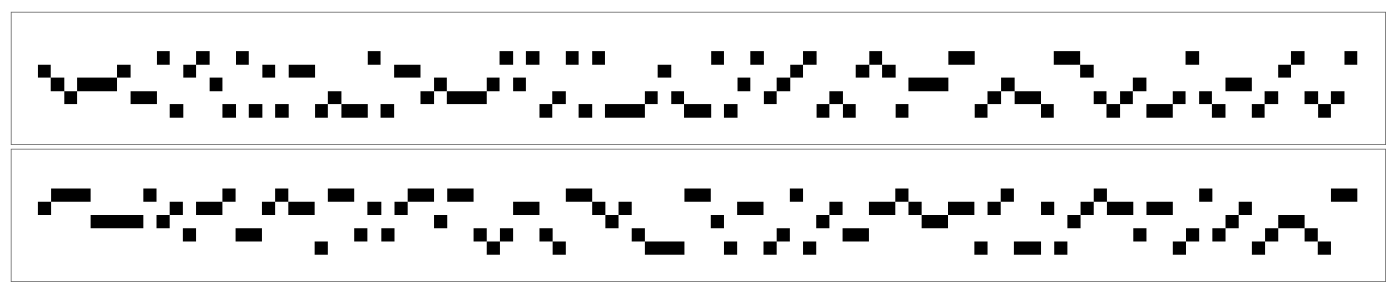

Figure 11: Uniform-randomly generated 100-compositions of 0 (above) and 1 (below) over $\mathbb{Z}_{5}$ which avoid 132 .

pattern 123 is

$$
\frac{1}{k} A_{r} m^{r}\left(\frac{1}{\rho}\right)^{m}\left(1+O\left(m^{-1}\right)\right), \quad m \rightarrow \infty .
$$

Proof. Theorem 4.30 in [HM10] states that $\left[y^{m}\right] C(y)$ is the number of $m$-compositions with any total containing $r$ occurrences of 123 . The result then follows from Theorem 2.5 .

Table 3 shows counts of $m$-compositions of $a$ avoiding $\tau=132$ over $\mathbb{Z}_{5}$. Figure 11 gives randomly selected compositions avoiding 132 over $\mathbb{Z}_{5}$. Figure 12 gives the same for compositions avoiding 121 . 


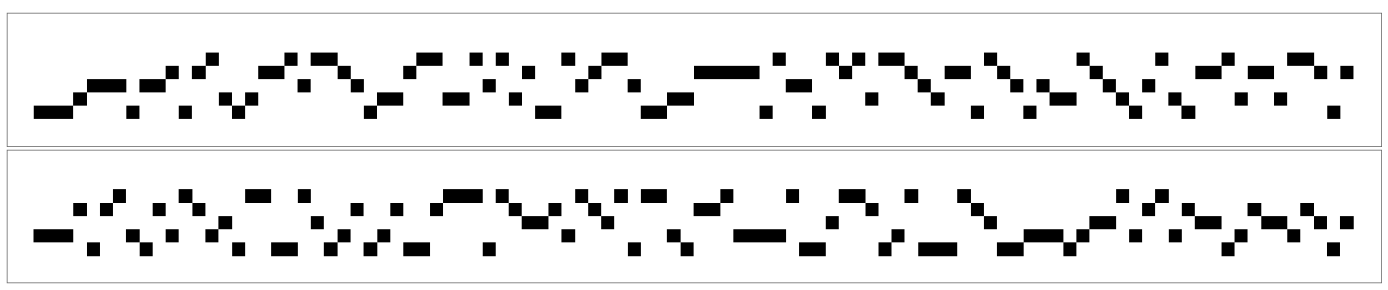

Figure 12: Uniform-randomly generated 100-compositions of 0 (above) and 1 (below) over $\mathbb{Z}_{5}$ which avoid 121 .

\subsection{Note on minimization of transfer matrices}

Given a transfer matrix (adjacency matrix) $T$, we may compute counting sequences by raising $T$ to a power, and if $T$ has a simple enough structure, we may even be able to extract a closed form expression for the counting sequence, or learn other information. A simple transfer matrix corresponds to a simple digraph $D$. Locally restricted compositions over a finite set constitute a regular language, so equivalently we may say we are interested in simple finite automata. In those terms, a question arises: Given a finite automaton $A$, when is it possible to find a smaller automaton $A^{\prime}$ such that $A$ and $A^{\prime}$ are equivalent for counting purposes?

Definition 2.5. As in [RE04], two deterministic finite automata (DFAs) $A, A^{\prime}$ are weakly equivalent if for each integer $m \geq 0$, the automata $A$ and $A^{\prime}$ accept the same number of words of length $m$.

Our question is largely answered by an algorithm given in [RE04, § 4.2], which we refer to as the Ravikumar-Eisman algorithm. The algorithm is given a DFA $A$ and returns a weakly equivalent DFA $A^{\prime}$ with the same number or fewer states. While the Ravikumar-Eisman algorithm is not guaranteed to find the smallest such $A^{\prime}$, it is shown to be practically useful and no better technique is currently available. Roughly speaking, the Ravikumar-Eisman algorithm works by finding states which 


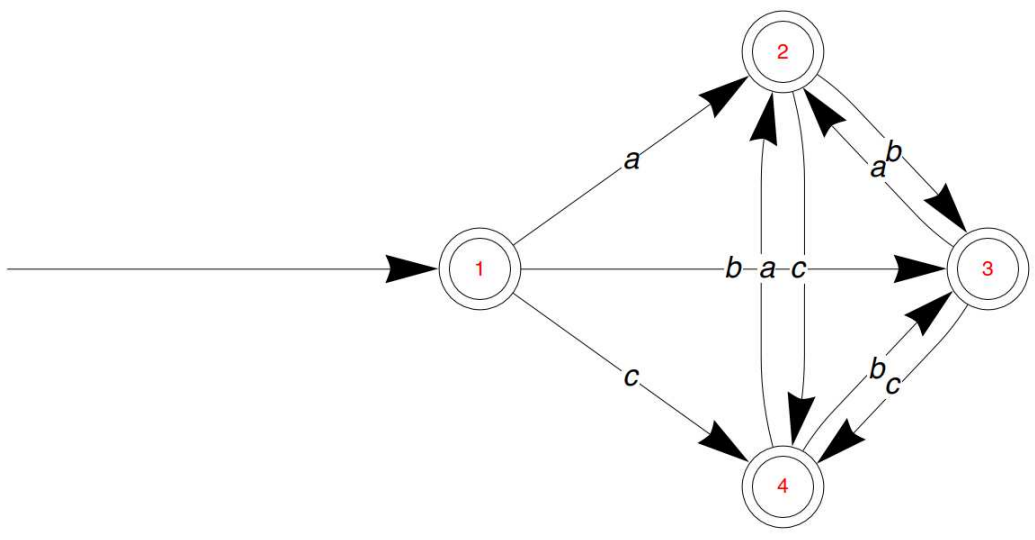

Figure 13: A DFA that accepts 3-ary Carlitz words over $\{a, b, c\}$.

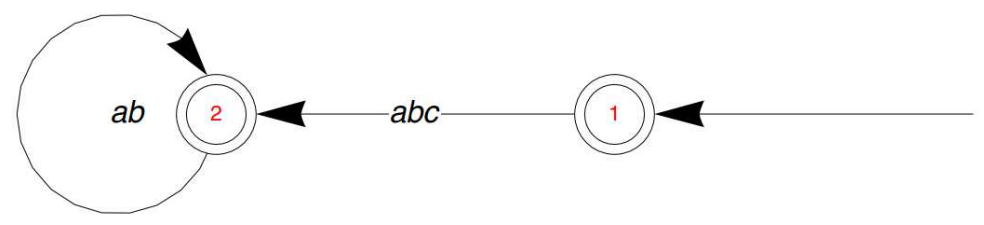

Figure 14: A DFA weakly equivalent to one that accepts 3-ary Carlitz words.

are equivalent in the weak sense (there are equal numbers of accepted words of length $m$ starting at each state for each $m$ ); these states are then merged.

Example 2.6. Figure 13 shows a naive automaton for Carlitz/Smirnov words on the alphabet $\{a, b, c\}$. In fact this automaton is minimal in the usual sense of number of states. However, there is a length-preserving bijection between 3-ary Carlitz words and the language accepted by the automaton in Figure 14, which is returned by the Ravikumar-Eisman algorithm.

For general $k$-ary Carlitz words, we still need only a 2 -state DFA $A^{\prime}$ rather than the naive $k+1$ states. Suppose we number the start state of $A^{\prime}$ as 1 and the other state as 2 . Let $f_{i}(m), i=1,2$ be the number of $m$-words accepted by $A^{\prime}$ if state $i$ were the 
start state. Either by converting to a regular grammar or using the transfer matrix method we get $f_{2}(m)=(k-1)^{m}, f_{1}(m)=k f_{2}(m-1)$ which allows us to conclude (the obvious) $f_{1}(m)=k(k-1)^{m-1}$.

If we generalize $k$-ary Carlitz words to avoidance of the subword pattern $1^{p}$, a naive automaton $A$ with $k^{p}+O(1)$ states has weakly equivalent automaton $A^{\prime}$ with $p+O(1)$ states including, for each $1 \leq i<p$, a state representing words ending with a run of length $i$. Similar phenomena are seen for other subword patterns, with the general theme that simpler patterns have simpler automata.

We can also consider the following refinement of weak equivalence for multivariate counting.

Definition 2.6. Take two DFAs $A$ and $A^{\prime}$ that recognize a language over a k-ary alphabet. Then $A$ and $A^{\prime}$ are completely weakly equivalent if for all $j_{1}, \ldots, j_{k}$, the DFAs $A$ and $A^{\prime}$ accept an equal number of words with $j_{i}$ letters $i$, for $1 \leq i \leq k$.

There is a brute-force algorithm for exact minimization of DFAs according to complete weak equivalence: Given a DFA $A$, enumerate all smaller DFAs $A^{\prime}$ in ascending order by size. Extract the multivariate rational generating functions for $A$ and $A^{\prime}$ where there is an indeterminate symbol marking each letter, and compare by subtracting and testing for 0 .

A straightforward generalization of the faster Ravikumar-Eisman algorithm to the multivariate problem depends on generalizing Lemmas A1 and A2 in [SH85] from sequences of real numbers to sequences of real polynomials. We give this generalization.

Lemma 2.9. Let $\Xi$ be a finite nonempty index set. For all $\xi \in \Xi$ let $A_{\xi}: \mathbb{Z}_{\geq 0} \rightarrow$ 
$\mathbb{R}\left[x_{1}, \ldots, x_{k}\right]$ satisfy

$$
A_{\xi}(n+1)=\sum_{t \in \Xi} c_{\xi, t} A_{t}(n), \quad n \geq 0
$$

where $c_{\xi, t} \in \mathbb{R}\left[x_{1}, \ldots, x_{k}\right]$. Then each $A_{\xi}$ satisfies a linear difference (recurrence) equation of degree $|\Xi|$ or less with coefficients in $\mathbb{R}\left[x_{1}, \ldots, x_{k}\right]$.

Proof. The proof of Lemma A1 in [SH85] requires no modification to prove this result, except that linear algebra is done over $\mathbb{R}\left(x_{1}, \ldots, x_{k}\right)$ rather than $\mathbb{R}$.

Lemma 2.10. Let $A, B: \mathbb{Z}_{\geq 0} \rightarrow \mathbb{R}\left[x_{1}, \ldots, x_{k}\right]$ be sequences satisfying linear difference equations of degrees $a$ and $b$ with coefficients in $\mathbb{R}\left[x_{1}, \ldots, x_{k}\right]$. If for $0 \leq n \leq a+b$ we have $A(n)=B(n)$ then the sequences $A(n)$ and $B(n)$ are identical for all $n$.

Proof. As above for [SH85, Lemma A2].

\subsection{Note on weighted trees}

The number of unweighted binary plane trees avoiding certain local structures is found in [Row10]. In that paper, $\$ 5$ gives an algorithm to compute a system of algebraic equations specifying the relevant generating function. An extension to ternary and $m$-ary trees is in $[\mathrm{Gab}+12, \S 3]$. Enumeration of unweighted trees by number of local occurrences (not just avoidance) is done in [Chy+08]. The paper [Dai+12] considers global pattern avoidance, still in unweighted trees.

Locally restricted trees weighted by a finite group are those that avoid subgraphs from a fixed set $\Xi$ of weighted trees, where the set $\Xi$ has a maximum size. We specifically consider rooted trees where there is a directed edge from parent to child. 
Plane trees correspond, for example, to the family of trees where the vertex set is $[m]$, parents are less than children, and all vertices at a given depth form a contiguous interval of integers. For non-abelian groups, there must be a stipulated ordering on tree vertices such as depth-first search, in order to define the total of a tree. We require that the order be recursive, in the sense that the total of the tree must be a sum made from the weight of the root and the sums of subtrees rooted at children of the root. Variations on trees with similar enumerative properties include functional graphs, directed acyclic graphs, and cactus graphs.

Example 2.7. Let us consider a tree $T$ with weights from $\mathbb{Z}_{2}$ where the first 2 levels are of the form

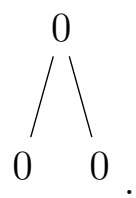

If $T$ avoids parent-child-grandchild paths with the same weight on all 3 vertices, sibling subtrees are independent but no level-3 vertex of $T$ may have the weight 0 .

An alternative visual representation of weighted trees for groups with some total order uses a color behind vertices where darker means greater. This plotting technique works well for larger trees because of higher visibility, although it is less precise. An example is shown in Figure 15.

It is straightforward (albeit a little cumbersome) to show that the generating function $T_{a}(z)$, counting locally restricted trees with total $a \in G$, where $z$ marks number of vertices, is expressible in terms of a system of algebraic equations. This is, of course, common in tree enumeration as in examples in [Row10], [FS09, §I.5], [Drm09], et cetera. The theory of coefficients of algebraic functions in, e.g. [FS09, §VII.6.1] may be 

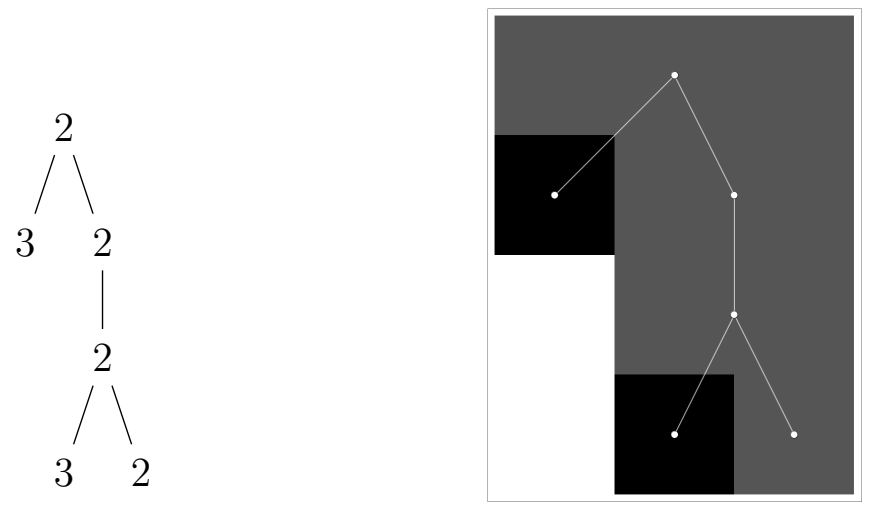

Figure 15: A tree weighted by $\mathbb{Z}_{4}$ shown in two plotting styles.

applied, under conditions, to derive the usual $\left[z^{m}\right] T_{a}(z) \sim A_{a} m^{-1 / 2} B^{m}, A_{a}>0, B>1$. The works [Chy+08; Drm09] conduct analysis of the number of pattern occurrences in uniform random unweighted trees and show convergence in distribution to the standard normal after normalization; we expect that their method applies similarly in the present context.

Finally, we note that computing expansions of multivariate algebraic series is possible using software packages available for computer algebra systems. Newton iteration is a relatively efficient option. The package Genfunlib [Mac14] for Mathematica implements Newton iteration as the command CoefsByNewton, but only for single equations, so there is a preliminary step of eliminating all but one component of the original system. An example expansion follows of the solution to $f(z, u)=u+$ $z\left(f(z, u)^{2}+f(z, u)\right)$.

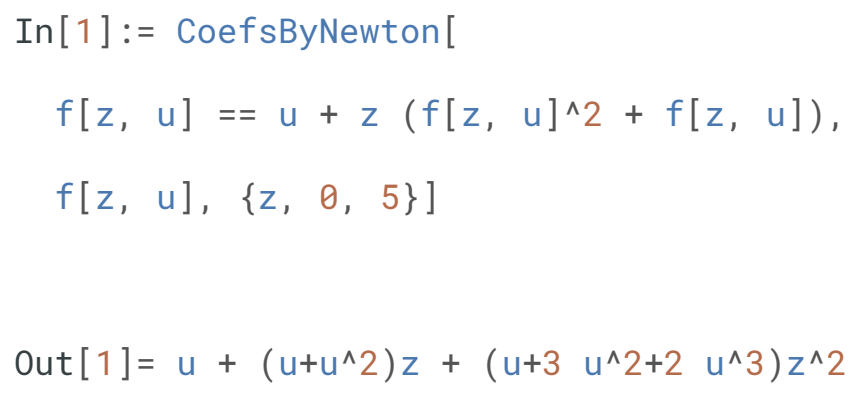


$+\left(u+6 u^{\wedge} 2+10 u^{\wedge} 3+5 u^{\wedge} 4\right) z^{\wedge} 3+\left(u+10 u^{\wedge} 2+30 u^{\wedge} 3+35 u^{\wedge} 4+14 u^{\wedge} 5\right) z^{\wedge} 4$

$+\left(u+15 u^{\wedge} 2+70 u^{\wedge} 3+140 u^{\wedge} 4+126 u^{\wedge} 5+42 u^{\wedge} 6\right) z^{\wedge} 5+0[z]^{\wedge} 6$ 


\section{Locally cyclically restricted compositions}

Remark 3.1. In the language of $\S 1$, cyclically restricted compositions are weighted directed cycles where we track the total weight. Weighted undirected cycles may be counted in a similar manner. Sets of cycles, i.e. 2-regular graphs, are also closely related.

\subsection{Compositions over a finite group}

In $\S 2.1$ we represented compositions by walks on any de Bruijn subgraph $D$ over $\mathrm{SEQ}_{\sigma}(G)$. Within the current section and $\S 4.1$ we slightly specialize the possibilities for $D$ as follows. If $\sigma \geq 1$ is the span, let $\bar{D}$ be the $\sigma$-dimensional de Bruijn graph on $G$, let $U \subset V(\bar{D})$, and let $D=\bar{D}-U$. Note that given such a digraph $D$, the set $U$ is uniquely determined.

An $m$-composition $x$ is locally cyclically restricted according to $D$ if

$$
(x(1), \ldots, x(m), x(1), \ldots, x(\sigma-1))
$$

avoids $U$ as subwords. A number of observations about this definition should be made. First, $m$-compositions where $m<\sigma$ do not correspond to walks over $D$ but may or may not be cyclically restricted according to $D$. Second, for $m<\sigma$, we are technically departing from the isomorphic nature of pattern occurrences in the language of $\S 1$, and really this corresponds to homomorphic pattern occurrences. We do not remark on this point further.

Let $\mathcal{C}_{a}(m ; D)$ be the set of all $m$-compositions of $a$ that are cyclically restricted ac- 
cording to $D$, and define

$$
c_{a}(m ; D)=\left|\mathcal{C}_{a}(m ; D)\right|, \quad C_{a}(z ; D)=\sum_{m \geq 0} c_{a}(m ; D) z^{m}
$$

Lemma 3.1. For $v \in V(D)$, let $\Sigma^{\prime} v=v(1)+\cdots+v(\sigma-1)$. For $m \geq \sigma$ we have

$$
c_{a}(m ; D)=\sum_{\substack{v \in V(D) \\ u \in N^{-}(v)}} p_{a+\Sigma^{\prime} v}(m+\sigma-1 ; D,\{v\},\{u\})
$$

for $a \in G$.

Proof. If $m \geq \sigma$, consider a walk $w_{1}, \ldots, w_{m}$ in $D_{\times}$, where $w_{1}=\left(v, \sum v\right)$ and $\left(w_{m},\left(v, a+\sum v\right)\right) \in E\left(D_{\times}\right)$. Let $(x(1), \ldots, x(m+\sigma-1))$ be the composition represented by the walk. Then $(x(1), \ldots, x(m))$ is precisely an $m$-composition of $a$ which is cyclically restricted according to $D$.

Proposition 3.1. Fix an ordering on $V\left(D_{\times}\right)$and let $M_{\times}$be the adjacency matrix of $D_{\times}$. For $(v, a) \in V\left(D_{\times}\right)$, let $\xi_{v, a} \in \mathbb{R}^{\left|V\left(D_{\times}\right)\right|}$be the indicator vector for vertex $(v, a)$. Then for $m \geq \sigma$,

$$
c_{a}(m ; D)=\sum_{\substack{v \in V(D) \\ u \in N^{-}(v)}}\left(\xi_{v, \Sigma v}\right)^{\top} M_{\times}^{m-1} \xi_{u, a+\Sigma^{\prime} v}
$$

Proof. This follows from Lemma 3.1 and Proposition 2.1.

Proposition 3.2. Assume $D$ is regular. We have either $c_{a}(m ; D)=0$ or

$$
c_{a}(m ; D)=A_{a} \cdot B^{m}\left(1+O\left(\theta^{m}\right)\right), \quad m \rightarrow \infty
$$

for $A_{a}, B>0,0 \leq \theta<1$.

Proof. This follows from Lemma 3.1 and Proposition 2.4. 
Proposition 3.3. Assume $D_{\times}$is strongly connected and aperiodic. Then $c_{a}(m ; D)=$ $A \cdot B^{m}\left(1+O\left(\theta^{m}\right)\right)$ where $A$ does not depend on $a \in G$.

Proof. This follows from Lemma 3.1 and Theorem 2.1.

Let $x=(x(1), \ldots, x(m))$ and $y=(y(1), \ldots, y(\sigma))$ be compositions. A local cyclic occurrence of $y$ in $x$ is an occurrence of $y$ as a subword in $(x(1), \ldots, x(m), x(1), \ldots, x(\sigma-$ $1))$.

Theorem 3.1. Assume $U$ is nonempty and suppose $D=\bar{D}-U$ is regular with strongly connected derived digraph $D_{\times}$.

For $u \in V(\bar{D})$, let $\mu(u)$ be the minimum number of occurrences of $U$ in a composition in $\mathcal{P}\left(\bar{D},\{u\}, N^{-}(u)\right)$ with at least 1 occurrence of $V(D)$. Let $\mu$ be the minimal such $\mu(u)$. Assume for all sufficiently large values of $m$ there exist compositions in $\mathcal{P}(m ; \bar{D}, V(D), V(D))$ with exactly 1 occurrence of $U$, and that $p(m ; D, V(D), V(D)) \sim A \cdot B^{m}$.

If $r \geq \max (\mu, 1), \mu \geq 0$ then the number of $m$-compositions of $a \in G$ with exactly $r$ cyclic occurrences of $U$ is

$$
c_{a}(m, r ; D)=m^{r-\mu} A_{r, \mu} \cdot B^{m}\left(1+O\left(m^{-1}\right)\right), \quad m \rightarrow \infty
$$

Proof. The result follows from Lemma 3.1 and Theorem 2.3.

Theorem 3.2. Assume that $|G| \geq 2$ and that $U \subset \operatorname{SEQ}_{\sigma}(G)$ is non-empty. The number of cyclic occurrences of $U$ in a uniform random $m$-composition of a $\in G$ is asymptotically normal with mean and variance asymptotic to those of the number of occurrences of $U$ in a uniform random word over $G$. 
Proof. This follows directly from Proposition 2.11. If $D_{\times}$is the derived digraph of the de Bruijn graph on $\operatorname{SEQ}_{\sigma}(G)$, the allowed start-finish pairs are all $(u, v) \in V\left(D_{\times}\right)^{2}$ such that $(v, u) \in E\left(D_{\times}\right)$.

We consider some examples of cyclic restrictions.

Proposition 3.4. A composition $x=(x(1), \ldots, x(m))$ is a cyclic Carlitz composition if $(x(1), \ldots, x(m), x(1))$ is a Carlitz composition. The number of cyclic Carlitz mcompositions of $a \in G$ over a finite group $G$ is

$$
\frac{(|G|-1)^{m}}{|G|}\left(1+O\left(\theta^{m}\right)\right), \quad m \rightarrow \infty, 0 \leq \theta<1
$$

provided $|G| \geq 3$.

Proof. First let us consider cyclic Carlitz $m$-words over $[k]$. Assume the first letter is $k$. A cyclic Carlitz (or Smirnov) word is then a sequence of pairs of a single letter $k$ followed by a non-empty Carlitz word on $[k-1]$. Let $\bar{H}_{k}(z)=k z /(1-(k-1) z)$ be the ordinary generating function for non-empty Carlitz words on $[k]$. Thus if $F_{k}(z)$ is the ordinary generating function for cyclic Carlitz words on $[k]$, we have

$$
\begin{aligned}
F_{k}(z) & =k \frac{z \bar{H}_{k-1}(z)}{1-z \bar{H}_{k-1}(z)} \\
& =k \frac{(k-1) z^{2}}{(z+1)(1-(k-1) z)}
\end{aligned}
$$

and

$$
\left[z^{m}\right] F_{k}(z)=(k-1)^{m}+k(-1)^{m}+(-1)^{m+1}, \quad m>1
$$

The above derivation is a special case of Theorem 4 in [Had17]. It remains to recall from Proposition 2.7 that there is a digraph $D$ representing Carlitz compositions such that $D_{\times}$is aperiodic and strongly connected; Proposition 3.3 applies. 
Let $\Xi=\left\{\xi_{1}, \xi_{2}, \ldots\right\}$ be an ordered set. A sequence $w=(w(1), \ldots, w(m))$ over $\Xi$ is $p$-smooth if for all $i=1, \ldots, m-1$ if we have $w(i)=\xi_{j}, w(i+1)=\xi_{k}$ then $|k-j| \leq p$. Additionally, $w$ is $p$-smooth cyclic if $(w(1), \ldots w(m), w(1))$ is $p$-smooth. Research Direction 6.5 in [HM10, p. 239] asks for an explicit formula for the number of $p$-smooth cyclic $k$-ary words of length $m$.

We apply Proposition 3.3 in the case $p=1$.

Proposition 3.5. Let $\mathbb{Z}_{k}$ have ordering $0,1, \ldots, k-1$. Let

$$
C(z)=1+\frac{k z(1+3 z)}{(1+z)(1-3 z)}-\frac{2(k+1) z}{(1+z)(1-3 z)} \frac{U_{k-1}\left(\frac{1-z}{2 z}\right)}{U_{k}\left(\frac{1-z}{2 z}\right)}
$$

be the ordinary generating function for k-ary 1-smooth cyclic words as in [HM10, Exercise 6.10] and [Kno+10; KMM11] where $U_{k}$ is the $k^{\text {th }}$ Chebyshev polynomial of the second kind.

Let $\rho>0$ be the radius of convergence of $C(z)$, and let $A=\lim _{z \rightarrow \rho}(1-z / \rho) C(z)$. Then the number of 1-smooth cyclic $m$-compositions of $i \in \mathbb{Z}_{k}$ is asymptotic to

$$
\frac{1}{k} A \cdot\left(\frac{1}{\rho}\right)^{m}\left(1+O\left(\theta^{m}\right)\right), \quad m \rightarrow \infty, 0 \leq \theta<1 .
$$

Proof. Let $\bar{D}$ be the de Bruijn graph on $\mathbb{Z}_{k}^{2}$, let $U \subseteq \mathbb{Z}_{k}^{2}$ be all $(a, b)$ which are not smooth, and let $D=\bar{D}-U$. In the derived graph $D_{\times}$, for any $i \in \mathbb{Z}$, we exhibit a walk from $((0,0), i)$ to $((0,0), 0)$ by taking the following sequence of elements of $\mathbb{Z}_{k}$. First, take $0,1,2, \ldots,-i-1,-i,-i-1, \ldots, 2,1,0,0$. Let $c=1+2+\cdots+-i-1$. The sum of elements on this sequence is $2 c-i$. Let $n$ be the order of $2 c$ in $\mathbb{Z}_{k}$. Repeat the following $n-1$ times: $0,1,2, \ldots,-i-2,-i-1,-i-1,-i-2, \ldots, 0,0$. The grand total of these sequences concatenated is $2 c-i+(n-1) c=-i$ and thus there is a walk in $D_{\times}$starting at $((0,0), i)$ and ending at $((0,0), 0)$. The digraph $D_{\times}$is 


\begin{tabular}{|c|rr|}
\hline$m$ & 0 & 1 \\
\hline 3 & 19 & 19 \\
4 & 85 & 85 \\
5 & 390 & 385 \\
6 & 1763 & 1763 \\
7 & 8023 & 8016 \\
8 & 36469 & 36469 \\
9 & 165790 & 165790 \\
10 & 753660 & 753660 \\
11 & 3426039 & 3426039 \\
12 & 15574231 & 15574231 \\
13 & 70798118 & 70798118 \\
14 & 321837325 & 321837325 \\
15 & 1463023035 & 1463023045 \\
16 & 6650677797 & 6650677797 \\
\hline
\end{tabular}

Table 4: Counts of $m$-compositions of a cyclically avoiding 132 over $\mathbb{Z}_{5}$.

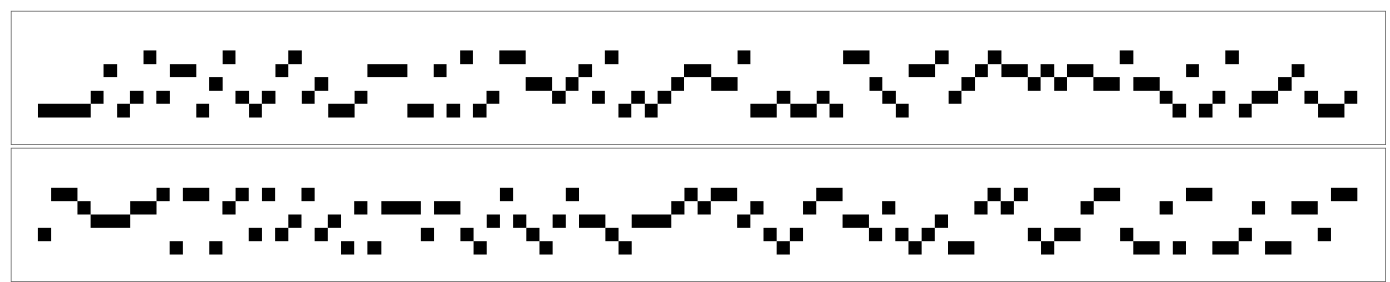

Figure 16: Uniform-randomly generated 100-compositions of 0 (above) and 1 (below) over $\mathbb{Z}_{5}$ which cyclically avoid 132 .

clearly aperiodic since there is a loop at the vertex $((0,0), 0)$. Thus we may apply Proposition 3.3.

Table 4 shows counts of $m$-compositions of $a$ cyclically avoiding $\tau=132$ over $\mathbb{Z}_{5}$. Figure 16 contains uniform randomly generated compositions over $\mathbb{Z}_{5}$ that cyclically avoid 132 .

Remark 3.2. Wheel graphs are a variation on cycles with similar enumerative properties. A wheel graph consists of a cycle $C$ with a vertex $v$ added and (directed) edges from $v$ to each vertex in $C$. 


\subsection{Note on integer compositions}

Let $x=(x(1), \ldots, x(m))$ be an integer composition, i.e. $x(i) \in \mathbb{Z}_{>0}, 1 \leq i \leq m$. To define locally restricted integer compositions with span $\sigma \in \mathbb{Z}_{>0}$ we use a local restriction function $R: \mathbb{Z}_{>0}^{\sigma} \rightarrow\{0,1\}$ which encodes the $\sigma$-tuples that are allowed as a subword inside an integer composition. If $\operatorname{SEQ}\left(\mathbb{Z}_{>0}\right)$ is the set of all integer compositions of any length, define $\bar{R}: \mathrm{SEQ}\left(\mathbb{Z}_{>0}\right) \rightarrow\{0,1\}$ so that $\bar{R}(x)=1$ if and only if $R(x(i), x(i+1), \ldots, x(i+\sigma-1))=1$ for all $1 \leq i \leq m-\sigma+1$, in which case $x$ is allowed according to $R$. As an expedient it is also helpful to define an infinite digraph $\mathcal{D}$ with vertex set $V(\mathcal{D})=R^{-1}(1)$ and where $(u, v) \in E(\mathcal{D})$ if and only if $\bar{R}(u v)=1$. Note that a walk in $\mathcal{D}$ represents a composition obtained by concatenating the vertices; as such, walks do not represent all restricted compositions, only those whose length is a multiple of $\sigma$. In this way the infinite digraph $\mathcal{D}$ is interpreted differently from the de Bruijn graphs used in other sections. We assume there is some

vertex ordering $V(\mathcal{D})=\left\{v_{1}, v_{2}, \ldots\right\}$. We define the transfer operator $T(z)$ formally as the infinite matrix where $[T(z)]_{i, j}=\left[\left(v_{i}, v_{j}\right) \in E(\mathcal{D})\right] z^{\Sigma v_{i}+\Sigma v_{j}}$.

A research direction suggested in $[\operatorname{Had} 17, \S 4]$ is developing a framework for locally cyclically restricted integer compositions. The framework for locally restricted integer compositions in [BC09] can be used with little modification. In this section we follow the definitions of [BC09] with some simplifications.

We say that $x$ is a composition which is cyclically restricted by $R$ if

$$
\bar{R}(x(1), \ldots, x(m), x(1), \ldots, x(\sigma))=1 .
$$


The endpoint operator $E(z, y)$ is a formal infinite matrix defined by

$$
[E(z, y)]_{i, j}=\sum_{k \geq 1} y^{2 \sigma}[T(z)]_{k, i} \sum_{x} z^{\Sigma v_{j}+2 \Sigma x+\Sigma v_{k}} y^{2 \sigma+2|x|},
$$

where the second sum ranges over compositions $x$ with length in $\{0, \ldots, \sigma-1\}$ such that $\bar{R}\left(v_{j} x v_{k}\right)=1$. The endpoint operator plays the role of the start and finish vectors of $[\mathrm{BC} 09]$.

Proposition 3.6. Let $S(z, y)=\sum_{j \geq 0}\left(y^{2 \sigma}\right)^{j} T(z)^{j}$. Define

$$
C\left(z^{2}, y^{2}\right)=\sum_{i, j \geq 1}[S(z, y)]_{i, j}[E(z, y)]_{i, j} .
$$

Then for $m \geq 3 \sigma$, the coefficient $\left[z^{n} y^{m}\right] C(z, y)$ is the number of integer m-compositions of $n$ that are cyclically restricted according to $R$.

Proof. We have

$$
\begin{aligned}
& \sum_{i, j \geq 1}[S(z, y)]_{i, j}[E(z, y)]_{i, j} \\
& =\sum_{k, i, j \geq 1} y^{\sigma} z^{\Sigma v_{k}} y^{2 \sigma}[T(z)]_{k, i}[S(z, y)]_{i, j} y^{\sigma} z^{\Sigma v_{j}} \sum_{x} z^{2 \Sigma x} y^{2|x|}
\end{aligned}
$$

Consider a term

$$
y^{\sigma} z^{\Sigma v_{k}} y^{2 \sigma}[T(z)]_{k, i}[S(z, y)]_{i, j} y^{\sigma} z^{\Sigma v_{j}} \sum_{x} z^{2 \Sigma x} y^{2|x|} .
$$

This is the generating function for restricted compositions of the form

$$
v_{k} v_{i} w v_{j} x v_{k}
$$

where $w$ is a concatenation of vertices in $V(\mathcal{D})$, with $x^{2}$ marking total sum and $y^{2}$ marking length, and such that the final $v_{k}$ does not count. Summing over all $i, j, k$ enumerates cyclically restricted $m$-compositions where $m \geq 3 \sigma$. 
We consider some examples.

Research Direction 4.4 in [HM10] begins as follows. "We say that a sequence (composition, word, partition) $s_{1} \cdots s_{m}$ cyclically avoids a subword $\tau=\tau_{1} \cdots \tau_{\ell}$ if $s_{1} \cdots s_{m} s_{1} \cdots s_{\ell-1}$ avoids $\tau$. For example, the composition 33412 avoids the subword 123, but does not cyclically avoid 123 (since 3341233 contains 123)." The problem is to find the generating function for the number of compositions of $n$ that cyclically avoid a subword pattern of length $k$. We consider the patterns 122 and 321 .

Example 3.1. Compositions cyclically avoiding 122 over $[k]$ take the following form. Either there is no part $k$, the composition only contains $k$, or there is at least one $k$ and and least one other part. In this third case, the subwords between any parts $k$ are nonempty 122-avoiding integer compositions over $[k-1]$ and so is the composition obtained by concatenating the subword after the final $k$ and the subword before the first $k$.

Let $C_{k}(z, u)$ be the generating function for nonempty cyclic 122-avoiding compositions where $z$ marks total and $u$ marks length, and let $P_{k}(z, u)$ be the generating function for nonempty 122-avoiding compositions. The above reasoning yields

$$
C_{k}(z, u)=C_{k-1}(z, u)+\frac{u z^{k}}{1-u z^{k}}+u z^{k} \frac{1}{1-P_{k-1}(z, u) u z^{k}}\left(u D_{u}+1\right) P_{k-1}(z, u),
$$

for $k \geq 2$.

The generating function $P_{k}(z, u)$ is given in [HM10, Theorem 4.35] as

$$
P_{k}(z, u)=\left(1-\sum_{j=1}^{k} z^{j} u \prod_{i=j+1}^{k}\left(1-z^{2 i} u^{2}\right)\right)^{-1}-1
$$

Let $C(z)=\lim _{k \rightarrow \infty} C_{k}(z, 1)$. The coefficients $\left[z^{n}\right] C(z)$ for $n=1, \ldots, 10$ are 
$1,2,4,8,13,28,52,101,196,383$.

Example 3.2. For $\tau=321$, we consider two counting sequences. Let ${ }^{\wedge} 21$ be the pattern 21 except that it only counts if it appears at the beginning of a composition. We count compositions over $[k]$ that avoid both 321 and ${ }^{\wedge} 21$. Such a composition either has no parts $k$ or has at least one $k$. In the latter case, say the composition can be written $\sigma_{1} k \sigma^{\prime}$, where $\sigma_{1}$ is a composition on $[k-1]$ and avoids $\{321, \wedge 21\}$, and $\sigma^{\prime}$ is a composition on $[k]$ avoiding $\{321, \wedge 21\}$. If the composition $\sigma_{1}$ is empty then either $\sigma^{\prime}$ is empty or $k \sigma^{\prime}=k k \sigma^{\prime \prime}$ where $\sigma^{\prime \prime}$ is a composition on $[k]$ avoiding $\{321, \wedge 21\}$. This method proceeds similarly to the proof of Lemma 4.29 in [HM10]. Let $\bar{P}_{k}(z, u)$ be the generating function for compositions avoiding $\{321, \wedge 21\}$ where $z$ marks total and $u$ marks length. This gives

$$
\bar{P}_{k}(z, u)=\bar{P}_{k-1}(z, u)+\left(\bar{P}_{k-1}(z, u)-1\right) u z^{k} \bar{P}_{k}(z, u)+u z^{k}+u^{2} z^{2 k} \bar{P}_{k}(z, u),
$$

for $k \geq 2$. Now we go back to compositions cyclically avoiding just 321 . Case 1: The composition has no part $k$. Case 2: There are at least 2 parts $k$. Such a composition can be written $\sigma_{1} k \sigma^{\prime} k \sigma_{2}$, where $\sigma^{\prime}$ avoids $\left\{321,{ }^{\wedge} 21\right\}$ and $\sigma_{2} \sigma_{1}$ is a composition over $[k-1]$ avoiding $\{321, \wedge 21\}$ Case 3 : There is 1 part $k$. Then the composition is $\sigma_{1} k \sigma_{2}$ where $\sigma_{2} \sigma_{1}$ is a composition over $[k-1]$ avoiding $\left\{321,{ }^{\wedge} 21\right\}$. If $C_{k}(z, u)$ is the generating function for compositions cyclically avoiding 321, we have

$$
\begin{gathered}
C_{k}(z, u)=C_{k-1}(z, u)+u z^{k} \bar{P}_{k}(z, u) u z^{k}\left(u D_{u}+1\right) \bar{P}_{k-1}(z, u) \\
+u z^{k}\left(u D_{u}+1\right) \bar{P}_{k-1}(z, u)
\end{gathered}
$$

for $k \geq 2$.

The paper [BC09] obtains asymptotics for locally restricted integer compositions us- 
ing advanced tools from functional analysis that generalize finite dimensional matrix theory. We expect that analogous results hold for locally cyclically restricted integer compositions.

The method of random generation given in Remark 2.2 achieves an exact uniform distribution but for compositions over an infinite set such as $\mathbb{Z}_{>0}$ its performance becomes poor. Instead we employ a Markov chain Monte Carlo (MCMC) method inspired by the article [ML10] which concerns pattern-avoiding permutations.

The method is as follows. Let $\tau$ be a permutation pattern, i.e. where no letters are repeated, and assume the length of $\tau$ is at least 3 . Let $n, m>0$ be fixed, where $n$ represents a total and $m$ represents a length. (The length $m$ can itself be randomly chosen first using exact counting.) Assume $X_{0}$ is an $m$-composition of $n$ with at most 2 distinct part sizes. Given $X_{h}, h \geq 0$, we generate $X_{h+1}$ as follows. Let $j, k$ be independently selected uniformly at random from $[m]$. If $X_{h}(j)=1$ or $j=k$, then $X_{h+1}=X_{h}$. Otherwise, let $Y$ be the following composition. We have $Y(j)=X_{h}(j)-1$, $Y(k)=X_{h}(k)+1$, and $Y(i)=X_{h}(i)$ for $i \neq j, k$. If $Y$ avoids $\tau$, then $X_{h+1}=Y$, otherwise $X_{h+1}=X_{h}$. A composition avoiding 123 generated by this procedure is shown in Figure 17.

Proposition 3.7. The limiting distribution of the Markov chain $X_{h}$ is uniform over m-compositions of $n$ that avoid $\tau$.

Proof. By the theory of Markov chains [Bil08, Ex. 8.20], it suffices to show that $X_{h}$ is aperiodic, irreducible, and has symmetric transition probabilities. Let $p(x, y)$ be the transition probability from a composition $x$ to $y$. Aperiodicity is clear since $p(x, x)>0$. For symmetry, if $x \neq y$ are compositions with $p(x, y)>0$, then $p(x, y)=$ 


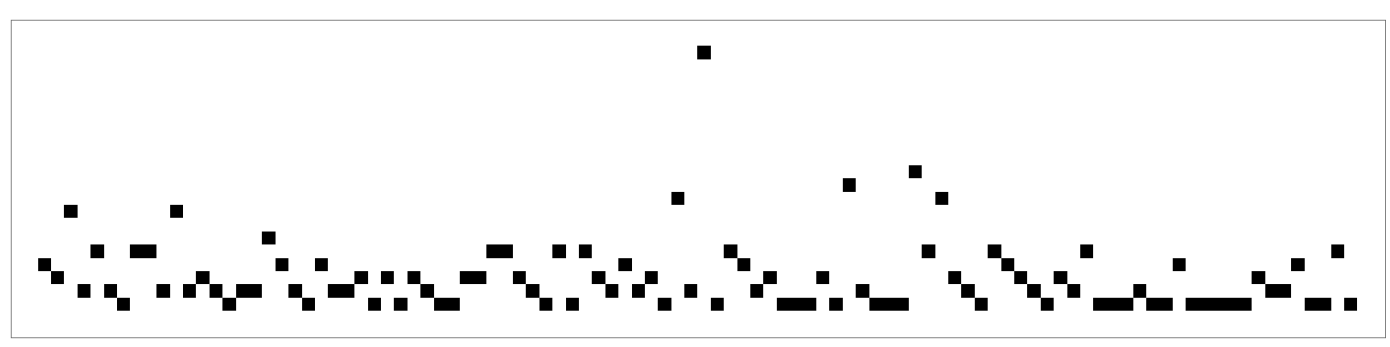

Figure 17: Integer composition of 300 avoiding the subword pattern 123 generated by 10,000,000 iterations of an MCMC method.

$p(y, x)=1 / m^{2}$. With symmetry established, irreducibility requires that for any $x$ there is a sequence of transitions with nonzero probability that lead from $x$ to, say, $X_{0}$. We construct such a sequence. Repeat the following until there are at most 2 distinct part sizes, at which point reaching $X(0)$ is clearly possible. Let $y$ be the current composition and let $K=|\tau|$. Let $j$ be the index of the maximum part in $y$; if this is not unique, take the least such index if $(K, K-1)$ is a subsequence of $\tau$ and take the greatest such index if $(K-1, K)$ is a subsequence of $\tau$. Let $k$ be the index of the minimum part in $y$; if this is not unique, take the least such index if $(1,2)$ is a subsequence of $\tau$, and take the greatest such index if $(2,1)$ is a subsequence of $\tau$. Decrement $y(j)$ and increment $y(k)$. 


\section{Locally restricted compositions with symme- try}

Here we consider locally restricted compositions with symmetry, which corresponds to local patterns in unlabeled weighted digraphs, in the language of $\S 1$. In this section, groups $G$ are assumed to be abelian, since the order of the parts in a composition is no longer well defined.

Although we do not directly invoke it here, general counting with symmetry typically involves Burnside's lemma.

Lemma 4.1 (Burnside). The number of orbits of a permutation group $S$ on a set $X$ is

$$
|X / S|=\frac{1}{|S|} \sum_{s \in S} \operatorname{fix}(s),
$$

where fix $(s)$ is the number of fixed points of $s$.

Further background may be found in [Mac16, §6].

\subsection{Circular compositions}

As in $\S 3.1$, here $G$ is a finite group and $\bar{D}$ is a $\sigma$-dimensional de Bruijn graph over $G$. We speak of digraphs $D=\bar{D}-U$ for some $U \subset V(\bar{D})$.

Lemma 4.2. Assume $x$ is a composition and $x=u \cdots u=u^{d}$. If $u$ is a subword containing $r$ cyclic occurrences of $U$, then $x$ contains $d r$ cyclic occurrences of $U$. 
Proof. A cyclic occurrence of $U$ in a composition is fully determined by the starting index. All cyclic occurrences of $U$ in $x$ must correspond to an occurrence in some $u$, and vice versa.

The circular shift of the finite sequence $(x(1), \ldots, x(m))$ is

$$
(x(j), x(j+1), \ldots, x(m), x(1), x(2), \ldots, x(j))
$$

for some $1 \leq j \leq m$. A circular composition is an equivalence class of cyclically restricted compositions where the equivalence is under circular shift. For example, there are two possible circular Carlitz 3 -compositions over $\mathbb{Z}_{3}$, each with the same total:

$$
\{012,201,120\},\{021,210,102\} \text {. }
$$

Let $\tilde{\mathcal{C}}_{a}(m ; D)$ be the set of all circular $m$-compositions of $a$ that are cyclically restricted according to $D$, and define

$$
\tilde{c}_{a}(m ; D)=\left|\tilde{\mathcal{C}}_{a}(m ; D)\right|, \quad \tilde{C}_{a}(z ; D)=\sum_{m \geq 0} \tilde{c}_{a}(m ; D) z^{m}
$$

Let $P=\mathbb{Z}_{>0} \times G$ be the poset where $(j, a) \preceq(k, b)$ if and only if $j \mid k$ and $(k / j) a=b$. The Moebius function $\mu_{P}$ of $P$ is defined recursively by $\mu_{P}(s, s)=1$ for $s \in P$ and $\mu_{P}(s, u)=-\sum_{s \preceq t \prec u} \mu_{P}(s, t)$ for $s \prec u$ in $P$. A finite sequence is aperiodic if it is not equal to any of its circular shifts.

Proposition 4.1. We have

$$
\tilde{c}_{a}(m ; D)=\sum_{(d, b) \preceq(m, a)} \frac{1}{d} \sum_{\left(d^{\prime}, b^{\prime}\right) \preceq(d, b)} c_{b^{\prime}}\left(d^{\prime} ; D\right) \mu_{P}\left(\left(d^{\prime}, b^{\prime}\right),(d, b)\right) .
$$

Proof. Let acyc $(m, a)$ be the number of aperiodic cyclically restricted $m$-compositions 
of $a$. For any $m$-composition $x$ of $a \in G$, we have $x=u \cdots u=u^{m / d}$ for some aperiodic $u$ and some $d$ which divides $m$, by [Sha08, Theorem 2.3.4]. Thus by Lemma 4.2,

$$
c_{a}(m ; D)=\sum_{(d, b) \preceq(m, a)} \operatorname{acyc}(d, b) .
$$

By the Moebius inversion formula [Sta12, Proposition 3.7.1],

$$
\operatorname{acyc}(m, a)=\sum_{(d, b) \preceq(m, a)} c_{b}(d ; D) \mu_{P}((d, b),(m, a)) .
$$

Now, a circular composition consists of all possible shifts of some composition $x=$ $u^{m / d}$ where $u$ is aperiodic, by [Sha08, Theorem 2.4.2], so

$$
\tilde{c}_{a}(m ; D)=\sum_{(d, b) \preceq(m, a)} \frac{1}{d} \operatorname{acyc}(d, b),
$$

which gives the result.

Theorem 4.1. Assume $D$ is regular and $c_{a}(m ; D) \sim A_{a} \cdot B^{m}$ for $a \in G$. We have

$$
\tilde{c}_{a}(m ; D)=\frac{1}{m} A_{a} \cdot B^{m}\left(1+O\left(\omega^{m}\right)\right), \quad m \rightarrow \infty, 0 \leq \omega<1
$$

All but an exponentially small proportion of $\tilde{\mathcal{C}}_{a}(m ; D)$ and $\mathcal{C}_{a}(m ; D)$ are aperiodic. If $D$ satisfies the assumptions of Theorem 2.1, then $A_{a}$ does not depend on a.

Proof. From above we know

$$
\tilde{c}_{a}(m ; D)=\sum_{(d, b) \preceq(m, a)} \frac{1}{d} \operatorname{acyc}(d, b),
$$

where $\operatorname{acyc}(m, a)$ is the number of aperiodic cyclically restricted $m$-compositions of $a$. We claim that $\tilde{c}_{a}(m ; D) \sim \frac{1}{m} \operatorname{acyc}(m, a) \sim \frac{1}{m} c_{a}(m ; D)$. 
From Proposition 3.2 we have $c_{a}(m ; D)=A_{a} \cdot B^{m}\left(1+O\left(\theta^{m}\right)\right)$, where $B>1$. Now

$$
\begin{aligned}
\sum_{(d, b) \prec(m, a)} \frac{1}{d} \operatorname{acyc}(d, b) & \leq \sum_{(d, b) \prec(m, a)} \frac{1}{d} c_{b}(d ; D) \\
& \leq|G| \frac{m}{2} A_{a} \cdot B^{m / 2}\left(1+O\left(\max \left(B^{-m / 2}, \theta^{m / 2}\right)\right)\right) .
\end{aligned}
$$

On the other hand, we have

$$
\begin{aligned}
c_{a}(m ; D) \geq \operatorname{acyc}(m, a) & =c_{a}(m ; D)-\sum_{(d, b) \prec(m, a)} \operatorname{acyc}(d, b) \\
& \geq c_{a}(m ; D)-\sum_{(d, b) \prec(m, a)} c_{b}(d ; D),
\end{aligned}
$$

and so

$$
-\sum_{(d, b) \prec(m, a)} c_{b}(d ; D) \leq \operatorname{acyc}(m, a)-c_{a}(m ; D) \leq 0 .
$$

$\operatorname{Thus} \operatorname{acyc}(m, a)=A_{a} \cdot B^{m}\left(1+O\left(\omega^{m}\right)\right)$ where $\omega=\max \left(\theta, B^{-1 / 2}\right)$.

Theorem 4.2. Assume $U$ is nonempty and suppose $D=\bar{D}-U$ is regular with strongly connected derived digraph $D_{\times}$.

For $u \in V(\bar{D})$, let $\mu(u)$ be the minimum number of occurrences of $U$ in a composition in $\mathcal{P}\left(\bar{D},\{u\}, N^{-}(u)\right)$ with at least 1 occurrence of $V(D)$. Let $\mu$ be the minimal such $\mu(u)$. Assume for all sufficiently large values of $m$ there exist compositions in $\mathcal{P}(m ; \bar{D}, V(D), V(D))$ with exactly 1 occurrence of $U$, and that $p(m ; D, V(D), V(D)) \sim A \cdot B^{m}$.

If $r \geq \max (\mu, 1), \mu \geq 0$ then the number of circular $m$-compositions of $a \in G$ with exactly $r$ cyclic occurrences of $U$ is

$$
\tilde{c}_{a}(m, r ; D)=m^{r-\mu-1} A_{r, \mu} \cdot B^{m}\left(1+O\left(m^{-1}\right)\right), \quad m \rightarrow \infty .
$$


Proof. Let $Q=\mathbb{Z}_{>0} \times G \times \mathbb{Z}_{>0}$ be a poset where $\left(j_{1}, a, j_{2}\right) \preceq\left(k_{1}, b, k_{2}\right)$ if $j_{1} \mid k_{1}$, $\left(k_{1} / j_{1}\right) a=b$, and $\left(k_{1} / j_{1}\right) j_{2}=k_{2}$. The Moebius function $\mu_{Q}$ of $Q$ is defined recursively by $\mu_{Q}(s, s)=1$ for $s \in Q$ and $\mu_{Q}(s, u)=-\sum_{s \preceq t \prec u} \mu_{Q}(s, t)$ for $s \prec u$ in $Q$. By analogy to Proposition 4.1 we have

$$
\begin{aligned}
& \tilde{c}_{a}(m, r) \\
= & \sum_{\left(d_{1}, b, d_{2}\right) \preceq(m, a, r)} \frac{1}{d} \sum_{\left(d_{1}^{\prime}, b^{\prime}, d_{2}^{\prime}\right) \preceq\left(d_{1}, b, d_{2}\right)} c_{b^{\prime}}\left(d_{1}^{\prime}, d_{2}^{\prime} ; D\right) \mu_{Q}\left(\left(d_{1}^{\prime}, b^{\prime}, d_{2}^{\prime}\right),\left(d_{1}, b, d_{2}\right)\right) .
\end{aligned}
$$

Following the proof of Theorem 4.1, the dominant term is $m^{-1} c_{a}(m, r ; D)$, so we conclude with reference to Theorem 3.1.

Definition 4.1. A mixture of two random variables $X, Y$ with weights $0 \leq p, 1-p \leq 1$ is a random variable $Z$ such that the distribution functions satisfy $F_{Z}(x)=p F_{X}(x)+$ $(1-p) F_{Y}(x), x \in \mathbb{R}$

The following lemma is an expedient used to show when normalized convergence in distribution holds up to low-probability events.

Lemma 4.3. Let $X_{n}, Y_{n} \geq 0$ be $L^{2}$ random variables for $n \in \mathbb{Z}_{>0}$. Let $Z_{n}$ be a mixture of $X_{n}$ and $Y_{n}$ with weights $p_{n}$ and $1-p_{n}$, where $p_{n} \rightarrow 1$. Assume that $E\left(X_{n}\right)$ or $E\left(Z_{n}\right)$ are bounded away from 0 , and that $\operatorname{Var}\left(Z_{n}\right)$ or $\operatorname{Var}\left(X_{n}\right)$ are bounded away from 0 , and that

$$
\left(1-p_{n}\right)\left(E\left(Y_{n}^{2}\right)+E\left(Y_{n}\right) E\left(X_{n}\right)+E\left(X_{n}^{2}\right)\right)=o(1) .
$$

Then we have $\left(X_{n}-E\left(X_{n}\right)\right) / \sqrt{\operatorname{Var}\left(X_{n}\right)} \Rightarrow F$ if and only if $\left(Z_{n}-E\left(Z_{n}\right)\right) / \sqrt{\operatorname{Var}\left(Z_{n}\right)} \Rightarrow$ $F$, and $E\left(X_{n}\right) \sim E\left(Z_{n}\right), \operatorname{Var}\left(X_{n}\right) \sim \operatorname{Var}\left(Z_{n}\right)$. 
Proof. We have $E\left(Z_{n}\right)=p_{n} E\left(X_{n}\right)+\left(1-p_{n}\right) E\left(Y_{n}\right)$, and in general

$$
\begin{aligned}
E\left(Z_{n}^{2}\right) & =2 \int_{0}^{\infty} x P\left(Z_{n}>x\right) d x \\
& =2 \int_{0}^{\infty} x p_{n} P\left(X_{n}>x\right)+x\left(1-p_{n}\right) P\left(Y_{n}>x\right) d x \\
& =p_{n} E\left(X_{n}^{2}\right)+\left(1-p_{n}\right) E\left(Y_{n}^{2}\right)
\end{aligned}
$$

by [Res13, Ex. 22b]. Now

$$
\begin{aligned}
\operatorname{Var}\left(Z_{n}\right) & =E\left(Z_{n}^{2}\right)-E\left(Z_{n}\right)^{2} \\
& =p_{n} E\left(X_{n}^{2}\right)+\left(1-p_{n}\right) E\left(Y_{n}^{2}\right)-\left(p_{n} E\left(X_{n}\right)+\left(1-p_{n}\right) E\left(Y_{n}\right)\right)^{2} \\
& =p_{n} \operatorname{Var}\left(X_{n}\right)+\left(1-p_{n}\right) \operatorname{Var}\left(Y_{n}\right)+p_{n}\left(1-p_{n}\right)\left(E\left(X_{n}\right)-E\left(Y_{n}\right)\right)^{2} .
\end{aligned}
$$

From the assumptions we know $\left(1-p_{n}\right) \operatorname{Var}\left(Y_{n}\right) \leq\left(1-p_{n}\right) E\left(Y_{n}^{2}\right)=o(1)$. And

$$
\begin{aligned}
p_{n}\left(1-p_{n}\right)\left(E\left(X_{n}\right)-E\left(Y_{n}\right)\right)^{2} & \leq\left(1-p_{n}\right) 2\left(E\left(X_{n}\right)^{2}+E\left(Y_{n}\right) E\left(X_{n}\right)+E\left(Y_{n}\right)^{2}\right) \\
& \leq\left(1-p_{n}\right) 2\left(E\left(X_{n}^{2}\right)+E\left(Y_{n}\right) E\left(X_{n}\right)+E\left(Y_{n}^{2}\right)\right) \\
& =o(1) .
\end{aligned}
$$

Thus

$$
E\left(Z_{n}\right) \sim E\left(X_{n}\right) \text { and } \operatorname{Var}\left(Z_{n}\right) \sim \operatorname{Var}\left(X_{n}\right)
$$

By Theorem 2.4 theorem we have

$$
\frac{X_{n}-E\left(X_{n}\right)}{\sqrt{\operatorname{Var}\left(X_{n}\right)}} \Rightarrow F \text { iff } \frac{X_{n}-E\left(Z_{n}\right)}{\sqrt{\operatorname{Var}\left(Z_{n}\right)}} \Rightarrow F
$$

and

$$
\frac{Z_{n}-E\left(Z_{n}\right)}{\sqrt{\operatorname{Var}\left(Z_{n}\right)}} \Rightarrow F \text { iff } \frac{Z_{n}-E\left(X_{n}\right)}{\sqrt{\operatorname{Var}\left(X_{n}\right)}} \Rightarrow F
$$


If $\mathcal{C}(F) \subseteq \mathbb{R}$ is the set of points where $F$ is continuous, then for $x \in \mathcal{C}(F)$ we have

$$
\begin{aligned}
\lim _{n \rightarrow \infty} F_{Z_{n}}\left(\sqrt{\operatorname{Var}\left(Z_{n}\right)} x+E\left(Z_{n}\right)\right)= & \lim _{n \rightarrow \infty} p_{n} F_{X_{n}}\left(\sqrt{\operatorname{Var}\left(Z_{n}\right)} x+E\left(Z_{n}\right)\right) \\
& +\left(1-p_{n}\right) F_{Y_{n}}\left(\sqrt{\operatorname{Var}\left(Z_{n}\right)} x+E\left(Z_{n}\right)\right) \\
= & \lim _{n \rightarrow \infty} p_{n} F_{X_{n}}\left(\sqrt{\operatorname{Var}\left(Z_{n}\right)} x+E\left(Z_{n}\right)\right) \\
= & \lim _{n \rightarrow \infty} F_{X_{n}}\left(\sqrt{\operatorname{Var}\left(Z_{n}\right)} x+E\left(Z_{n}\right)\right) \\
= & \lim _{n \rightarrow \infty} F_{X_{n}}\left(\sqrt{\operatorname{Var}\left(X_{n}\right)} x+E\left(X_{n}\right)\right) .
\end{aligned}
$$

Theorem 4.3. Assume that $|G| \geq 2$ and that $U \subset \operatorname{Seq}_{\sigma}(G)$ is non-empty. Then the number of cyclic occurrences of $U$ in a uniform random circular $m$-composition of $a \in G$ is asymptotically normal with mean and variance asymptotic to those of the number of occurrences of $U$ in a uniform random word over $G$.

Proof. Let $X_{m}, X_{m}^{\langle a p\rangle}, X_{m}^{\langle a p, c\rangle}, X_{m}^{\langle c\rangle}$ be the number of cyclic occurrences of $U$ in a uniform random $m$-composition of $a$, aperiodic $m$-composition of $a$, aperiodic circular $m$-composition of $a$, and circular $m$-composition of $a$.

By Theorem 3.2 we have $\left(X_{m}-E\left(X_{m}\right)\right) / \operatorname{Var}\left(X_{m}\right) \Rightarrow N(0,1)$. The quantities $E\left(X_{m}\right)$ and $\operatorname{Var}\left(X_{m}\right)$ are asymptotically proportional to $m$ thus bounded away from 0 .

The number of occurrences in a uniform random (circular) composition is a mixture of the number of occurrences in a uniform random periodic (circular) composition and the number of occurrences in a uniform random aperiodic (circular) composition. The weights are simply the proportion of (circular) compositions that are periodic and aperiodic, respectively. By Theorem 4.1, the proportion of $m$-compositions, circular or not, that are periodic is exponentially small. There can be at most $m$ occurrences in an $m$-composition, so moments of the number of occurrences of $U$ in a (circular) 
$m$-composition are $m^{O(1)}$.

We are set up to apply Lemma 4.3 twice. The first application allows us to conclude that $X_{m}$ and $X_{m}^{\langle a p\rangle}$ have the same limiting distribution. The second gives that $X_{m}^{\langle c\rangle}$ and $X_{m}^{\langle a p, c\rangle}$ have the same limiting distribution. Clearly $X_{m}^{\langle a p\rangle}$ and $X_{m}^{\langle a p, c\rangle}$ have the same distributions for all $m$, so we are done.

Some examples of circular objects follow.

Example 4.1. For a composition $x=(x(1), \ldots, x(m))$, we define

$$
\operatorname{gap}(x)=\max _{i} x(i)-\min _{i} x(i)+1-|\{x(i): i=1,2, \ldots, m\}|,
$$

which is the number of parts missing between the minimum and maximum parts of $x$. If $\operatorname{gap}(x)=0$ we say $x$ is gap-free. Research Direction 3.1 parts (3) and (4) in $[$ HM10, p. 86] ask for an explicit generating function for the number of circular compositions/words $x$ such that $\operatorname{gap}(x)=\ell$.

Let $c(m)$ be the number of gap-free $k$-ary words and let $\tilde{c}(m)$ be the number of circular gap-free words. The number of gap-free $k$-ary words with $j$ distinct letters is $(k-j+1) j !\left\{\begin{array}{c}m \\ j\end{array}\right\}$. Thus

$$
c(m)=\sum_{j=1}^{k}(k-j+1) j !\left\{\begin{array}{c}
m \\
j
\end{array}\right\} \sim \sum_{j=1}^{k}(k-j+1) j^{m} \sim k^{m},
$$

where we apply the asymptotics of the Stirling subset numbers [Olv+17]. The first letter in a gap-free $m$-word is arbitrary if the remaining $(m-1)$-word has $k$ distinct letters. The number of such words is $k !\left\{\begin{array}{c}m-1 \\ k\end{array}\right\} \sim k^{m-1}$, so the first letter is arbitrary in almost all gap-free words. Thus for an abelian finite group $G$, the number of gap-free $m$-compositions of $a$ is $c_{a}(m) \sim k^{m-1}$. 
Using the familiar Moebius function $\mu$, as in [BG75], we have

$$
\begin{aligned}
\tilde{c}(m) & =\sum_{d \mid m} \frac{1}{d} \sum_{d^{\prime} \mid d} \mu\left(d / d^{\prime}\right) c\left(d^{\prime}\right) \\
& \sim \frac{1}{m} k^{m} .
\end{aligned}
$$

The number of circular gap-free $m$-compositions of $a \in G$ is $\tilde{c}_{a}(m) \sim \frac{1}{m} k^{m-1}$.

Example 4.2. Considering avoidance of the subword pattern 132, for any total $a$ there is 1 composition with 1 part, namely $(a)$. For $m \geq 2$, some compositions are grouped into non-trivial equivalence classes. For $m=1, \ldots, 5$, the numbers of 132 avoiding circular $m$-compositions of 0 over $\mathbb{Z}_{5}$ are $1,3,7,23,82$, and the counts for m-compositions of 1 are $1,3,7,23,77$.

\subsection{Note on counting palindromic compositions}

Notation 4.1. For a finite sequence $x=(x(1), \ldots, x(m))$, the reversed sequence is written $\overleftarrow{x}=(x(m), \ldots, x(1))$.

An unlabeled undirected weighted path of length $m$ restricted according to $D$ is equivalent to an unordered pair $\{x, \overleftarrow{x}\}$ where $x, \overleftarrow{x} \in \mathcal{P}(m ; D)$, or the singleton $\{x\}$ if $x \in \mathcal{P}(m ; D)$ and $x=\overleftarrow{x}$. These may also be called undirected words. For simplicity we assume all vertices of $D$ are allowed as start and finish vertices.

Proposition 4.2. Assume $D$ is such that $x \in \mathcal{P}(D) \Longrightarrow \stackrel{\leftarrow}{x} \in \mathcal{P}(D)$. Let $\Xi=\{\xi$ : $\overleftarrow{\xi \xi} \in \mathcal{P}(D), \xi \in V(D)\}$. If $m \geq 2 \sigma$ is even, the number of $G$-weighted undirected 
paths of length $m$ with total a restricted by $D$ is

$$
\tilde{p}_{a}(m ; D)=\frac{1}{2} p_{a}(m ; D)+\sum_{b: 2 b=a} \frac{1}{2} p_{b}(m / 2 ; D, V(D), \Xi) .
$$

For $c \in G$, let $\Xi_{c}=\{\xi: \xi c \overleftarrow{\xi} \in \mathcal{P}(D), \xi \in V(D)\}$. If $m \geq 2 \sigma$ is odd, the number of $G$-weighted undirected paths of length $m$ with total a restricted by $D$ is

$$
\tilde{p}_{a}(m ; D)=\frac{1}{2} p_{a}(m ; D)+\frac{1}{2} \sum_{c \in G} \sum_{b: 2 b+c=a} p_{b}\left((m-1) / 2 ; D, V(D), \Xi_{c}\right) .
$$

Proof. The number of undirected paths is determined by dividing by 2 , with an adjustment for palindromic compositions: those $x$ such that $x=\overleftarrow{x}$. If $m$ is even, the set of palindromic $m$-compositions is in correspondence with $\mathcal{P}(m / 2 ; D, V(D), \Xi)$ and

$$
\begin{aligned}
\tilde{p}_{a}(m ; D)= & \frac{1}{2}\left(p_{a}(m ; D)-\sum_{b: 2 b=a} p_{b}(m / 2 ; D, V(D), \Xi)\right) \\
& +\sum_{b: 2 b=a} p_{b}(m / 2 ; D, V(D), \Xi) .
\end{aligned}
$$

The case of even $m$ is similar.

The analogous result for integer compositions is found in [BC09, §11]. 


\section{Subsequence pattern avoidance}

Given a word $w$ over $[k]$, the reduction of $w$, written $\operatorname{red}(w)$, is obtained by replacing the $j^{\text {th }}$ smallest letters of $w$ with $j$ 's, for all $j$. For example, $\operatorname{red}(46632)=34421$. A subsequence pattern, sometimes called a classical pattern, is a word over some $[k]$ written with hyphens between letters: 1-1-1-1-3-2-2 $=1^{4}-3-2^{2} \in[3]^{7}=\operatorname{SEQ}_{7}([3])$. Given words $w$ of length $m$ and $\tau$ of length $l$, an occurrence of $\tau$, as a subsequence pattern, in $w$ is a sequence of indices $1 \leq i_{1}<\cdots<i_{l} \leq m$ such that $\operatorname{red}\left(w\left(i_{1}\right), \ldots, w\left(i_{l}\right)\right)=\tau$.

A partially ordered pattern is similar to a subsequence pattern except that not all letters are comparable. The letters in a partially ordered pattern are from a partially ordered alphabet; letters shown with the same number of primes are comparable to each other (e.g. $1^{\prime \prime}$ and $2^{\prime \prime}$ ), while letters shown without primes are comparable to all letters of the alphabet. An occurrence of a partially ordered pattern in a word $w$ is a distinguished subsequence of terms of $w$ such that the relative order of two entries in the subsequence need be the same as that of the corresponding letters in the pattern only if the corresponding letters in the pattern are comparable; e.g. the partially ordered pattern $1^{\prime}-1^{\prime \prime}-2$ is found in the word 42213 three times as $4 \underline{22} 1 \underline{3}$, $4 \underline{2213}$ and 42213 (the subsequences of length three in which the third letter is larger than the first two).

A generalized pattern is again similar to a subsequence pattern except there may or may not be a hyphen between adjacent letters. If there is no hyphen, those two letters can only match with adjacent letters in a word. For example, if $\tau=11-2$, then 424135 has no occurrences of $\tau$ but 244135 has the occurrence $2 \underline{4413} \underline{5}$. 
Subsequence patterns were first studied in the context of permutations [Sta07] but are now adapted to different objects. The number of $k$-ary words of length $m$ avoiding a given subsequence or generalized pattern has been studied for a number of different patterns [Bur98; Reg98; BM05; Man06; Pud08; FM09; JM09; HM10; MS15]. Specifically, exact results for the avoidance of various subsequence patterns with at most 2 distinct letters were found in [BM02]. For partially ordered pattern-based enumeration for words and other objects, see [KM03; Kit05; HKM07; BK08]. The article [GMP11] counts words with $r \geq 0$ occurrences of a some simple subsequence patterns.

Occurrences of subsequence, partially ordered, and generalized patterns are defined for compositions as they are for words. The counting question simply changes to, how many compositions with length $m$ and total $n$ avoid the pattern?

A generating function counting integer compositions avoiding some 3-letter patterns is given in the note [SW06], which is a simplification of earlier work in [ALW95]. A recurrence relation is also given in $[\mathrm{Alb}+01]$. Compositions avoiding the remaining 3letter patterns, and pairs of 3-letter patterns are counted in [HM06]. That paper also looks at the subsequence pattern $1^{p}-2-1^{q}$. Partially ordered patterns in compositions are considered in [HKM07]. Compositions avoiding a generalized pattern of length 3 are counted using generating functions in [HM10, § 5.3].

Remark 5.1. Let $p_{k}(m, r)$ be the number of $k$-ary $m$-words with $r$ occurrences of the pattern $1-\cdots-1=1^{p}$. A simple argument shows

$$
\sum_{m, r \geq 0} p_{k}(m, r) \frac{z^{m}}{m !} u^{r}=\left(\sum_{i \geq 0} u^{\left(\begin{array}{c}
i \\
p
\end{array}\right)} \frac{z^{i}}{i !}\right)^{k} .
$$

The recent paper [Sch17] sheds light on expressions of this form, by establishing 
integral representations such as

$$
\sum_{n \geq 0} g_{n} q^{n^{2}} z^{n}=\frac{1}{\sqrt{2 \pi}} \int_{0}^{\infty}\left(\sum_{b= \pm 1} G\left(e^{b t \sqrt{2 \log (q)}} z\right)\right) e^{-t^{2} / 2} d t
$$

where $G(z)=\sum_{n \geq 0} g_{n} z^{n}$. Enumerative applications of these representations have yet to be explored.

Remark 5.2. In the language of $\S 1$, we deal with paths avoiding global occurrences of digraph patterns. Undirected paths and directed and undirected cycles are approached in a similar manner.

For a weighted digraph $\Gamma$, let $s(\Gamma)$ be the symmetric closure of $\Gamma$, i.e. the underlying undirected graph. Given a weighted path $\Gamma_{p}$, and digraph pattern instance $P$ which is also a weighted path, an occurrence of $P$ in $s\left(\Gamma_{p}\right)$ is either an occurrence of $P$ in $\Gamma_{p}$ or an occurrence of $P^{-1}$ in $\Gamma_{p}$, where $P^{-1}$ is $P$ with $\operatorname{arcs}$ reversed. Let $c\left(\Gamma_{p}\right)$ be the directed cycle formed by adding an arc to $\Gamma_{p}$. Then an occurrence of $P$ in $c\left(\Gamma_{p}\right)$ is the occurrence of some circular shift of $P$ in $\Gamma_{p}$. Occurrences of $P$ in $s\left(c\left(\Gamma_{p}\right)\right)$ are occurrences of circular shifts and/or reversals of $P$ in $\Gamma_{p}$.

\subsection{Words and integer compositions}

This section fills some gaps in the literature on words and integer compositions that avoid a pattern. Our main tools are recurrence relations and generating functions, and we use various standard counting techniques.

Remark 5.3. The random sampling in this section is performed by exploiting the structure of recurrence relations. The method achieves exact uniform sampling and makes use of two rules, one for addition and one for multiplication. Assume there 
are three classes of objects, $A, B, C$ and the number of objects in each are $a, b, c$. We have the relation $a=b+c$ if $A=B \dot{\cup} C$. Then to draw an object uniformly randomly from $A$, we may draw an object from $B$ with probability $b /(b+c)$ or an object from $C$ with probability $c /(b+c)$. Now if $A=B \times C$, we have $a=b c$. Here we may draw uniformly at random from $A$ by independently drawing from both $B$ and $C$. This simple method is often applicable where we have a recurrence relation, in which case we recurse until reaching a base case.

\subsubsection{Pairs of generalized patterns of length 3}

While we do not consider every possible pair of generalized patterns of length 3 in this section, we give a number of representative examples. We expect similar techniques apply to most of the remaining such pattern pairs.

\section{The pair $\{11-2,12-3\}$}

We use the generating function $P_{k}(w \mid z, u)$ to enumerate integer compositions over $[k]$ starting with the subword $w$ and avoiding $\{11-2,12-3\}$, where $z$ marks the total and $u$ marks the length. We write $P_{k}(z, u)$ for $P_{k}(e \mid z, u)$ where $e$ is the sequence of length 0 , and we write $P(z, u)$ to refer to $\lim _{k \rightarrow \infty} P_{k}(z, u)$.

Proposition 5.1. We have

$$
P(z, u)=\frac{1}{1-u z} \prod_{i \geq 2}\left(1-u z^{i} \prod_{j=1}^{i-1}\left(1+u z^{j}\right)\right)^{-1} .
$$

Proof. We follow the proof of Theorem 5.21 [HM10] at least in spirit. 
Take a composition $x$ over $[k]$ that avoids $\{11-2,12-3\}$. Assume $x$ begins with the part $i$ (where $1 \leq i \leq k$ ). Then $x$ is either $i$ by itself or begins $(i, j, \ldots)$ for some part $j$. Now if $j<i$, then the first part of $x$ cannot be involved in an occurrence of the pattern set, so the composition $(j, x(3), \ldots, x(m))$ is arbitrary as long as it avoids the pattern set. On the other hand, if $j \geq i$, no later parts may be greater than $j$ so the composition $(x(3), \ldots, x(m))$ is an arbitrary composition over $[j]$ avoiding the pattern set. This gives, for $k \geq 1,1 \leq i \leq k$,

$$
\begin{aligned}
P_{k}(i \mid z, u) & =z^{i} u+\sum_{j=1}^{i-1} P_{k}(i j \mid z, u)+\sum_{j=i}^{k} P_{k}(i j \mid z, u) \\
& =z^{i} u+z^{i} u\left(\sum_{j=1}^{i-1} P_{k}(j \mid z, u)+\sum_{j=i}^{k} z^{j} u P_{j}(z, u)\right) .
\end{aligned}
$$

Define $G_{k}(i)=P_{k}(i \mid z, u)-P_{k-1}(i \mid z, u)$ for $k \geq 2$ and $1 \leq i<k$. By Equation (5.1) we have $G_{k}(i)=z^{i} u\left(\sum_{j=1}^{i-1} G_{k}(j)+z^{k} u P_{k}(z, u)\right)$ for $1 \leq i<k$. It can then be seen by induction that $G_{k}(i)=u^{2} z^{i+k} P_{k}(z, u) \prod_{j=1}^{i-1}\left(1+u z^{j}\right), 1 \leq i<k$. We naturally define $G_{k}(k)=u z^{k} P_{k}(z, u)$. Induction or a combinatorial argument also show that for $k \geq 2$ we have

$$
\begin{aligned}
P_{k}(z, u)-P_{k-1}(z, u) & =\sum_{i=1}^{k-1} G_{k}(i)+G_{k}(k) \\
& =u z^{k} P_{k}(z, u) \sum_{i=1}^{k-1} u z^{i} \prod_{j=1}^{i-1}\left(1+u z^{j}\right)+u z^{k} P_{k}(z, u) \\
& =u z^{k} P_{k}(z, u)\left(-1+\prod_{j=1}^{k-1}\left(1+u z^{j}\right)\right)+u z^{k} P_{k}(z, u) \\
& =u z^{k} P_{k}(z, u) \prod_{j=1}^{k-1}\left(1+u z^{j}\right),
\end{aligned}
$$

so

$$
P_{k}(z, u)=\left(1-u z^{k} \prod_{j=1}^{k-1}\left(1+u z^{j}\right)\right)^{-1} P_{k-1}(z, u) .
$$




\begin{tabular}{|c|c|c|c|c|c|c|c|c|c|c|c|}
\hline$n \quad m$ & 0 & 1 & 2 & 3 & 4 & 5 & 6 & 7 & 8 & 9 & 10 \\
\hline 0 & 1 & 0 & 0 & 0 & 0 & 0 & 0 & 0 & 0 & 0 & 0 \\
\hline 1 & 0 & 1 & 0 & 0 & 0 & 0 & 0 & 0 & 0 & 0 & 0 \\
\hline 2 & 0 & 1 & 1 & 0 & 0 & 0 & 0 & 0 & 0 & 0 & 0 \\
\hline 3 & 0 & 1 & 2 & 1 & 0 & 0 & 0 & 0 & 0 & 0 & 0 \\
\hline 4 & 0 & 1 & 3 & 2 & 1 & 0 & 0 & 0 & 0 & 0 & 0 \\
\hline 5 & 0 & 1 & 4 & 5 & 2 & 1 & 0 & 0 & 0 & 0 & 0 \\
\hline 6 & 0 & 1 & 5 & 8 & 6 & 2 & 1 & 0 & 0 & 0 & 0 \\
\hline 7 & 0 & 1 & 6 & 12 & 12 & 6 & 2 & 1 & 0 & 0 & 0 \\
\hline 8 & 0 & 1 & 7 & 17 & 20 & 15 & 6 & 2 & 1 & 0 & 0 \\
\hline 9 & 0 & 1 & 8 & 23 & 33 & 28 & 16 & 6 & 2 & 1 & 0 \\
\hline 10 & 0 & 1 & 9 & 29 & 50 & 50 & 35 & 16 & 6 & 2 & 1 \\
\hline
\end{tabular}

Table 5: Counts of the $m$-compositions of $n$ avoiding $\{12-2,12-3\}$.

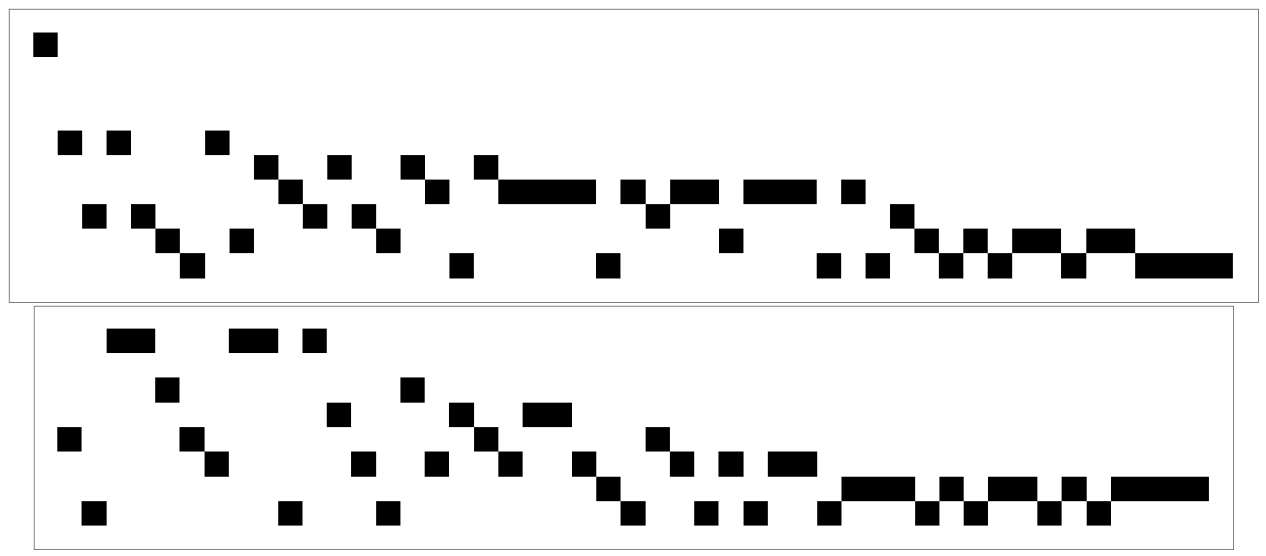

Figure 18: Uniform-randomly generated compositions of 150 avoiding $\{12-2,12-3\}$.

With the initial condition $P_{1}(z, u)=1 /(1-u z)$ we have

$$
P_{k}(z, u)=\frac{1}{1-u z} \prod_{i=2}^{k}\left(1-u z^{i} \prod_{j=1}^{i-1}\left(1+u z^{j}\right)\right)^{-1}
$$

We conclude the result by letting $k \rightarrow \infty$.

Figure 18 shows randomly generated compositions avoiding $\{12-2,12-3\}$. Table 5 show initial counts. 
The pair $\{21-2,2-12\}$

We count $k$-ary words avoiding the set of generalized patterns $\{21-2,2-12\}$. Note that it is not true that letters 1 must be found only in contiguous blocks at the very beginning and/or very end of a word. For example, 312 avoids the patterns and has the least letter in the middle.

Let $p_{k}(m)$ be the number of $k$-ary $m$-words that avoid $\{21-2,2-12\}$.

Proposition 5.2. For $k \geq 1$ we have

$$
p_{k}(m)=\frac{2^{1-k}}{(k-1) !} m^{2 k-2}+O\left(m^{2 k-3}\right), \quad m \rightarrow \infty .
$$

Proof. Take a $k$-ary word $w$ avoiding $\{21-2,2-12\}$. There are $p_{k-1}(m)$ such words with no letters $k$. We assume the greatest letter present in $w$ is $k$. All copies of $k$ must be contiguous in order to avoid the patterns. If we delete these copies of $k$ from $w$, the remaining word has the same structure but is a word over $[k-1]$. If there are $b$ letters $k$, there are $m-b+1$ possible positions of the contiguous run of these letters. Thus

$$
p_{k}(m)=p_{k-1}(m)+\sum_{b=1}^{m}(m-b+1) p_{k-1}(m-b), \quad k \geq 1, m \geq 0,
$$

and $p_{0}(m)=[m=0]$. Passing to the generating function $P_{k}(z)=\sum_{m \geq 0} p_{k}(m) z^{m}$ gives

$$
P_{k}(z)=P_{k-1}(z)+\frac{z}{1-z} D_{z}\left(z P_{k-1}(z)\right), P_{0}(z)=1 .
$$

By induction for $k \geq 1, P_{k}(z)$ has a unique singularity at 1 where it has a pole of 


\begin{tabular}{|rr|rrrrrrrrrrr|}
\hline$k$ & & 0 & 1 & 2 & 3 & 4 & 5 & 6 & 7 & 8 & 9 & 10 \\
\hline 2 & 1 & 2 & 4 & 7 & 11 & 16 & 22 & 29 & 37 & 46 & 56 \\
3 & 1 & 3 & 9 & 24 & 56 & 116 & 218 & 379 & 619 & 961 & 1431 \\
4 & 1 & 4 & 16 & 58 & 186 & 526 & 1324 & 3011 & 6283 & 12196 & 22276 \\
\hline
\end{tabular}

Table 6: Counts of the $k$-ary $m$-words avoiding $\{21-2,2-12\}$.

order $2 k-1$ and

$$
P_{k}(z)=\frac{\prod_{i=1}^{k-1}(2 i-1)}{(1-z)^{2 k-1}}+O\left((1-z)^{-(2 k-2)}\right), \quad z \rightarrow 1
$$

Using Theorem 2.2 we extract asymptotics for the coefficients of $P_{k}(z)$ to obtain

$$
p_{k}(m) \sim \frac{\prod_{i=1}^{k-1}(2 i-1)}{(2 k-2) !} m^{2 k-2} \text {. }
$$

We have

$$
\frac{\prod_{i=1}^{k-1}(2 i-1)}{(2 k-2) !}=\frac{2 k-3}{(2 k-2)(2 k-3)} \frac{\prod_{i=1}^{k-2}(2 i-1)}{(2(k-1)-2) !}=\frac{2^{1-k}}{(k-1) !}
$$

so we may conclude the result.

Table 6 gives initial counts of words avoiding this pattern set $\{21-2,2-12\}$, and Figure 19 has randomly generated examples.

\section{The pair $\{11-2,12-1\}$}

Let $P_{A}(w \mid z, u)$ be the generating function for integer compositions over the finite set $A \subset \mathbb{Z}_{>0}$, starting with the subword $w$, that avoid the pattern set $\{11-2,12-1\}$, where $z$ marks total and $u$ marks length. The generating function $P_{A}(z, u)$ refers to $P_{A}(e \mid z, u)$ where $e$ is the empty word. We use the notation $M(A, i)=\{j: j \in A, j \leq i\}$. 


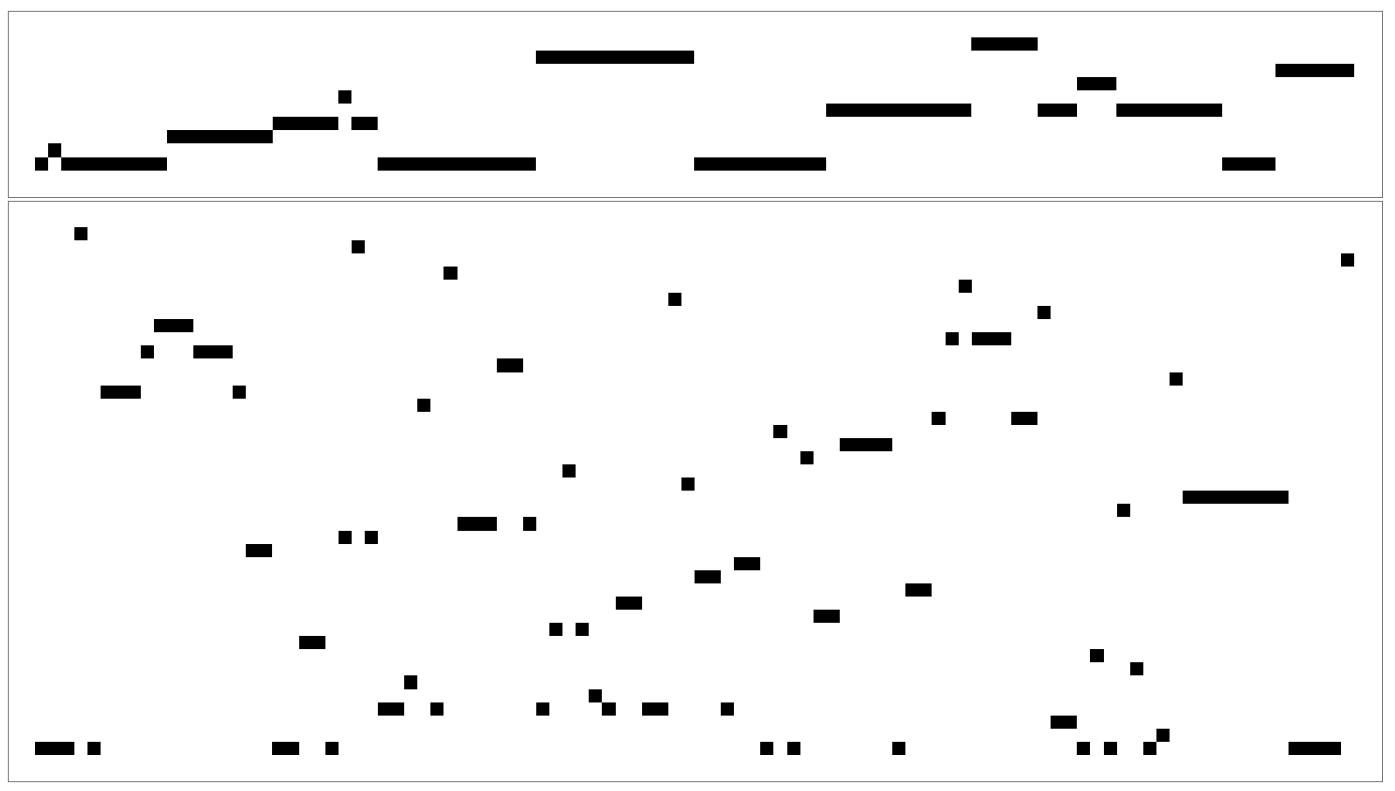

Figure 19: Uniform-randomly generated 10-ary (above) and 40-ary (below) 100-words avoiding $\{21-2,2-12\}$.

Proposition 5.3. We have

$$
P_{A}(z, u)=1+\sum_{i \in A} P_{A}(i \mid z, u)
$$

and

$$
\begin{aligned}
P_{A}(i \mid z, u)=z^{i} u & +\sum_{j \in A, j<i} z^{i} u P_{A}(j \mid z, u)+z^{2 i} u^{2} P_{M(A, i)}(z, u) \\
& +\sum_{j \in A, j>i} z^{i} u P_{A \backslash\{i\}}(j \mid z, u) .
\end{aligned}
$$

Proof. Let $x$ be a composition over $A$ avoiding $\{11-2,12-1\}$. If $x$ begins with the part $i \in A$, either $x=(i)$ or $x=(i, j, \ldots)$. In the latter case we may have $j<i, j=i$, or $j>i$, so we get

$$
P_{A}(i \mid z, u)=z^{i} u+\sum_{j \in A, j<i} P_{A}(i j \mid z, u)+P_{A}(i i \mid z, u)+\sum_{j \in A, j>i} P_{A}(i j \mid z, u) .
$$

If $j<i$ then $i$ is part of an occurrence only if $j$ is. So if we delete $i$ the remaining 


\begin{tabular}{|c|c|c|c|c|c|c|c|c|c|c|c|}
\hline$n \quad m$ & 0 & 1 & 2 & 3 & 4 & 5 & 6 & 7 & 8 & 9 & 10 \\
\hline 0 & 1 & 0 & 0 & 0 & 0 & 0 & 0 & 0 & 0 & 0 & 0 \\
\hline 1 & 0 & 1 & 0 & 0 & 0 & 0 & 0 & 0 & 0 & 0 & 0 \\
\hline 2 & 0 & 1 & 1 & 0 & 0 & 0 & 0 & 0 & 0 & 0 & 0 \\
\hline 3 & 0 & 1 & 2 & 1 & 0 & 0 & 0 & 0 & 0 & 0 & 0 \\
\hline 4 & 0 & 1 & 3 & 1 & 1 & 0 & 0 & 0 & 0 & 0 & 0 \\
\hline 5 & 0 & 1 & 4 & 4 & 1 & 1 & 0 & 0 & 0 & 0 & 0 \\
\hline 6 & 0 & 1 & 5 & 8 & 2 & 1 & 1 & 0 & 0 & 0 & 0 \\
\hline 7 & 0 & 1 & 6 & 11 & 7 & 2 & 1 & 1 & 0 & 0 & 0 \\
\hline 8 & 0 & 1 & 7 & 17 & 11 & 4 & 2 & 1 & 1 & 0 & 0 \\
\hline 9 & 0 & 1 & 8 & 24 & 24 & 10 & 4 & 2 & 1 & 1 & 0 \\
\hline 10 & 0 & 1 & 9 & 30 & 42 & 16 & 6 & 4 & 2 & 1 & 1 \\
\hline
\end{tabular}

Table 7: Counts of the $m$-compositions of $n$ avoiding $\{11-2,12-1\}$.

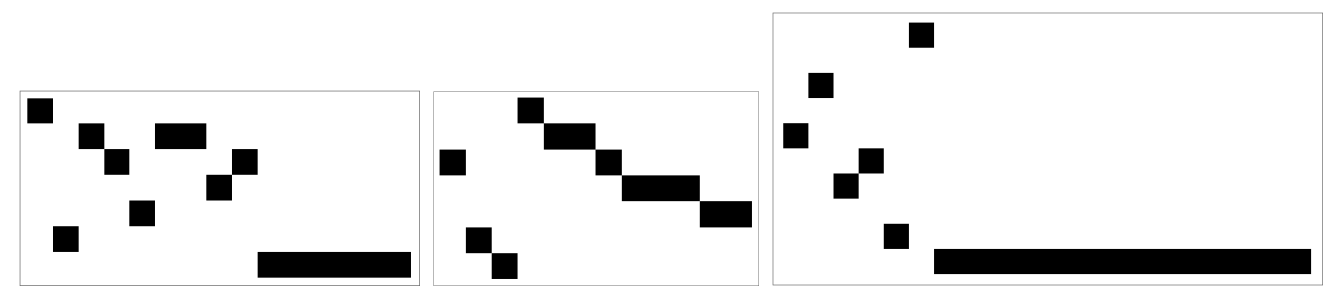

Figure 20: Uniform-randomly generated compositions of 50 avoiding $\{11-2,12-1\}$.

composition is arbitrary. If $j=i$ then $x=(i, i, \ldots)$. In order to avoid 11-2 the composition remaining after deleting $i i$ is arbitrary as long as no parts are above $i$. Finally if $j>i$ we may delete $i$ and have an arbitrary composition starting with $j$ as long as the part $i$ does not appear. Thus we have

$$
\begin{aligned}
z^{i} u+ & \sum_{j \in A, j<i} P_{A}(i j \mid z, u)+P_{A}(i i \mid z, u)+\sum_{j \in A, j>i} P_{A}(i j \mid z, u) \\
=z^{i} u & +\sum_{j \in A, j<i} z^{i} u P_{A}(j \mid z, u)+z^{2 i} u^{2} P_{M(A, i)}(z, u) \\
& +\sum_{j \in A, j>i} z^{i} u P_{A \backslash\{i\}}(j \mid z, u) .
\end{aligned}
$$

Table 7 shows initial counts of compositions avoiding $\{11-2,12-1\}$, and Figure 20 shows randomly-generated objects. 
The pair $\{12-3,3-21\}$

Let $p_{k}(m)$ be the number of $k$-ary $m$-words that avoid the pattern set $\{12-3,3-21\}$.

Proposition 5.4. For $k \geq 2$ we have $p_{k}(m) \sim A_{k} \cdot(\sqrt{k-1}+1)^{m}, m \rightarrow \infty$.

Proof. Let $w$ be a $k$-ary word avoiding $\{12-3,3-21\}$. Then either $w$ contains no letters $k$, or $w$ can be written as the concatenation

$$
w^{\prime} k^{j_{1}} w_{1} \cdots k^{j_{r}} w_{r} k^{s} w^{\prime \prime}
$$

where $r \geq 0, w^{\prime}$ is a word over $[k-1]$ that avoids $\{12,3-21\}, w^{\prime \prime}$ is a word on $[k-1]$ that avoids $\{21,12-3\}$, and the $w_{i}$ are words on $[k-1]$ that avoid $\{12,21\}$.

Nonempty words avoiding $\{12,21\}$ clearly have one distinct letter repeated some number of times. Words avoiding $\{21,12-3\}$ are either empty, have one distinct letter, or have exactly one increase and no decreases.

This translates to

$$
P_{k}(z)=P_{k-1}(z)+G_{k-1}(z) \frac{1}{1-(z /(1-z)) H_{k-1}(z)} \frac{z}{1-z} G_{k-1}(z),
$$

where $G_{k}(z)$ counts words avoiding $\{21,12-3\}$ (or $\{12,3-21\}$ ), so

$$
G_{k}(z)=1+\sum_{i=1}^{k} \frac{z}{1-z}+\sum_{i=1}^{k-1} \frac{z}{1-z} \sum_{j=i+1}^{k} \frac{z}{1-z}=1+k \frac{z}{1-z}+\frac{k^{2}-k}{2} \frac{z^{2}}{(1-z)^{2}},
$$

and $H_{k}(z)=k z /(1-z)$ counts nonempty words avoiding $\{12,21\}$. 


\begin{tabular}{|c|c|c|c|c|c|c|c|c|c|c|}
\hline$k^{m}$ & 0 & 1 & 2 & 3 & 4 & 5 & 6 & 7 & 8 & 9 \\
\hline 3 & 1 & 3 & 9 & 25 & 65 & 162 & 394 & 946 & 2258 & 5379 \\
\hline 4 & 1 & 4 & 16 & 56 & 174 & 502 & 138 & 3755 & 10059 & 26857 \\
\hline 5 & 1 & 5 & 25 & 105 & 375 & 1211 & 3689 & 10920 & 31920 & 92930 \\
\hline
\end{tabular}

Table 8: Counts of $k$-ary $m$-words avoiding $\{12-3,3-21\}$.

Iterating the recurrence relation, we have

$$
P_{k}(z)=\frac{1}{1-z}+\sum_{j=2}^{k} G_{j-1}^{2}(z) \frac{z}{1-z} \frac{1}{1-H_{j-1}(z) z /(1-z)} .
$$

We examine the factor

$$
\frac{1}{1-H_{j-1} z /(1-z)}=\frac{-z^{2}+2 z-1}{j z^{2}-2 z^{2}+2 z-1}
$$

The root of $j z^{2}-2 z^{2}+2 z-1$ with smallest absolute value is $z=\frac{1}{\sqrt{j-1}+1}$. For $j \geq 2$, this value is a simple pole less than 1 and decreasing (toward 0). By Theorem 2.2 we conclude the statement.

Table 8 shows initial coefficients of $P_{k}(z)$.

\subsubsection{Some partially ordered patterns with 2 letters}

Here we consider the family of partially ordered patterns of the form $2^{p}-1^{\prime}-\cdots-1^{(q)}-2^{r}=$ $2-\cdots-2-1^{\prime}-1^{\prime \prime}-\cdots-1^{(q)}-2-\cdots-2$. We break into cases depending on the values of $p, q, r$.

Case $p, q, r \geq 1$

Let $h_{k}(n, m)$ be the number of integer $m$-compositions of $n$ over $[k]$ that avoid $2^{p}-1^{\prime}-\cdots-1^{(q)}-2^{r}$ where $p, q, r \geq 1$. 
Proposition 5.5. We have the recurrence relation

$$
\begin{aligned}
& h_{k}(n, m) \\
& =\sum_{b=0}^{m}[0 \leq b<p+r \text { or } b>m-q]\left(\begin{array}{c}
m \\
b
\end{array}\right) h_{k-1}(n-b k, m-b) \\
& \quad+\sum_{b=p+r}^{m-q} \sum_{t=M}^{M+q-1}\left(\begin{array}{c}
w-2 \\
M-2
\end{array}\right)\left(\begin{array}{c}
m-t+1 \\
(p-1)+(r-1)+1
\end{array}\right) h_{k-1}(n-b k, m-b),
\end{aligned}
$$

for $m, n, \geq 0$ and $k \geq 2$. For $k=1$, we have $h_{1}(n, m)=[n=m]$.

Proof. Assume $k \geq 2$ and let $b$ be the number of letters $k$ in a word $w$. If $b \leq p+r-1$ or $b \geq m-q+1$, these letters cannot be part of an occurrence, so their positions do not matter, thus there are $\left(\begin{array}{l}n \\ b\end{array}\right) h_{k-1}(n-b k, m-b)$ such words $w$. If $b \geq p+r$, then between the $p^{\text {th }} k$ from the left and the $r^{\text {th }} k$ from the right there must be at most $q-1$ letters that are not $k$. Let $t$ be the number of all letters between the $p^{\text {th }} k$ from the left and the $r^{\text {th }} k$ from the right, and let $M=b-(p-1)-(r-1)$ be the number of letters $k$ among those letters. Then there are

$$
\sum_{t=M}^{M+q-1}\left(\begin{array}{c}
t-2 \\
M-2
\end{array}\right)\left(\begin{array}{c}
m-t+1 \\
(p-1)+(r-1)+1
\end{array}\right)
$$

possible ways of placing the letters $k$ in $w$. The first binomial coefficient chooses the letters $k$ between the $p^{\text {th }}$ from left and $r^{\text {th }}$ from right, and the second chooses the position of the remaining letters $k$ as well as the position of the $p^{\text {th }}$ from the left.

So for $m \geq p+r$, this gives

$$
\begin{aligned}
& h_{k}(n, m) \\
& =\sum_{b=0}^{m}[0 \leq b<p+r \text { or } b>m-q]\left(\begin{array}{c}
m \\
b
\end{array}\right) h_{k-1}(n-b k, m-b) \\
& \quad+\sum_{b=p+r}^{m-q} \sum_{t=M}^{M+q-1}\left(\begin{array}{c}
t-2 \\
M-2
\end{array}\right)\left(\begin{array}{c}
m-t+1 \\
(p-1)+(r-1)+1
\end{array}\right) h_{k-1}(n-b k, m-b),
\end{aligned}
$$


as desired. It can be verified that the recurrence is valid as well for the values $0 \leq m<p+r$.

We note that $h_{k}(n, m)$ is a function of $p+r$ rather than $p$ and $r$ independently.

Case $p=1, q=2, r=1$

For the special case $\tau=2-1^{\prime}-1^{\prime \prime}-2$ we illustrate an asymptotic analysis. We further simplify by ignoring totals and counting words. Let $H_{k}(z)=\sum_{m \geq 0} h_{k}(m) z^{m}$ where $h_{k}(m)$ is the number of $k$-ary $m$-words that avoid $2-1^{\prime}-1^{\prime \prime}-2$.

Proposition 5.6. If $k \geq 2$ we have $h_{k}(m)=\frac{A_{k}}{(3(k-1)) !} m^{3(k-1)}\left(1+O\left(m^{-1}\right)\right), m \rightarrow \infty$, where $A_{k}=\prod_{j=1}^{k-1}(1+3(j-1))$.

Proof. By Proposition 5.5 we know

$$
\begin{aligned}
h_{k}(m)= & h_{k-1}(m)+m h_{k-1}(m-1) \\
& \quad+\sum_{b=2}^{m-2}((m-b+1)+(m-b)(b-1)) h_{k-1}(m-b) \\
& \quad+m h_{k-1}(1)+h_{k-1}(0) \\
= & \sum_{b=0}^{m}((m-b+1)+(m-b)(b-1)) h_{k-1}(m-b) .
\end{aligned}
$$

Passing to generating functions, we have

$$
H_{k}(z)=\frac{1}{1-z} H_{k-1}(z)+\frac{z^{2}}{(1-z)^{2}} H_{k-1}^{\prime}(z)
$$

for $k \geq 2$, and $H_{1}(z)=1 /(1-z)$.

By induction $H_{k}(z)$ is rational with unique singularity at $z=1$ and $H_{k}(z) \sim$ 


\begin{tabular}{|c|c|c|c|c|c|c|c|c|c|c|}
\hline$k^{m}$ & 0 & 1 & 2 & 3 & 4 & 5 & 6 & 7 & 8 & 9 \\
\hline 1 & 1 & 1 & 1 & 1 & 1 & 1 & 1 & 1 & 1 & 1 \\
\hline 2 & 1 & 2 & 4 & 8 & 15 & 26 & 42 & 64 & 93 & 130 \\
\hline 3 & 1 & 3 & 9 & 27 & 76 & 196 & 462 & 1002 & 2019 & 3817 \\
\hline 4 & 1 & 4 & 16 & 64 & 242 & 844 & 2692 & 7852 & 21043 & 52184 \\
\hline 5 & 1 & 5 & 25 & 125 & 595 & 2635 & 10743 & 40163 & 137738 & 434798 \\
\hline
\end{tabular}

Table 9: Counts of the $k$-ary words of length $m$ avoiding $2-1^{\prime}-1^{\prime \prime}-2$.

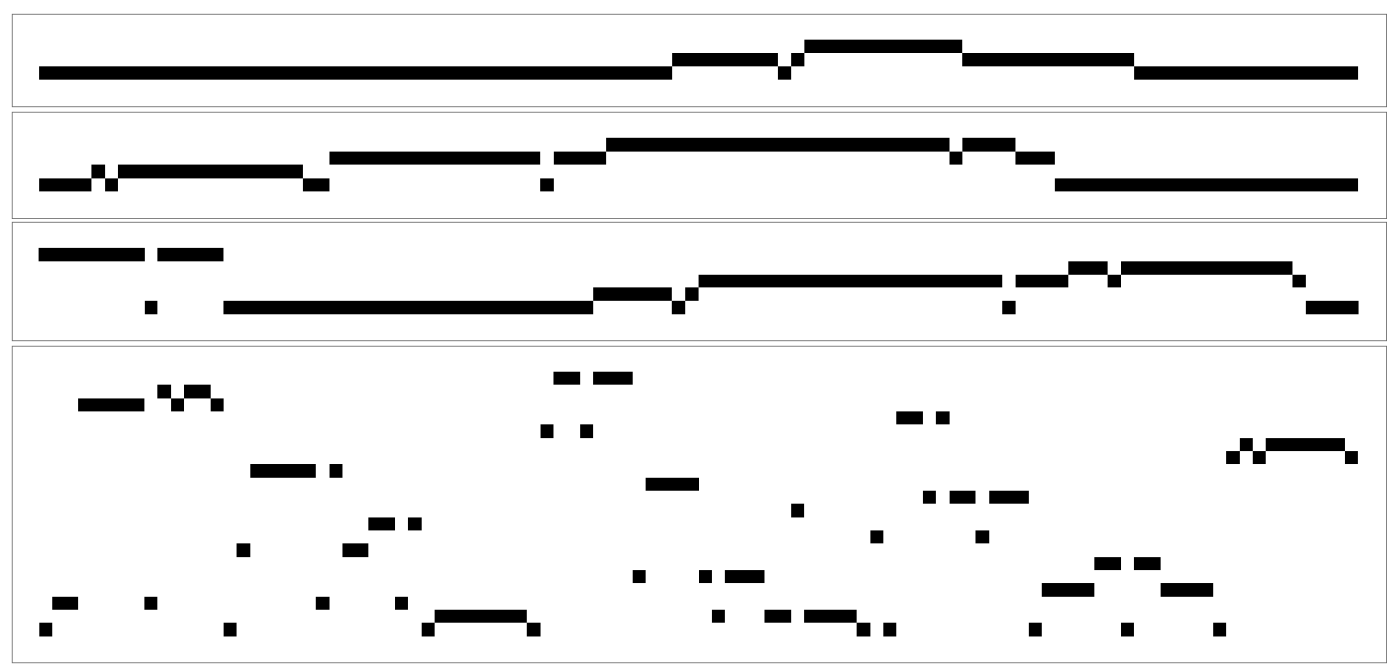

Figure 21: Uniform-randomly generated $k$-ary 100-words where $k=3,4,5,20$ (top to bottom) avoiding $2-1^{\prime}-1^{\prime \prime}-2$.

$A_{k} \frac{1}{(1-z)^{1+3(k-1)}}, z \rightarrow 1$ so by Theorem 2.2 we conclude the statement.

Table 9 shows initial coefficients of $H_{k}(z)$. Figure 21 has uniform-randomly generated words avoiding $2-1^{\prime}-1^{\prime \prime}-2$.

Case $p=0$

If we have $q, r \geq 1$ but we allow $p=0$, we have the pattern $\tau=1^{\prime}-1^{\prime \prime}-\cdots-1^{(q)}-2^{r}$. Words avoiding $\tau$ were counted in [GMP11, §2]; integer compositions were left as an open problem. Let $h_{k}(n, m)$ be the number of integer $m$-compositions of $n$ over $[k]$ that avoid $\tau$. 
Proposition 5.7. For $k \geq 2$ and $m \geq q+r$ we have

$$
\begin{aligned}
h_{k}(n, m)= & \sum_{j=1}^{q}\left(\begin{array}{l}
q \\
j
\end{array}\right) h_{k}(n-j k, m-j)(-1)^{j+1} \\
& +\sum_{b=0}^{r-1}\left(\begin{array}{c}
m-q \\
b
\end{array}\right) h_{k-1}(n-b k, m-b),
\end{aligned}
$$

and $h_{1}(n, m)=[n=m]$.

Proof. For the range $m \geq q+r$, we recursively count $m$-compositions $x$ avoiding $\tau$ by first counting $x$ such that at least one of the first $q$ letters is $k$. By the principle of inclusion-exclusion, the number of such $x$ is

$$
\sum_{j=1}^{q} N_{j}(-1)^{j+1}
$$

where $N_{j}$ is the sum, over all $j$-subsets of the first $q$ positions, of the number of compositions $x$ with $k$ 's in the positions given by the subset. The quantity $N_{j}$ is given by

$$
N_{j}=\left(\begin{array}{l}
q \\
j
\end{array}\right) h_{k}(n-j k, m-j),
$$

since inserting $j$ copies of $k$ into any of the first $q$ positions of an $(m-j)$-composition is reversible and does not affect the number of occurrences of $\tau$.

Now we count the compositions $x$ that have no letters $k$ in their first $q$ positions. Let $b$ be the number of letters $k$ in $x$. If $b \leq r-1$, then there are not enough letters $k$ to be part of a pattern, so there are

$$
\sum_{b=0}^{r-1}\left(\begin{array}{c}
m-q \\
b
\end{array}\right) h_{k-1}(n-b k, m-b),
$$

compositions of this kind. 


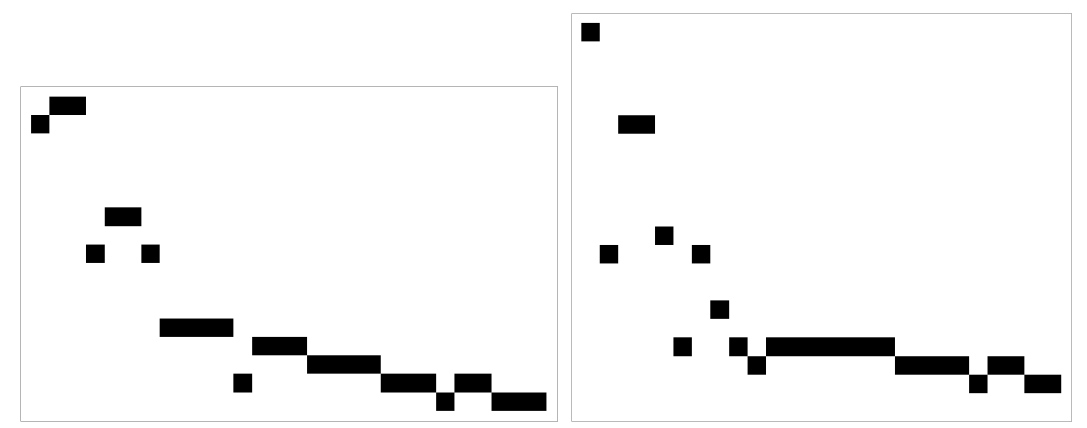

Figure 22: Uniform-randomly generated compositions of 150 avoiding $1^{\prime}-1^{\prime \prime}-2$.

If $b \geq r$ then there is at least one occurrence of $\tau$. Thus we have, for $m \geq q+r, k \geq 2$,

$$
\begin{aligned}
h_{k}(n, m)=\sum_{j=1}^{q} & \left(\begin{array}{l}
q \\
j
\end{array}\right) h_{k}(n-j k, m-j)(-1)^{j+1} \\
& +\sum_{b=0}^{r-1}\left(\begin{array}{c}
m-q \\
b
\end{array}\right) h_{k-1}(n-b k, m-b) .
\end{aligned}
$$

For $m<q+r$, we have $h_{k}(n, m)=\left[z^{n} u^{m}\right]\left(\sum_{j=1}^{k} z^{j} u\right)^{m}$.

We expect similar techniques to those used for $p, q, r \geq 1$ and $p=0$ apply to count avoidance of $\tau$ where $\tau$ involves the letter 2 and mutually incomparable symbols $1^{(j)}$

Figure 22 shows randomly generated compositions avoiding $1^{\prime}-1^{\prime \prime}-2$.

\subsubsection{Note on counting with symmetries}

\section{Reversal (unlabeled undirected paths)}

As in $\S 4.2$ we say that an undirected word is an unordered pair $\{w, \overleftarrow{w}\}$ where $w \neq \overleftarrow{w}$ or simply $\{w\}$ if $w=\overleftarrow{w}$. The pair $\{w, \overleftarrow{w}\}$ avoids a pattern $\tau$ if and only if both $w$ and $\overleftarrow{w}$ avoid $\tau$. For a subsequence pattern $\tau$, we define the set folds $(\tau)$ to be all possible 
words $\tau^{\prime}$ obtained by the following procedure. Split $\tau$ into two subwords $\tau=\tau_{1} \tau_{2}$. Take words $\tau^{\prime}$ such that $\tau_{1}$ and $\overleftarrow{\tau}_{2}$ are subsequences of $\tau^{\prime}$.

Proposition 5.8. Let $\tilde{p}_{k}(m ; T)$ be the number of undirected $k$-ary $m$-words that avoid all subsequence patterns in the set $T$. Then for even $m$ we have

$$
\tilde{p}_{k}(m ;\{\tau\})=\frac{1}{2} p_{k}(m ;\{\tau, \stackrel{\leftarrow}{\tau}\})+\frac{1}{2} p_{k}(m / 2 ; \text { folds }(\tau))
$$

Proof. An undirected word is either palindromic or not. If not, it corresponds to a pair of 2 distinct directed words. If it is palindromic, it takes the form $u \overleftarrow{u}$ where $u$ avoids folds $(\tau)$. Also,

$$
\tilde{p}_{k}(m ;\{\tau\})=\frac{1}{2}\left(p_{k}(m ;\{\tau, \stackrel{\leftarrow}{\tau}\})-p_{k}(m / 2 ; \text { folds }(\tau))\right)+p_{k}(m / 2 ; \text { folds }(\tau))
$$

The case of odd $m$ is less straightforward. Burstein [Bur98] counts a number of examples of words avoiding the set $\{\tau, \stackrel{\leftarrow}{\tau}\}$ for where $\tau$ is a short subsequence pattern with no repeated letters. Avoiding folds $(\tau)$ becomes quite restrictive but is not necessarily impossible. Any non-decreasing word avoids folds(2-1-3).

\section{Circular shift (unlabeled cycles)}

A circular word is an equivalence class of words where two words are equivalent if one is a circular shift of the other.

As in Remark 5.2, a word $w$ cyclically avoids a subsequence pattern $\tau$ if all circular shifts of $w$ avoid the pattern. Alternatively, $w$ cyclically avoids $\tau$ if $w$ avoids all circular shifts of $\tau$. We observe that we do not have property that if $u$ cyclically avoids a pattern so does $u$. For example, to cyclically avoid 1-2-3, we avoid the set 
$T=\{1-2-3,3-1-2,2-3-1\}$, and if $u=321$, then $u$ avoids the pattern but $u u=321321$ contains 2-3-1.

We define the set merges $_{i}(\tau)$ to contain all $\tau^{\prime}$ produced by the following procedure. If $i$ is an integer satisfying $1 \leq i<|\tau|$, we consider any circular shift $\tau^{*}$ of $\tau$ expressed as a concatenation of subwords $\tau^{*}=\tau_{1} \cdots \tau_{i}$, some of which may be empty. For each $\tau^{*}$, include any word $\tau^{\prime}$ such that each $\tau_{j}$ is a subsequence of $\tau^{\prime}$.

Proposition 5.9. Let $p_{k}(m ; T)$ be the number of $k$-ary $m$-words avoiding subsequence patterns in $T$, and let $\tilde{c}_{k}(m ; T)$ be the same for circular $m$-words. Let $t$ be the number of distinct letters in the subsequence pattern $\tau$. We have

$$
\begin{aligned}
& \tilde{c}_{k}(m ;\{\tau\}) \\
& =\sum_{j \mid m} \frac{1}{j} \sum_{d \mid j} \mu(j / d)\left([m / d<|\tau|] p_{k}\left(d ; \operatorname{merges}_{m / d}(\tau)\right)+[m / d \geq|\tau|] \alpha(d)\right),
\end{aligned}
$$

where $\alpha(d)=\sum_{j=1}^{t-1}\left(\begin{array}{l}k \\ j\end{array}\right)\left\{\begin{array}{l}d \\ j\end{array}\right\}$ is the number of all k-ary $d$-words with fewer than $t$ distinct letters, and $\mu$ is the Moebius function.

Proof. The period of an $m$-word $w$ is the least integer $n$ such that $w=u^{m / n}$ for some word $u$. Fix $m$, and for $j \leq m$, define $f_{j}$ to be the number of $k$-ary $m$-words cyclically avoiding $\tau$ with period dividing $j$. Then by Moebius inversion the number of words with period exactly $j$ is $\sum_{d \mid j} \mu(d) f_{j / d}$, and so the number of all circular words is

$$
\sum_{j \mid m} \frac{1}{j} \sum_{d \mid j} \mu(d) f_{j / d}=\sum_{j \mid m} \frac{1}{j} \sum_{d \mid j} \mu(j / d) f_{d} .
$$

If an $m$-word $w$ has period dividing $d$, then it has the form $w=u^{m / d}$ for a subword $u$ of length $d$. If $m / d \geq|\tau|$ then $w$ cyclically contains $\tau$ if and only if $u$ contains at least as many distinct letters as there are in $\tau$. If $m / d<|\tau|$, then $w$ cyclically avoids 
$\tau$ if and only if $u$ avoids merges $_{m / d}(\tau)$.

\subsection{Note on compositions over $\mathbb{Z}_{k}$}

The problem of counting compositions over a group that avoid a subsequence pattern has not been addressed in prior literature, but was suggested in [GMW18]. Here we present a general technique illustrated for the pattern $1^{\prime}-2-1^{\prime \prime}$.

Proposition 5.10. Let $P_{k}^{\langle a\rangle}(y)$ be the generating function for compositions of a over $\mathbb{Z}_{k}$ avoiding the pattern set $\{1-3-2,2-3-1,1-2-1\}$ (alternatively the partially ordered pattern $\left.1^{\prime}-2-1^{\prime \prime}\right)$, where $y$ marks length. Then

$$
\left[y^{m}\right] P_{k}^{\langle a\rangle}(y)=\frac{1}{k} m^{2 k-2}+O\left(m^{2 k-3}\right), \quad m \rightarrow \infty .
$$

Proof. Let $P_{k}(x, y)$ be the generating function for integer compositions over the part set $[k]$ avoiding $1^{\prime}-2-1^{\prime \prime}$, where $x$ marks total and $y$ marks length. Example 5.62 in [HM10] provides the expression

$$
P_{k}(x, y)=\frac{1}{\prod_{d=1}^{k}\left(1-x^{d} y\right)^{2}}-\sum_{d=1}^{k} \frac{x^{d} y}{\prod_{b=d}^{k}\left(1-x^{b} y\right)^{2}} .
$$

The multisection formula [GJ04, Ex. 1.1.9] for power series $F(z)=\sum_{n} f_{n} z^{n}$ is

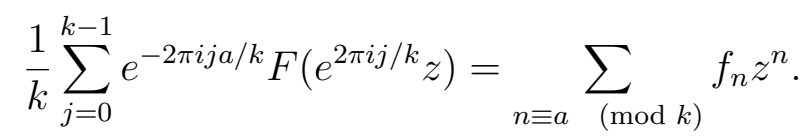




\begin{tabular}{|rr|rrrrrrrrrrr|}
\hline \multicolumn{2}{r|}{$r^{m}$} & 0 & 1 & 2 & 3 & 4 & 5 & 6 & 7 & 8 & 9 & 10 \\
\hline 0 & 1 & 1 & 4 & 12 & 32 & 71 & 150 & 287 & 517 & 877 & 1436 \\
1 & 0 & 1 & 4 & 13 & 34 & 76 & 154 & 294 & 526 & 893 & 1450 \\
2 & 0 & 1 & 4 & 12 & 32 & 74 & 152 & 288 & 518 & 883 & 1440 \\
3 & 0 & 1 & 4 & 13 & 32 & 75 & 154 & 294 & 522 & 891 & 1450 \\
\hline
\end{tabular}

Table 10: Counts of $m$-compositions of $a$ over $\mathbb{Z}_{4}$ avoiding $1^{\prime}-2-1^{\prime \prime}$.

Using the multisection formula we have

$$
\begin{aligned}
P_{k}^{\langle a\rangle}(y) & =\frac{1}{k} \sum_{c=1}^{k} e^{-2 \pi i c a / k} P_{k}\left(e^{2 \pi i c / k}, y\right) \\
& =\frac{1}{k} \sum_{c=1}^{k} e^{-2 \pi i c a / k}\left(\frac{1}{\prod_{d=1}^{k}\left(1-e^{2 \pi i c d / k} y\right)^{2}}-\sum_{d=1}^{k} \frac{e^{2 \pi i c d / k} y}{\prod_{b=d}^{k}\left(1-e^{2 \pi i c b / k} y\right)^{2}}\right)
\end{aligned}
$$

Thus $P_{k}^{\langle a\rangle}(y)$ is rational. We claim that any pole of $P_{k}^{\langle a\rangle}(y)$ other than $y=1$ has order at most $2(k-1)$. All poles other than $y=1$ would come from terms where $c \neq k$. If $c \neq k$, then for $d=1, \ldots, k$, the factor $e^{2 \pi i c d / k}$ takes on at least 2 different values e.g. at $d=1$ and $d=k$, so we conclude the claim. We claim the pole at $y=1$ has order $2 k-1$. Such poles can only come from the term $c=k$. This term is

$$
\begin{aligned}
& \frac{1}{\prod_{d=1}^{k}(1-y)^{2}}-\sum_{d=1}^{k} \frac{y}{\prod_{b=d}^{k}(1-y)^{2}} \\
= & \frac{1}{\prod_{d=1}^{k}(1-y)^{2}}-\frac{y}{\prod_{d=1}^{k}(1-y)^{2}}-\sum_{d=2}^{k} \frac{y}{\prod_{b=d}^{k}(1-y)^{2}} \\
= & \frac{1}{(1-y)^{2 k-1}}+O\left((1-y)^{-2(k-1)}\right) .
\end{aligned}
$$

We conclude the second claim and the proposition follows by applying Theorem 2.2.

Table 10 shows initial coefficients of $P_{4}^{\langle a\rangle}(y)$.

Similar analysis can potentially be performed e.g. for compositions avoiding a length3 permutation pattern as enumerated in [HM10, Theorem 5.7] and those avoiding the 
pattern 1-1-2 as enumerated in Theorem 5.13 in [HM10, p. 139].

Remark 5.4. Note that if we wanted to count compositions mod $k$ using a recurrence relation that recurses on $k$, we have the following problem. While we can create a composition over $[k]$ by creating one over $[k-1]$ and inserting some copies of $k$, we must know the total of the composition over $[k-1]$ as a value $\bmod k$, not $\bmod k-1$. So the alphabet and modulus have to be tracked separately. 


\section{Conclusion}

As a conclusion, this section mentions some relevant problems which are as yet unsolved. The book [HM10] contains a variety of proposed research problems many of which are also unsolved.

The paper [BM05] uses finite automata to count words avoiding a subsequence pattern. The only cycles in the automata are loops and so an asymptotic form for the number of accepted words is obtained directly. Rather than using the technique of $\$ 5.2$, it may be possible to count compositions over a finite group that avoid a subsequence pattern by combining the techniques of [BM05] and $\S 2.1$.

Consider locally restricted compositions where the parts come from a finite generating set of an infinite group. In the framework of $\S 2.1$, the base digraph $D$ is finite but the derived digraph $D_{\times}$is infinite. Take for example the infinite group $\mathbb{Z}$ with generating set $\{-1,0,1\}$. Unrestricted compositions over $\{-1,0,1\}$ with total, say, 0 no longer form a regular language but do form a context-free language, recognizable by a pushdown automaton. There may be difficulty from finitely generated non-abelian groups, however, due to the fact that the word problem is undecidable and therefore not even context-free. For finitely-generated abelian groups, it is possible that the number of locally restricted compositions of $a$ is asymptotically independent of $a$ but it is no longer possible for each total $a$ to be asymptotically equally likely since the group is infinite. The recent paper [DFW18] explores other problems involving finitely-generated groups and enumeration.

Suppose we have a group $G$ which is infinite but we also have a weight function $W: G \rightarrow \mathbb{Z}_{>0}$. As long as each preimage $W^{-1}(n)$ is finite, we can define the number 
of locally restricted compositions of $a$ over $G$ with a given total weight $n$. It is plausible that approaches in the above sections and [BC09] can be applied to this counting problem.

Circular integer compositions (unlabeled cycles weighted by positive integers) appeared in the early paper [SY95]. More recently, the enumerative study of locally restricted circular integer compositions has progressed in [Had16; Had17] which study Carlitz compositions and restrictions on the set of parts. The conclusion section of [Had17] suggests expanding to general local restrictions as in [BC09]. Other recent work [GJW18] has looked at part sizes in circular integer compositions.

We can also consider a set of colored parts $(j, c)$, where $j \in \mathbb{Z}_{>0}$ and $c$ comes from a set of colors (with no particular algebraic structure). A colored integer composition of $n$ is a sequence $\left(\left(j_{1}, c_{1}\right), \ldots,\left(j_{m}, c_{m}\right)\right)$ where $\sum_{i} j_{i}=n$. Questions about restricted integer compositions can be asked again for colored integer compositions and for colored versions of compositions over a finite group. Some results and open problems mentioned in [BG18] are relevant.

In the sections above on local restrictions, we focus on "implicit" results that cover a wide range of particular restrictions. What this does not provide is a "simple" formula for exact counts, or explicit constants within asymptotic expressions. So there is the possibility of finding (more) explicit, but less general, expressions to complement our implicit ones. For a deep discussion of the meaning of explicitness in enumeration, see [Wil82; Pak18].

In $\S 5$ we count $k$-ary words of length $m$ avoiding a subsequence pattern set $T$. A further parameter can be tracked, namely the length $p$ of the longest contiguous run of a single letter. This problem for subword pattern avoidance is addressed in 
[BG16] but there is no previous work for subsequence patterns. It is quickly deduced that this is roughly equivalent to counting words that avoid $T$ and also avoid the subword pattern $1^{p}$. Combining subsequence and subword pattern avoidance presents a challenge.

Finite mappings from a set to itself correspond to functional digraphs, which have a well-known structure [FO89]. Research such as [AB82] has enumerated functional digraphs with a kind of local restriction: the indegree of each vertex (a.k.a. the number of its preimages) must lie within a fixed set $\Xi$. More recent papers such as $[$ Mar+17] consider the distribution of the least common multiple $T$ and product $B$ of the cycle lengths in restricted functional digraphs, for particular $\Xi$. These values $T, B$ are related to the sequence of iterations of the mapping. Problems remain such as expanding to more general $\Xi$. 


\section{References}

[Alb+01] M. H. Albert, R. E. Aldred, M. D. Atkinson, C. Handley, and D. Holton. "Permutations of a multiset avoiding permutations of length 3". In: European Journal of Combinatorics 22.8 (2001), pp. 1021-1031.

[AB82] J. Arney and E. Bender. "Random mappings with constraints on coalescence and number of origins". In: Pacific Journal of Mathematics 103.2 (1982), pp. 269-294.

[ALW95] M. Atkinson, S. Linton, and L. Walker. "Priority queues and multisets". In: the Electronic Journal of Combinatorics 2.1 (1995), R24.

[BC09] E. A. Bender and E. R. Canfield. "Locally restricted compositions II. General restrictions and infinite matrices". In: the Electronic Journal of Combinatorics 16.1 (2009), R108.

[BCG12] E. A. Bender, R. Canfield, and Z. Gao. "Locally Restricted Compositions IV. Nearly Free Large Parts and Gap-Freeness". In: Discrete Mathematics E3 Theoretical Computer Science (2012).

[BG14] E. A. Bender and Z. Gao. "Part sizes of smooth supercritical compositional structures". In: Combinatorics, Probability and Computing 23.5 (2014), pp. 686-716.

[BG16] E. A. Bender and Z. Gao. "Locally restricted sequential structures and runs of a subcomposition in integer compositions". In: arXiv preprint arXiv:1605.04353 (2016). 
[BG18] E. A. Bender and Z. Gao. "Locally restricted sequential structures and runs of a given subcomposition in random locally restricted integer compositions". In preparation. 2018.

[BG75] E. A. Bender and J. R. Goldman. "On the applications of Mobius inversion in combinatorial analysis". In: American Mathematical Monthly (1975), pp. 789-803.

[BRW83] E. A. Bender, L. B. Richmond, and S. Williamson. "Central and local limit theorems applied to asymptotic enumeration. III. Matrix recursions". In: Journal of Combinatorial Theory, Series A 35.3 (1983), pp. 263-278.

[Ber+03] A. Bertoni, C. Choffrut, M. Goldwurm, and V. Lonati. "On the number of occurrences of a symbol in words of regular languages". In: Theoretical Computer Science 302.1-3 (2003), pp. 431-456.

[Bil08] P. Billingsley. Probability and Measure. John Wiley \& Sons, 2008.

[BM76] J. A. Bondy and U. S. R. Murty. Graph Theory with Applications. Vol. 290. North-Holland, 1976.

[BM05] P. Brändén and T. Mansour. "Finite automata and pattern avoidance in words". In: J. Comb. Theory Ser. A 110.1 (2005), pp. 127-145.

[Bur98] A. Burstein. "Enumeration of words with forbidden patterns". PhD thesis. University of Pennsylvania, 1998.

[BK08] A. Burstein and S. Kitaev. "Partially ordered patterns and their combinatorial interpretations". In: Pure Math. and Appl. (PU.M.A.) 19.2-3 (2008), pp. 27-38.

[BM02] A. Burstein and T. Mansour. "Words restricted by patterns with at most 2 distinct letters". In: The Electronic Journal of Combinatorics 9.2 (2002). 
[BM03] A. Burstein and T. Mansour. "Counting occurrences of some subword patterns". In: Discrete Math. Theor. Comput. Sci. 6.1 (2003), pp. 1-11.

[Chy+08] F. Chyzak, M. Drmota, T. Klausner, and G. Kok. "The distribution of patterns in random trees". In: Combinatorics, Probability and Computing 17.1 (2008), pp. 21-59.

[DFW18] F. Dahmani, D. Futer, and D. T. Wise. "Growth of quasiconvex subgroups". In: Mathematical Proceedings of the Cambridge Philosophical Society. Cambridge University Press. 2018, pp. 1-26.

[Dai+12] M. Dairyko, L. Pudwell, S. Tyner, and C. Wynn. "Non-Contiguous Pattern Avoidance in Binary Trees". In: The Electronic Journal of Combinatorics 19.3 (2012), P22.

[Drm09] M. Drmota. Random Trees: An Interplay Between Combinatorics and Probability. Springer Science \& Business Media, 2009.

[FM09] G. Firro and T. Mansour. "Restricted $k$-ary words and functional equations". In: Discrete Appl. Math. 157.4 (2009), pp. 602-616.

[FO89] P. Flajolet and A. M. Odlyzko. "Random mapping statistics". In: Workshop on the Theory and Application of of Cryptographic Techniques. Springer. 1989, pp. 329-354.

[FS09] P. Flajolet and R. Sedgewick. Analytic Combinatorics. Cambridge University Press, Cambridge, 2009.

[Gab+12] N. Gabriel, K. Peske, L. Pudwell, and S. Tay. "Pattern avoidance in ternary trees". In: Journal of Integer Sequences 15.2 (2012), p. 3. 
[GMP11] Z. Gao, A. MacFie, and D. Panario. "Counting words by number of occurrences of some patterns". In: The Electronic Journal of Combinatorics 18.1 (2011), P143.

[GMW18] Z. Gao, A. MacFie, and Q. Wang. "Counting compositions over finite abelian groups". In: The Electronic Journal of Combinatorics 25.2 (2018), P2.19.

[GJW18] M. M. Gibson, M. Just, and H. Wang. "Note on restricted parts in cyclic compositions". In: INTEGERS 18 (2018), p. 2.

[GJ04] I. P. Goulden and D. M. Jackson. Combinatorial Enumeration. Courier Corporation, 2004.

[GKP94] R. L. Graham, D. E. Knuth, and O. Patashnik. Concrete Mathematics. Second edition. A foundation for computer science. Addison-Wesley Publishing Company, Reading, MA, 1994, pp. xiv+657.

[GT87] J. L. Gross and T. W. Tucker. Topological Graph Theory. Dover, 1987.

[Gut05] A. Gut. Probability: A Graduate Course. Springer-Verlag, 2005.

[Had16] P. Hadjicostas. "Cyclic compositions of a positive integer with parts avoiding an arithmetic sequence". In: Journal of Integer Sequences 19.2 (2016), p. 3 .

[Had17] P. Hadjicostas. "Cyclic, Dihedral and Symmetrical Carlitz Compositions of a Positive Integer". In: Journal of Integer Sequences 20.2 (2017), p. 3.

[HKM07] S. Heubach, S. Kitaev, and T. Mansour. "Partially ordered patterns and compositions". In: Pure Math. and Appl. (PU.M.A.) 17.1-2 (2007), pp. 112. 
[HM06] S. Heubach and T. Mansour. "Avoiding patterns of length three in compositions and multiset permutations". In: Advances in Applied Mathematics 36.2 (2006), pp. 156-174.

[HM10] S. Heubach and T. Mansour. Combinatorics of Compositions and Words. Chapman \& Hall/CRC, 2010.

[HJ94] R. A. Horn and C. R. Johnson. Topics in Matrix Analysis. Corrected reprint of the 1991 original. Cambridge University Press, Cambridge, 1994.

[JM09] V. Jelnek and T. Mansour. "Wilf-equivalence on k-ary words, compositions, and parking functions". In: The Electronic Journal of Combinatorics 16.R58 (2009), p. 1.

[Kit05] S. Kitaev. "Segmented partially ordered generalized patterns". In: Theor. Comp. Sci. 349.3 (2005), pp. 420-428.

[KM03] S. Kitaev and T. Mansour. "Partially Ordered Generalized Patterns and k-ary Words". In: Ann. Combin. 7.2 (2003).

[KMM11] A. Knopfmacher, T. Mansour, and A. Munagi. "Smooth compositions and smooth words". In: Pure Math. and Appl. (PU.M.A.) 22.2 (2011), pp. 209-226.

[Kno+10] A. Knopfmacher, T. Mansour, A. Munagi, and H. Prodinger. "Staircase words and Chebyshev polynomials". In: Applicable Analysis and Discrete Mathematics (2010), pp. 81-95.

[Knu92] D. E. Knuth. "Two notes on notation". In: The American Mathematical Monthly 99.5 (1992), pp. 403-422.

[Mac14] A. MacFie. Genfunlib. Apr. 2014. 
[Mac16] A. MacFie. "Software for enumerative and analytic combinatorics". In: abs/1601.02683 (2016). arXiv: 1601.02683.

[ML10] N. Madras and H. Liu. "Random pattern-avoiding permutations". In: Algorithmic Probability and Combinatorics, AMS, Providence, RI (2010), pp. 173-194.

[Man06] T. Mansour. "Restricted 132-avoiding $k$-ary words, Chebyshev polynomials, and continued fractions". In: Adv. in Appl. Math. 36.2 (2006), pp. 175193.

[MS15] T. Mansour and M. Shattuck. "On avoidance of patterns of the form $\sigma-\tau$ by words over a finite alphabet". In: Discrete Mathematics 83 Theoretical Computer Science Vol. 17 no.2 (Sept. 2015).

[MS06] T. Mansour and B. Sirhan. "Counting $l$-letter subwords in compositions". In: Discrete Mathematics and Theoretical Computer Science 8 (2006), pp. 285-297.

[Mar+17] R. S. Martins, D. Panario, C. Qureshi, and E. Schmutz. "Periods of iterations of mappings over finite fields with restricted preimage sizes". In: arXiv preprint arXiv:1701.09148 (2017).

[Mey00] C. D. Meyer. Matrix Analysis and Applied Linear Algebra. Vol. 71. Siam, 2000.

$[$ Olv +17$] \quad$ F. W. J. Olver, A. B. Olde Daalhuis, D. W. Lozier, B. I. Schneider, R. F. Boisvert, C. W. Clark, B. R. Miller, and B. V. Saunders. NIST Digital Library of Mathematical Functions. Release 1.0.15. 2017.

[Pak18] I. Pak. "Complexity problems in enumerative combinatorics". In: abs/1803.06636 (2018). arXiv: 1803.06636. 
[Pud08] L. Pudwell. "Enumeration schemes for pattern-avoiding words and permutations". PhD thesis. Rutgers, The State University of New Jersey, 2008.

[RE04] B. Ravikumar and G. Eisman. "Weak minimization of DFA-an algorithm and applications". In: Theoretical computer science 328.1-2 (2004), pp. 113-133.

[Reg98] A. Regev. "Asymptotics of the number of $k$-words with an $l$-descent". In: The Electronic Journal of Combinatorics 5 (1998), R15.

[Res13] S. I. Resnick. A Probability Path. Springer Science \& Business Media, 2013.

[Row10] E. S. Rowland. "Pattern avoidance in binary trees". In: Journal of Combinatorial Theory, Series A 117.6 (2010), pp. 741-758.

[SW06] C. D. Savage and H. S. Wilf. "Pattern avoidance in compositions and multiset permutations". In: Advances in Applied Mathematics 36.2 (2006), pp. 194-201.

[Sch74] H. H. Schaefer. Banach Lattices and Positive Operators. Die Grundlehren der mathematischen Wissenschaften, Band 215. Springer-Verlag, New York-Heidelberg, 1974, pp. xi+376.

[Sch17] M. D. Schmidt. "Square series generating function transformations". In: Journal of Inequalities and Special Functions 8.2 (2017), pp. 125-156.

[SY95] R. Servedio and Y.-N. Yeh. "A bijective proof on circular compositions". In: Bulletin of the Institute of Mathematics Academia Sinica 23 (1995), pp. $283-294$. 
[Sha08] J. Shallit. A Second Course in Formal Languages and Automata Theory. Cambridge University Press, 2008.

[Sta07] R. P. Stanley. "Increasing and decreasing subsequences and their variants". In: International Congress of Mathematicians. Vol. 1. 2007, pp. 545-579.

[Sta12] R. P. Stanley. Enumerative Combinatorics. Second edition. Vol. 1. Cambridge Studies in Advanced Mathematics. Cambridge University Press, Cambridge, 2012.

[SH85] R. E. Stearns and H. B. Hunt III. "On the equivalence and containment problems for unambiguous regular expressions, regular grammars and finite automata". In: SIAM Journal on Computing 14.3 (1985), pp. 598611.

[Wil82] H. Wilf. "What is an answer?" In: J. Amer. Math. Monthly 89.5 (1982), pp. 289-292.

[Zan17] R. Zanasi. System and control theory. University of Modena and Reggio Emilia. 2017.

[Zei00] D. Zeilberger. "The umbral transfer-matrix method. I. Foundations". In: Journal of Combinatorial Theory, Series A 91.1-2 (2000), pp. 451-463. 